\title{
Studying Media Access and Control Protocols
}

ALALELDDIN MOHAMMED

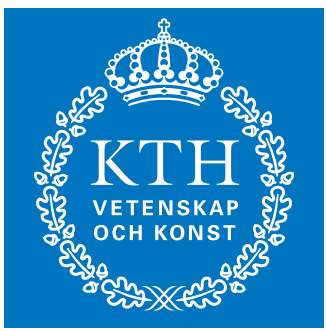

KTH Information and Communication Technology 


\title{
Studying Media Access and Control Protocols
}

\author{
Alalelddin Mohammed \\ Email: hdm06amo@sth.kth.se
}

Master of Science Thesis

Date: 2010-01-19

Supervisor and Examiner

Prof. Gerald Q. Maguire Jr. 


\section{Abstract}

This thesis project's goal is to enable undergraduate students to gain insight into media access and control protocols based upon carrying out laboratory experiments. The educational goal is to de-mystifying radio and other link and physical layer communication technologies as the students can follow packets from the higher layers down through the physical layer and back up again.

The thesis fills the gap between the existing documentation for the Universal Software Radio Peripheral (USRP) resources and the knowledge of undergraduate students. This was necessary because the existing document is targeted at advanced audiences rather than undergraduates. This thesis describes the design and evolution of a workbench for students to experiment with a variety of media access and control protocols, much as Wireshark gives students the ability to watch network and higher layer protocols. Another motivation for this thesis is that an increasing number of communication networks use complex media access and control protocols and existing tools do not allow students to see the details of what is taking place in these protocols, except via simulation. Today an software defined radio and computer are affordable as laboratory equipment for an undergraduate course. Hence the time is ripe for the development of undergraduate laboratory course material using these tools.

The thesis is targeted at (1) instructors of undergraduates who might use this work to develop their own lesson plans and course material and (2) students of physical and link layer protocols who want a practical tool for carrying out experiments in these layers. Hopefully by de-mystifying these lower layers and by making the USRP more approachable by undergraduate students we will encourage lots of students to view wireless network technology as being just as approachable as a wired Ethernet.

Due to the widespread use of wireless communications technologies, there is a great need by industry for more graduates who can understand communication systems from the physical to the application layer - rather than the current situation where there is a hard boundary between the lower two layers and the upper layers. While there has been a lot of research concerning cross layer optimization, much of this is theoretical and not very approachable by students. A desired outcome of this thesis project is that undergraduate students will be able to understand tradeoffs at all layers of the protocol stack and not be limited to the upper layers. 


\section{Sammanfattning}

Detta examensarbete har som mål att göra det möjligt för studenter att få inblick i tillgång till medierna och protokoll som grundar sig på att utföra laboratorieexperiment. Det pedagogiska målet är att de-mystifierande radio och annan länk och fysiska lagret kommunikationsteknik som studenterna kan följa paket från högre skikt ner genom det fysiska lagret och upp igen.

Avhandlingen fyller gapet mellan den befintliga dokumentationen för Universal Software Radio Peripheral (usrp) resurser och kunskap om studerande. Detta var nödvändigt eftersom det befintliga dokument riktar sig till avancerade publik snarare än studenter. Denna avhandling beskriver utformningen och utvecklingen av en arbetsbänk för studenter att experimentera med olika tillgång till medierna och protokoll kontroll, mycket som Wireshark ger studenterna möjlighet att titta på nätet och högre skikt protokoll. Ett annat motiv för denna tes är att ett ökande antal kommunikationsnät använda komplicerade tillgång till medierna och protokoll kontroll och befintliga verktyg inte tillåter eleverna att se detaljer om vad som sker i dessa protokoll, utom via simulering. Idag en programvarustyrd radio och dator är överkomliga laboratorieutrustning för en grundutbildningskurs. Därför är tiden mogen för utvecklingen av grundutbildningen laborationer material med hjälp av dessa verktyg.

Avhandlingen riktar sig till (1) instruktörer för studenter som kan använda detta arbete för att utveckla sin egen lektionsplanering och kursmaterial och (2) studenter på fysisk och länka protokoll skikt som vill ha ett praktiskt verktyg för att utföra experiment i dessa lager. Förhoppningsvis genom de-mystifierande de undre lagren och genom att göra usrp mer tillgänglig genom att studenter ska vi uppmuntra många elever att visa trådlös nätverksteknik vara lika lättillgänglig som ett ethernet.

På grund av den utbredda användningen av trådlös kommunikationsteknik, finns ett stort behov från näringslivet för fler studenter som kan förstå kommunikationssystem från det fysiska till applikationslagret - i stället för den nuvarande situationen där det finns en hård gräns mellan de två lägre skikten och de övre skikten. Samtidigt som det har varit en hel del forskning om cross lager optimering, mycket av detta är teoretisk och inte särskilt tillgänglig av studenter. Ett önskat resultat med detta examensarbete är att studenter ska kunna förstå kompromisser på alla nivåer inom den protokollstack och inte vara begränsade till de övre skikten. 


\section{Acknowledgement}

Let us think! We always look on the bright side of life .... From him I learn Knowledge not in the books! What to think and how to look for the best solution! When I deliver my problems to him, the feed6ack was always new knowledge acquired to make this work possible. Chip Maguire my supervisor Thank you.... we swim in your Knowledge. 


\section{Table of Contents}

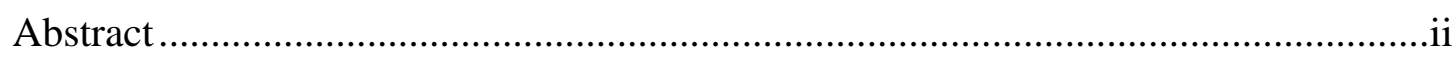

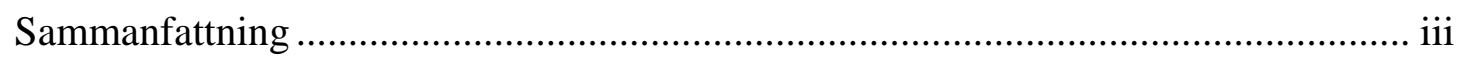

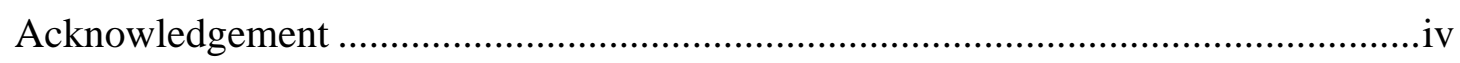

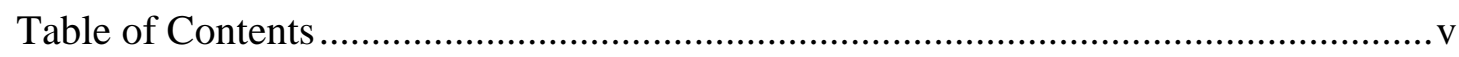

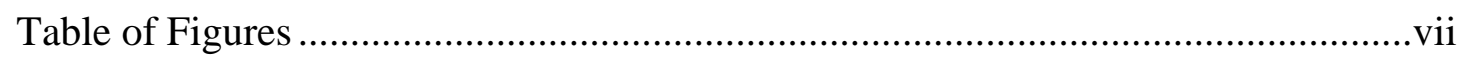

List of Code Examples..................................................................................... viii

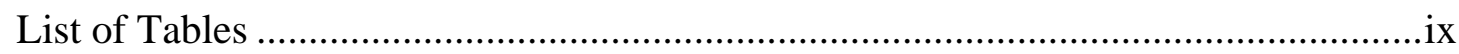

List of Acronyms and Abbreviations.......................................................................

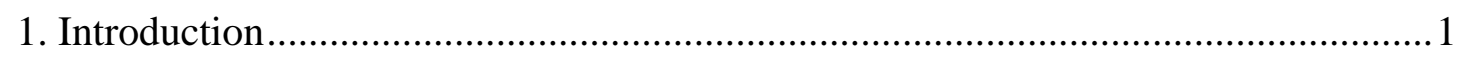

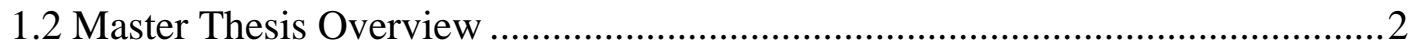

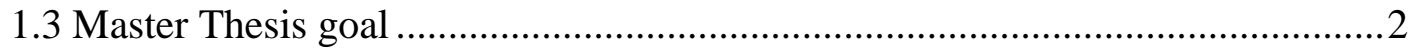

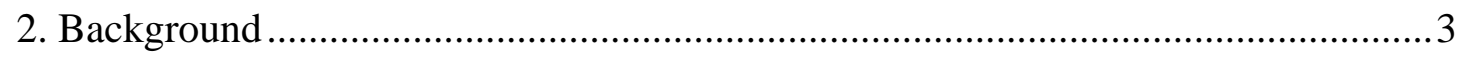

2.1 Software Defined Radio (SDR) History ........................................................

2.2 Modern Software Defined Radio ..................................................................4

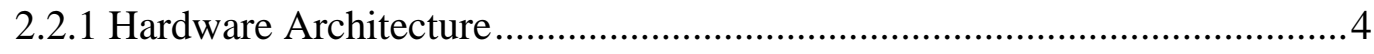

2.3 Software Defined radio (SDR) and Software Radio (SR) ................................

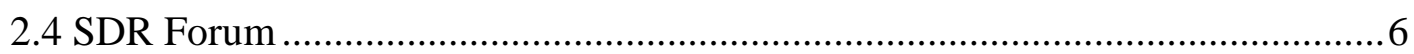

3. The Universal Software Radio Peripheral (USRP) ...............................................

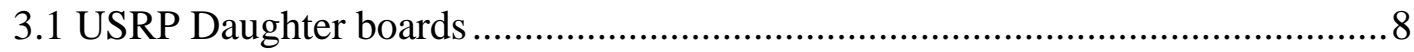

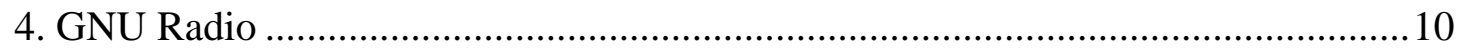

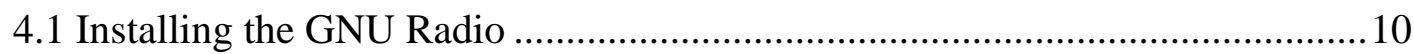

4.1.1 Installing GNU Radio on Fedora 10 ......................................................11

4.1.2 Installing GNU Radio on Ubuntu 8.04 ................................................12

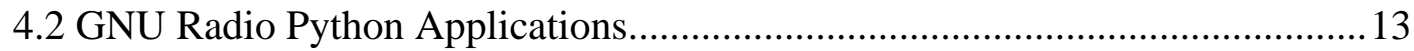

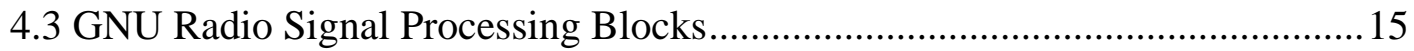

4.3.1 Creating a Simple Signal Processing Block..............................................18

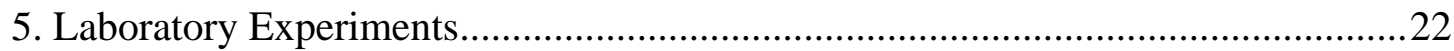

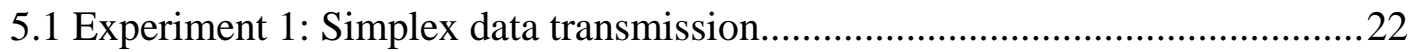

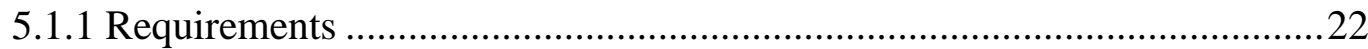

5.1.2 Simplex data transmission implementation ............................................22

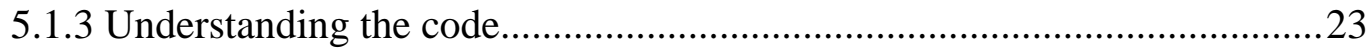

5.1.4 Setup and Perform a Simplex Data transmission........................................225

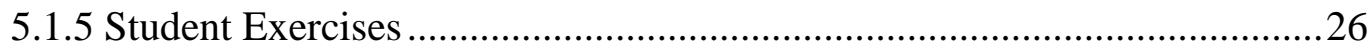




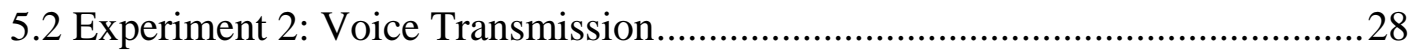

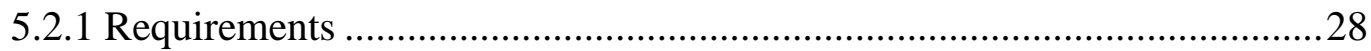

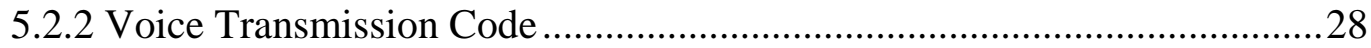

5.2.3 Setup and Run Voice Transmission .......................................................28

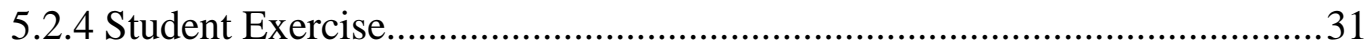

5.3 Experiment 3: Carrier Sense Multiple Access Protocol ...................................32

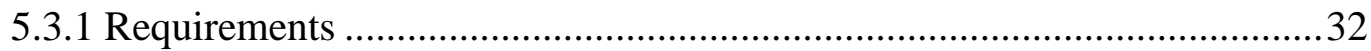

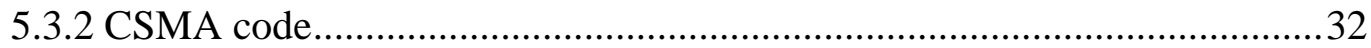

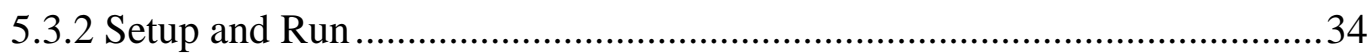

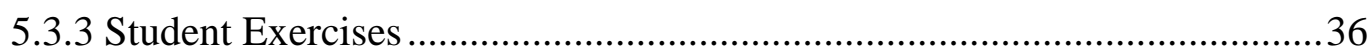

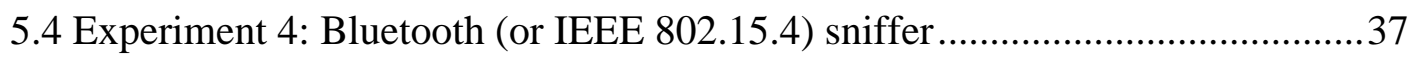

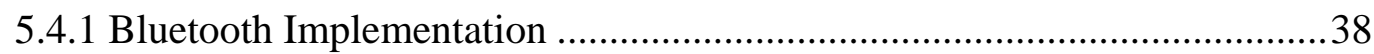

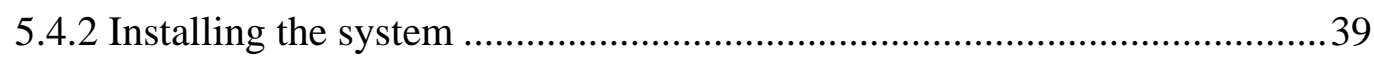

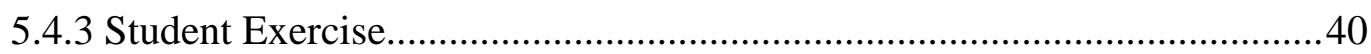

5.5 Experiment 5: IEEE 802.11 Implementation...................................................43

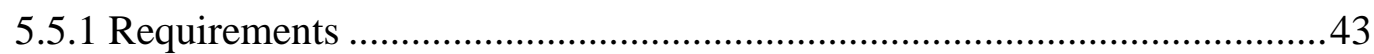

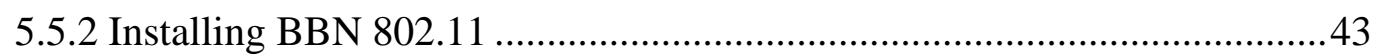

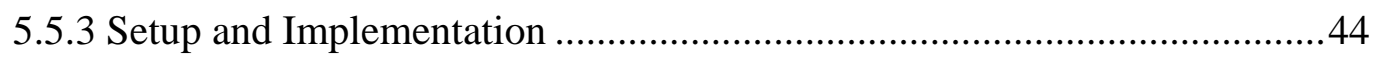

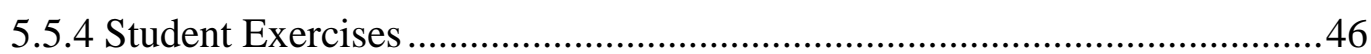

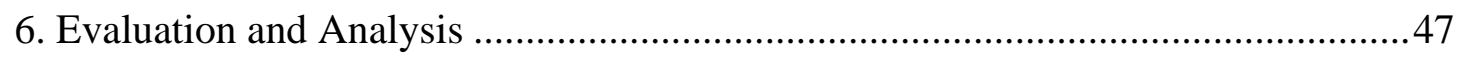

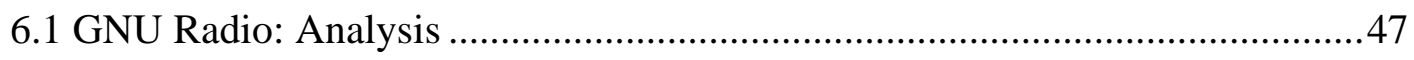

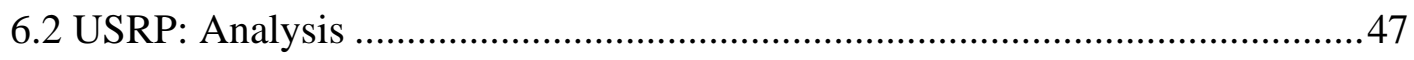

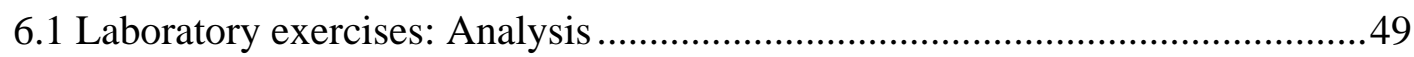

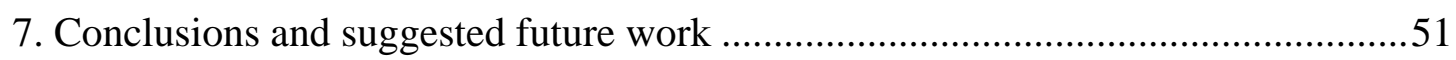

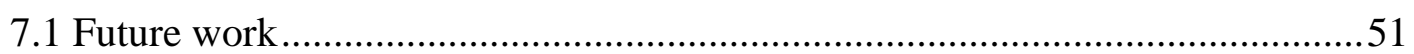

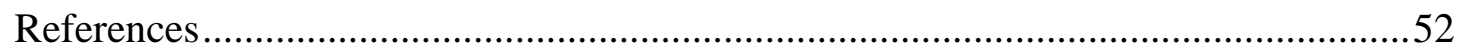

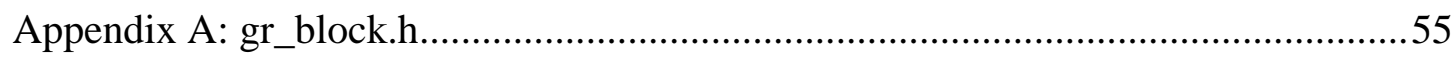

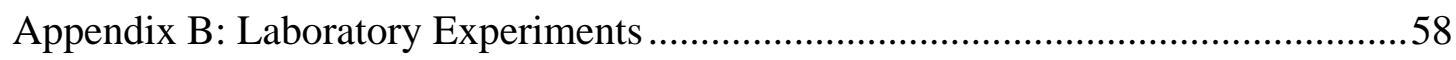

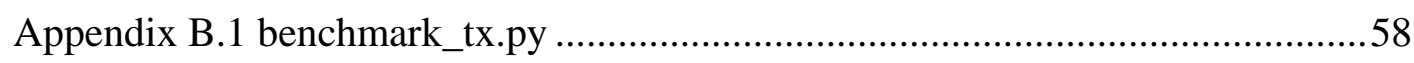

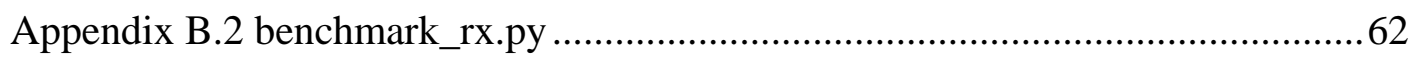

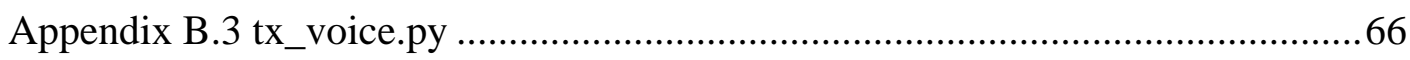

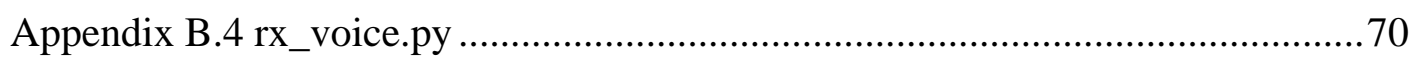

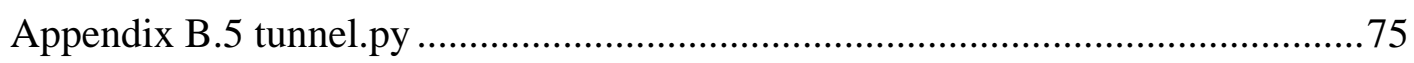




\section{Table of Figures}

Figure 1: Introduction to USRP and GNU Radio ..................................................

Figure 2: Basic hardware architecture of a modern SDR. .........................................4

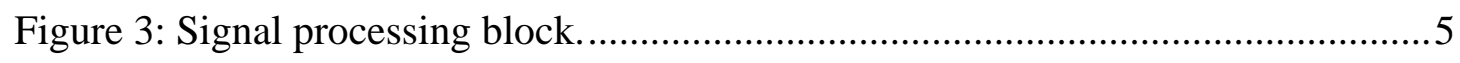

Figure 4: Universal Software Radio Peripheral (USRP) ...........................................

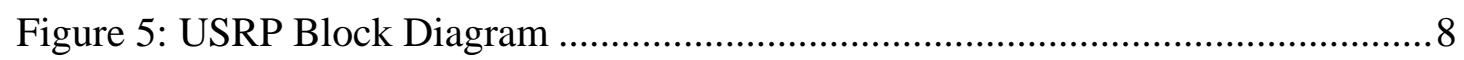

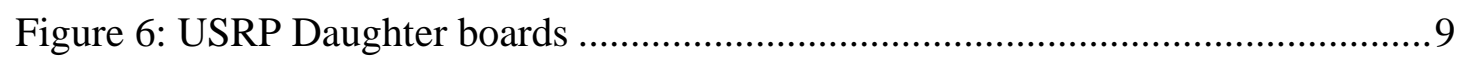

Figure 7: A basic SDR system based on GNU Radio and USRP ...............................10

Figure 8: Adding Repositories Using Software Sources in Ubuntu 8.04 ....................12

Figure 9: Flow Graph to generate a Dial Tone ........................................................... 14

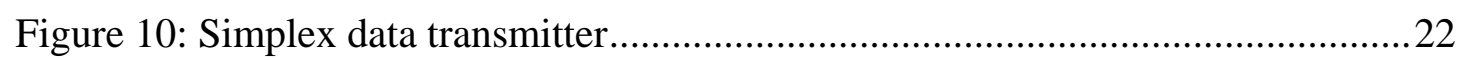

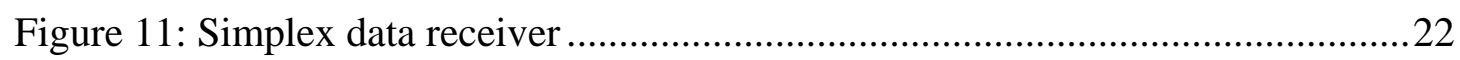

Figure 12: Simplex data transmission; showing the relation between the packet and

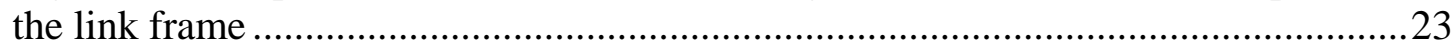

Figure 13: setup USRP for loopback simplex communication....................................25

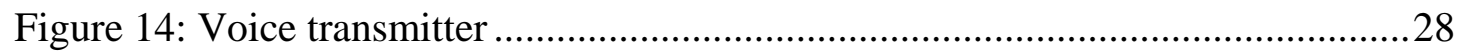

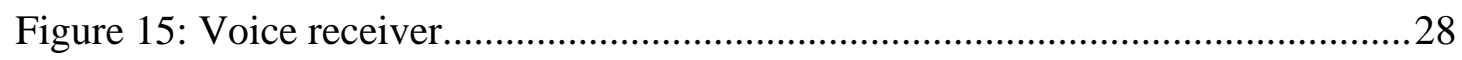

Figure 16: Connecting USRPA Basic RX $\rightarrow$ USRPB Basic TX ..............................29

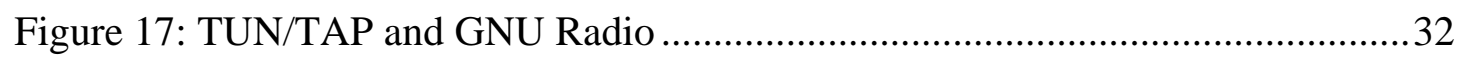

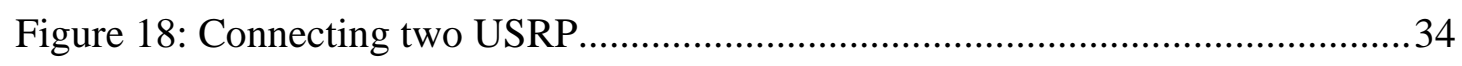

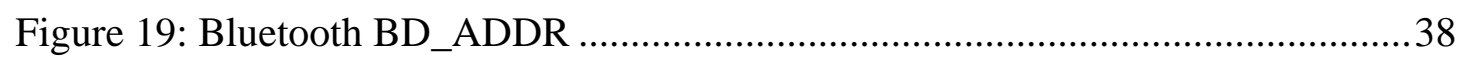

Figure 20: USRP $2.4 \mathrm{GHz}$ Antenna (designed for use with WLAN devices).............40

Figure 21: RFX2400, with an Antenna. Note the two sides of the USRP (A and B)...44 


\section{List of Code Examples}

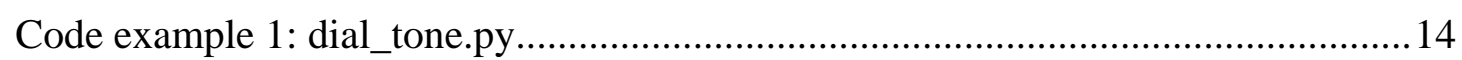

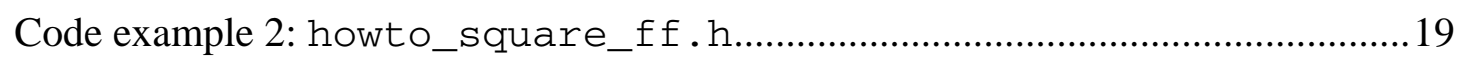

Code example 3: howto_square_ff.CC.........................................................20

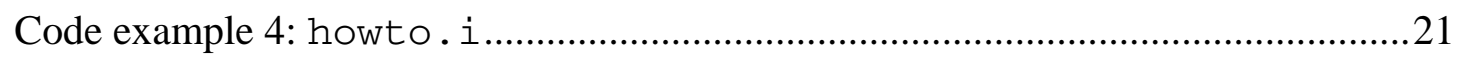

Code example 5: src/lib/Makefile.am .........................................................2 21

Code example 6: benchmark_tx.py; generate and send a packet, sleep after

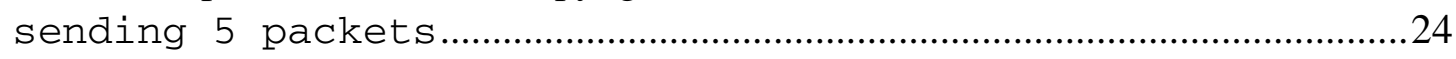

Code example 7: Print packet summary for a receiver packet.....................................25

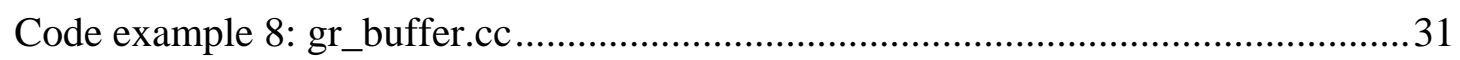

Code example 9: CSMA (transmitter side is is implemented by the main_loop, while the receiver is implemented by the phy_rx_callback) ...................................................33 


\section{List of Tables}

Table 1: SDR time line with some representation examples[3] [16]............................3

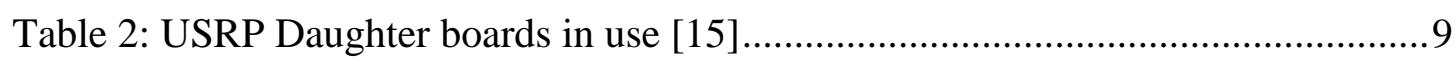

Table 3: GNU Radio Signal Processing Blocks .......................................................17

Table 4: Directory layout of a new signal srocessing block [22] ............................... 18

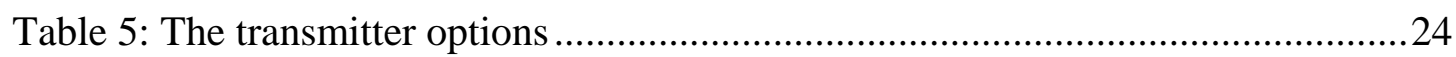

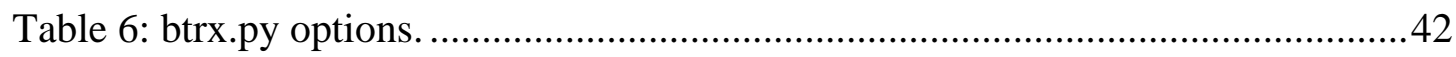

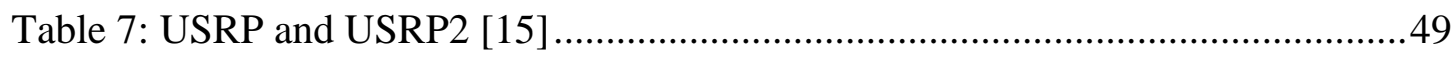




\section{List of Acronyms and Abbreviations}

$\begin{array}{ll}\mathrm{GHz} & \text { Giga Hertz } \\ \mathrm{Hz} & \text { Hertz } \\ \text { MAC } & \text { Media Access and Control } \\ \text { RF } & \text { Radio Frequency } \\ \text { SDR } & \text { Software-Defined Radio } \\ \text { SR } & \text { Software Radio } \\ \text { SWING } & \text { Simplified Wrapper and Interface Generator } \\ \text { USRP } & \text { Universal Software Radio Peripheral }\end{array}$




\section{Introduction}

The idea of a software-defined radio (SDR) is that all the modulation and demodulation is done via software, rather than by specialized circuits. The benefit according to Susan Karlin is "instead of having to build extra circuitry to handle different types of radio signals, you can just load an appropriate program" [1]. An SDR uses programmable digital devices to accomplish the signal processing, instead of fixed hardware.

SDR introduces flexibility and rapid development to radio communication systems by using a software-oriented approach. As software-based approach offers greater flexibility when developing wireless communication systems, since the wireless system architecture is not frozen into the hardware, but can be changed at any time via changing the software which is loaded into the device. By delaying the binding of design decisions until execution time, the designers can incorporate the latest developments - enabling them to improve the performance of the systems. This reduces the difference between the state of the art and the state of practice for wireless communication systems. Additionally, this software-oriented approach to wireless communication devices allows both flexibility and simpler maintenance, as most upgrades can be done by loading new software, rather than changing physical modules.

The Ettus Research Universal Software Radio Peripheral (USRP) is an example of an SDR. It provides an "RF front end for a computer running the GNU Radio software, converting radio waves picked up by an antenna into digital copies that the computer software can handle or, conversely, converting a wave synthesized by the computer into a radio transmission" [1]. This device can also be viewed as a general purpose front end for receiving and generating all sorts of different kinds of signals (see Figure 1). In Figure 1, "IF" standards for intermediate frequency, representing a version of the signal at a lower frequency that the actual RF. Note that the bandwidth of the signal will need to be at least twice the radio frequency bandwidth to avoid aliasing (As per Shannon's sampling theorem.)

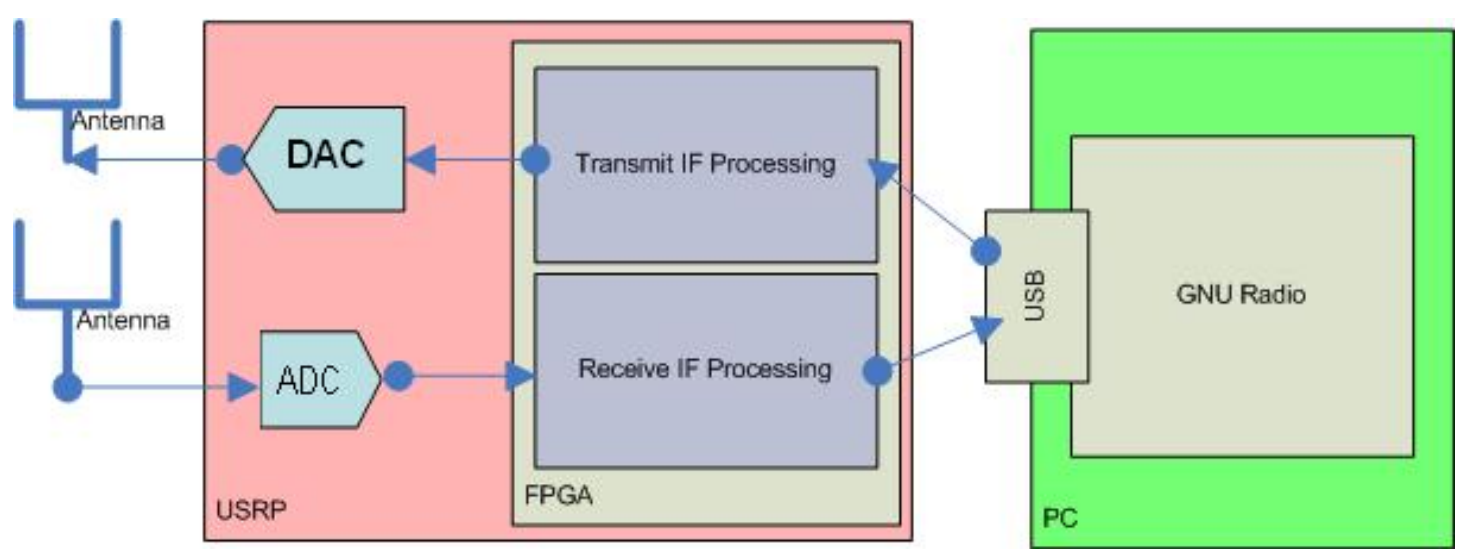

Figure 1: Introduction to USRP and GNU Radio

The USRP motherboard can have up to two transmitters and two receivers that can simultaneously transmit and receive from antennas (or wired connections) in real time. There are various types of daughterboards that can be plugged into the USRP motherboard to provide an interface between the baseband signal and a number of different frequency ranges. USRP was design to operate in a number of different portions of the spectrum ranging from 0 $\mathrm{Hz}$ to $2.9 \mathrm{GHz}$. This wide range covers a large variety of different applications. In this thesis 
we will take advantage of this hardware platform to enable students to both observe and create a number of different media access and control protocols.

\subsection{Master Thesis Overview}

This master's thesis will design, implement, and evaluate a number of lab exercises for undergraduate students using the USRP technology to understand a number of different media access and control (MAC) protocols. Lab exercises will explore different types of signals and MAC protocols. This thesis contains this introduction, followed by chapter 2 that provides some basic background information concerning SDR. Chapter 3 describes the particular SDR hardware platform that we have chosen (i.e., the USRP). Chapter 4 describes the GNU Radio software that we have built upon. Chapter 5 describes some of the laboratory exercises that have been designed during this project. Chapter 6 evaluates these laboratory exercises from a pedagogical point of view. While Chapter 7 presents our conclusions and suggests some future work. A number of appendices are included, containing the complete laboratory exercises; along with details for the student (or instructor) on how to set up a suitable laboratory environment.

\subsection{Master Thesis goal}

This thesis project has two goals:

1. Show a software defined radio application built on USRP and GNU Radio.

2. Develop laboratory exercises for undergraduate students, using USRP and GNU Radio to explain the physical and MAC layers using examples drawn from popular networks that the students are likely to encounter. These exercises cover different applications with both wired network technology and several wireless communication technologies. 


\section{Background}

This chapter introduces software defined radio - beginning with some of its history, moving on to a discussion of underlying hardware architecture of an SDR, and describing the role of the SDR forum in the development of SDR.

\subsection{Software Defined Radio (SDR) History}

A SDR is a radio in which software defines signals, frequencies, modulation, and (optionally) cryptography. SDR design began 1987, when the United States Air Force's Rome Laboratory (AFRL) developed a programmable modem. The modem was based on the Integrated Communications, Navigation, and Identification Architecture (ICNIA) [3]. Despite of this earlier effort, Walter Tuttlebee argues that "Until the mid-1990's most readers would probably not have even come across the term SDR". The term software defined radio was introduced by Joseph Mitola III in 1991 "to signal the shift from digital radio to multiband multimode software-defined radios where " $80 \%$ " of the functionality is provided in software, versus the "80\%" hardware of the 1990's." [23]. Table 1 shows the time line of the development of software defined radio.

Table 1: SDR time line with some representation examples[3] [16]

\begin{tabular}{|c|c|c|c|}
\hline Prject & Year & Size & Features \\
\hline ICNIA & 1978 & Fit in a small room & $\begin{array}{l}\text { A collection of several } \\
\text { single-purpose radios in } \\
\text { one box }\end{array}$ \\
\hline Speakeasy Phase I & 1992 & Six foot $(182 \mathrm{~cm})$ rack & $\begin{array}{l}\text { Included a programmable } \\
\text { cryptography chip. }\end{array}$ \\
\hline Speakeasy Phase II & 1995 & $\begin{array}{l}\text { Stack of two pizza } \\
\text { boxes }\end{array}$ & $\begin{array}{l}\text { The first SDR to include } \\
\text { a voice coder and digital } \\
\text { signal processing } \\
\text { resources. }\end{array}$ \\
\hline Digital Modular Radio & Early 2000 & $44.45 \times 48.90 \times 55.9 \mathrm{~cm}$ & $\begin{array}{l}\text { Implemented four full } \\
\text { duplex channels and } \\
\text { could be remotely } \\
\text { controlled using the } \\
\text { Simple Network } \\
\text { Management Protocol via } \\
\text { an Ethernet interface. }\end{array}$ \\
\hline USRP & 2004 & $\begin{array}{l}\text { Fit in } 21 \times 17 \times 5.5 \mathrm{~cm} \\
\text { box }\end{array}$ & $\begin{array}{l}\text { Allows creating a } \\
\text { software radio using any } \\
\text { computer with a USB2 } \\
\text { port. Various plug-on } \\
\text { daughterboards allow } \\
\text { the USRP to be used on } \\
\text { different radio frequency } \\
\text { bands. }\end{array}$ \\
\hline
\end{tabular}

As shown in Table 1, SDR evolved from very large (and power hungry devices) to small man portable devices. Additionally, they evolved from very expensive prototypes to systems costing less than $1 \mathrm{k} €$. Today an SDR and computer are affordable as laboratory equipment 
for an undergraduate course; hence the time is ripe for the development of undergraduate laboratory course material.

\subsection{Modern Software Defined Radio}

\subsubsection{Hardware Architecture}

The basic hardware architecture of a SDR includes a radio front-end, modem, and application functions (see Figure 2; where the modem and application functions have been grouped together into a "Digital end" module). Additionally, there needs to be a means for connecting to network services and for remote management. The following subsections will discuss each of these elements of the SDR.

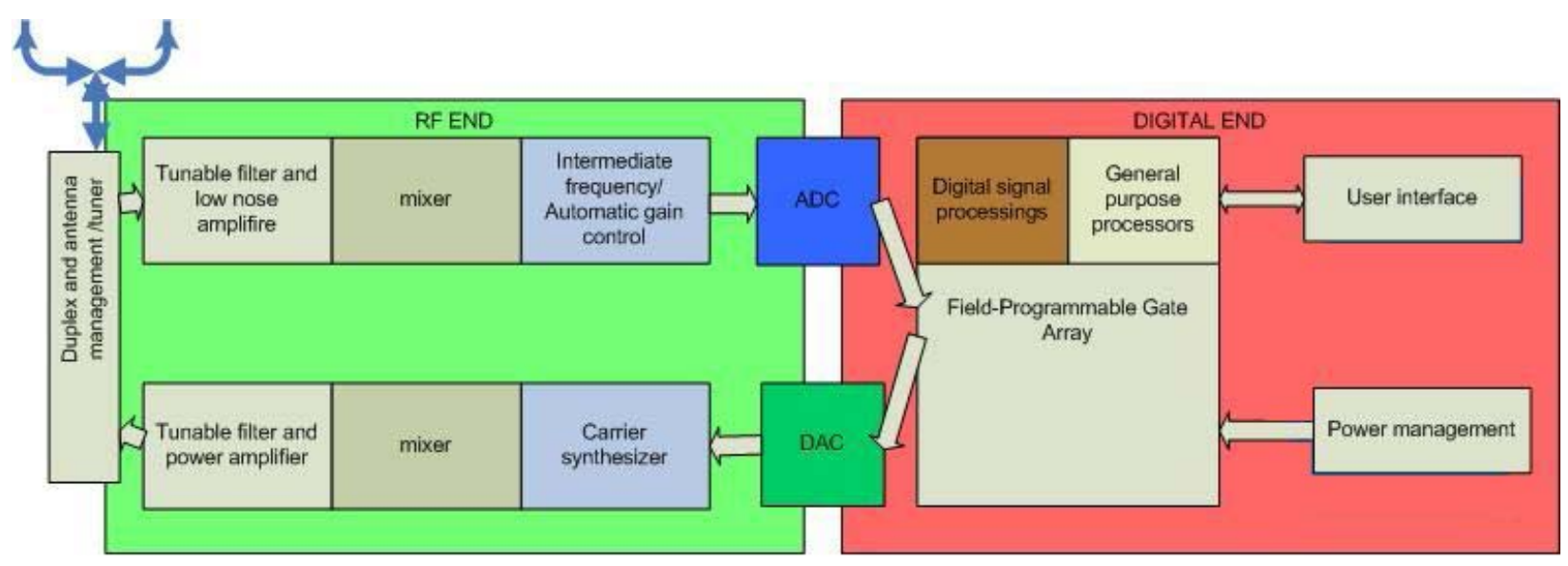

Figure 2: Basic hardware architecture of a modern SDR.

\subsubsection{RF Front-End}

The radio frequency (RF) front-end consists of functions to support transmit and receive modes. Note that some instance of a SDR might be receive mode only or transmit mode only. The receive mode utilizes:

- Antenna-matching unit

- Low-noise amplifier

- Filters

- Local oscillators

- analog-to-digital converters (ADCs).

This RF front end utilizes filters to reject (or reduce) undesired signals. An important part of this filtering is to prevent high frequency signals from being aliased into the digitized bandwidth of the ADC.

The transmit mode utilizes:

- Antenna-matching units

- Filters

- Local oscillator

- One or more digital-to-analog converters (DACs) 
The duplexer shown in Figure 2 is to enable the transmit and receiver subsystems to share an antenna, while avoiding overwhelming the receiver with the high power transmit signal.

\subsubsection{The modem}

The modulator/demodulator (modem) modulates signals to be transmitted or demodulates received signals. The modem process to receive signals is basically the inverse of the process used to modulate the signal to be transmitted. Figure 3 shows how this signal processing function is performed by the modem.

In Figure 3, bits are taken from a higher layer (such as a network layer packet) for transmission, grouped into frames, redundancy is added to enable error correction by the receiver, the bits are mapped to sample(s), and a specific wave is used to provide the selected representation of each symbol. Additional processing is performed to provide desirable physical properties and the signal may be multiplexed with other signals before being passed to the DAC.

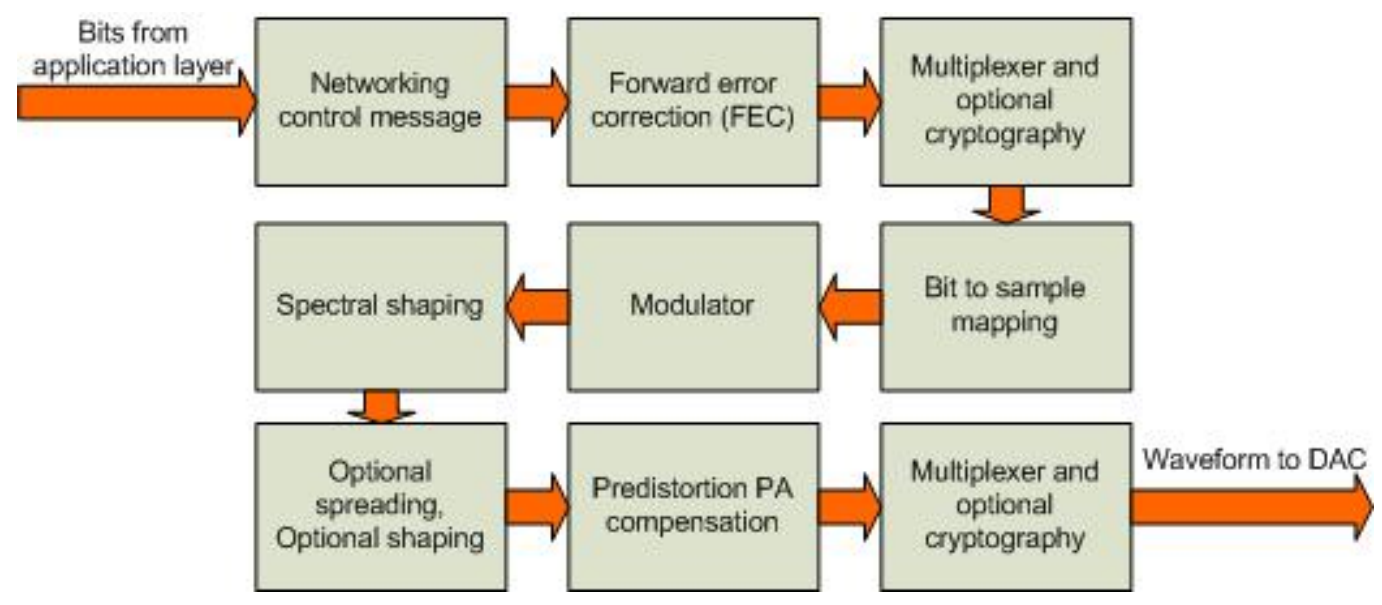

Figure 3: Signal processing block.

\subsection{Software Defined radio (SDR) and Software Radio (SR)}

There are many different definitions of the terms Software Defined radio (SDR) and Software Radio (SR). Walter Tuttlebee, et al. define SDR as "a radio in which the receive digitization is performed at some stage downstream from the antenna, typically after wideband filtering, low noise amplification, and down conversion to a lower frequency in subsequent stages - with a reverse process occurring for the transmit digitization. Digital signal processing in flexible and reconfigurable functional blocks defines the characteristics of the radio."[6]. These some authors define software radio (SR) by stating that as "technology progresses, an SDR can move to an almost total SR, where the digitization is at (or very near to) the antenna and all of the processing required for the radio is performed by software residing in high-speed digital signal processing elements.” [6].

In this thesis project we will mostly be concerned with SR as we perform most of the processing on the signal after it is available in a general purpose processor. This requires either a very high performance computer or limiting the bandwidth and signaling rates of the signals that we will deal with. 


\subsection{SDR Forum}

The SDR Forum was founded in 1996 by Wayne Bonser as "a non-profit international industry association dedicated to promoting the success of next generation radio technologies."[12]. SDR Forum members came from a number of different areas, including end customers, suppliers/manufacturers, standards organizations, academic institutions, and industry associations. The SDR Forum established an Educational Working Group to develop and deliver materials on a wide range of topics to facilitate the implementation of software defined radios. 


\section{The Universal Software Radio Peripheral (USRP)}

The Ettus Research Universal Software Radio Peripheral (USRP) [15] provides a low cost platform to develop SRs. The USRP has a Cypress FX2 USB 2.0 interface, four high speeds digital to analog converters, four high speed analog to digital converters, and a large Altera Cyclone field programmable gate array (FPGA) that interconnects all of the aforementioned devices. The USRP is shown in Figure 4 and schematically in Figure 5.

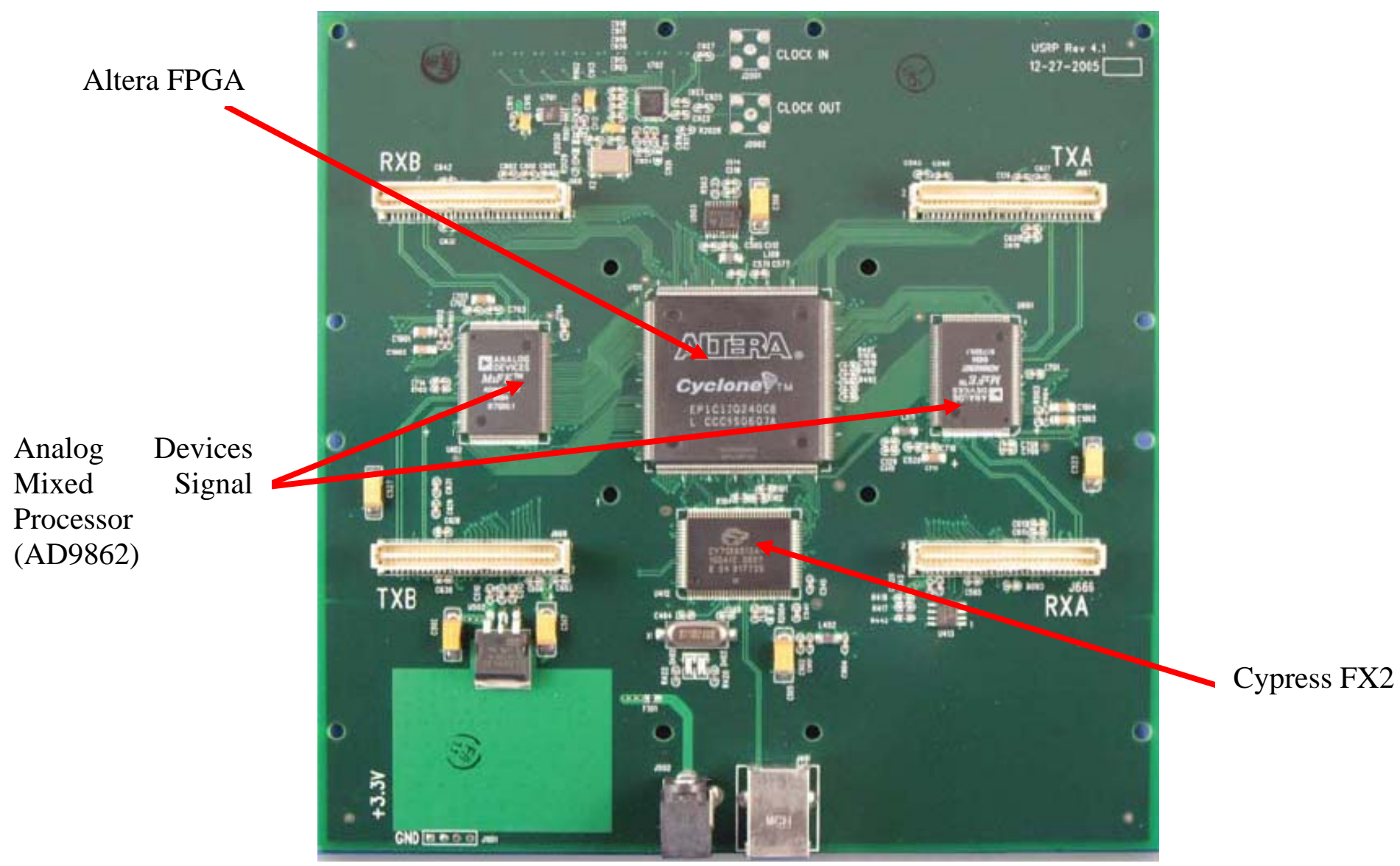

Figure 4: Universal Software Radio Peripheral (USRP)

Each AD9862 contains four ADCs. Programmable gain amplifiers, placed in-front of the ADCs provides input signal level adjustment. Further details of the AD9862 can be found at [17]. More specifically the USRP has two Analog Devices AD9862 chips for analog to digital and digital to analog conversions. These devices also support gain control for the analog path and signal processing for the digital path.

Each of ADCs runs at 64 Million samples per second (64 Msps) with 12 bits per sample, the DAC accept as input 14 bits per sample generating $128 \mathrm{Msps}$. As the maximum signaling rate of a USB 2.0 link is $480 \mathrm{Mbps}$, this means that we can not simply forward the entire received signal to an attached processor - nor can we receive a signal from an attached processor and output it directly via the DAC. Reducing the sample rate in the receive path and increasing the sample rate in the transmit path must be accomplished by the FPGA. Note that it is possible to run the ADC and DAC at lower rates. For some bandwidth signals the performance of the device may be sufficient to directly pass a digital version of the signal to the USRP and/or receive a digital version of the signal from the USRP. For example at 40 Msps it is possible to send 12 bit digitized data from the USRP to the host computer (if there 
is no traffic in the reverse direction). Similarly $48 \mathrm{Msps}$ at 10 bits per sample or 60Msps at 8 bits per sample might be possible.

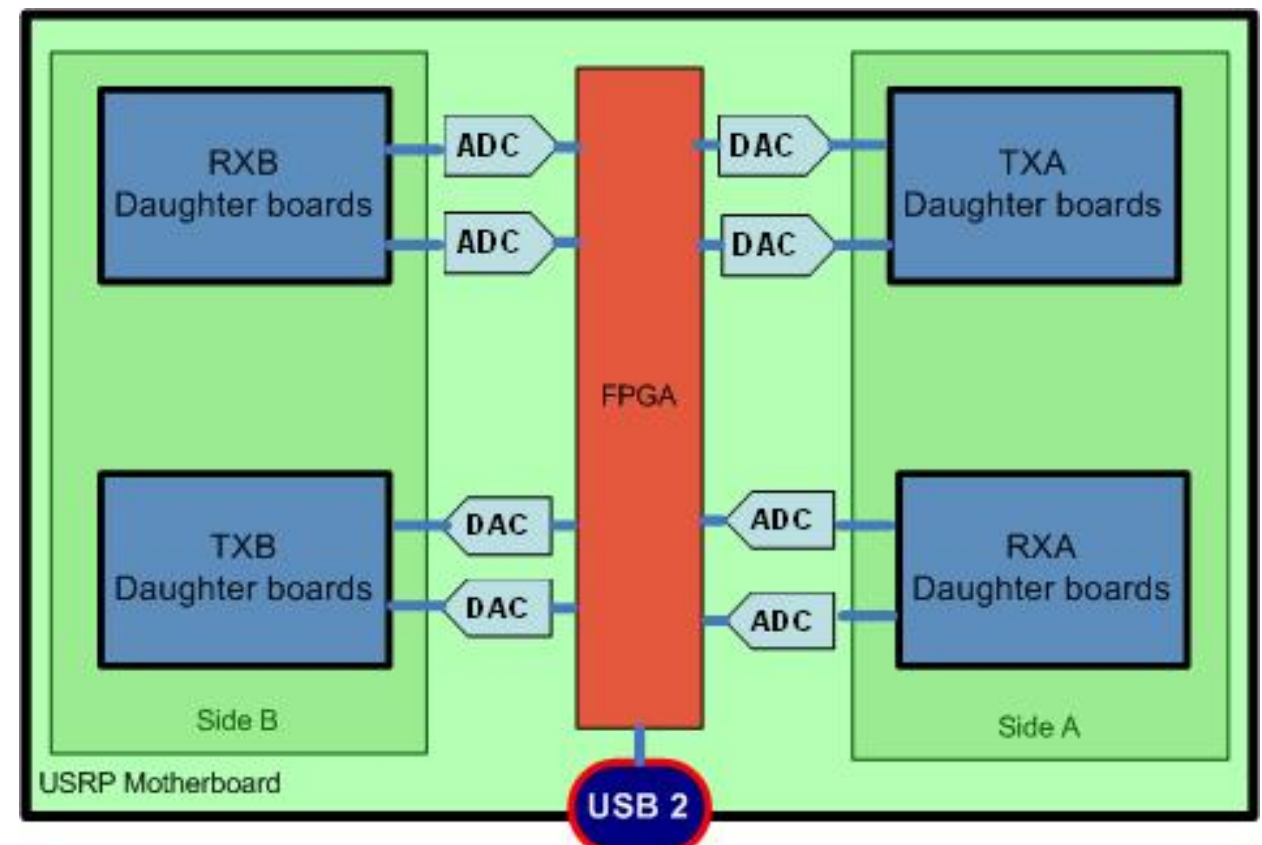

Figure 5: USRP Block Diagram

The Altera FPGA can be programmed using tools from Altera. The descriptions of the circuit to be mapped onto the FPGA are generally written in a hardware description language. In the case of the tools we have used, this language is Verilog (first standardized in IEEE 1364-1995 [18]; now IEEE P1800 [19]). The global clock frequency of the FPGA is 64MHz. This global clock frequency insures proper pipelining of everything within the FPGA.

\subsection{USRP Daughter boards}

There are four expansion slots on the USRP mother board. These enable a user to plug in up to two transmitter daughter boards and two receiver daughterboards. These daughters implement the specific radio frequency front end for a given range of frequencies. Thus the motherboard only performs baseband (or intermediate frequency) processing of the signals. On the USRP motherboard the transmitter expansion slots are labelled TXA and TXB, while the receiver expansion slots are labeled RXA and RXB. Each transmitter expansion slot has access to two high speed DACs; as the motherboard has four DACs with two connected to TXA and two to TXB. Each receiver expansion slot has access to two high speed ADCs, as the motherboard has four ADCs with two for RXA and two for RXB. This allows the system to simultaneously have two different RFs front-ends, enabling a given USRP to connect to two antennas for each of the two transmit and receive paths, for a total of four antennas in total. Note that there is no requirement that the receiver (or transmitter) daughter cards be for different frequencies, this flexibility might be used to have one daughter card tuned to one part of a frequency band while the other is turned to a different part of the same frequency band. Table 2 list a number of the different types of daughter boards that can be used with the USRP motherboard. Figure 6 shows a number of these daughter boards. 

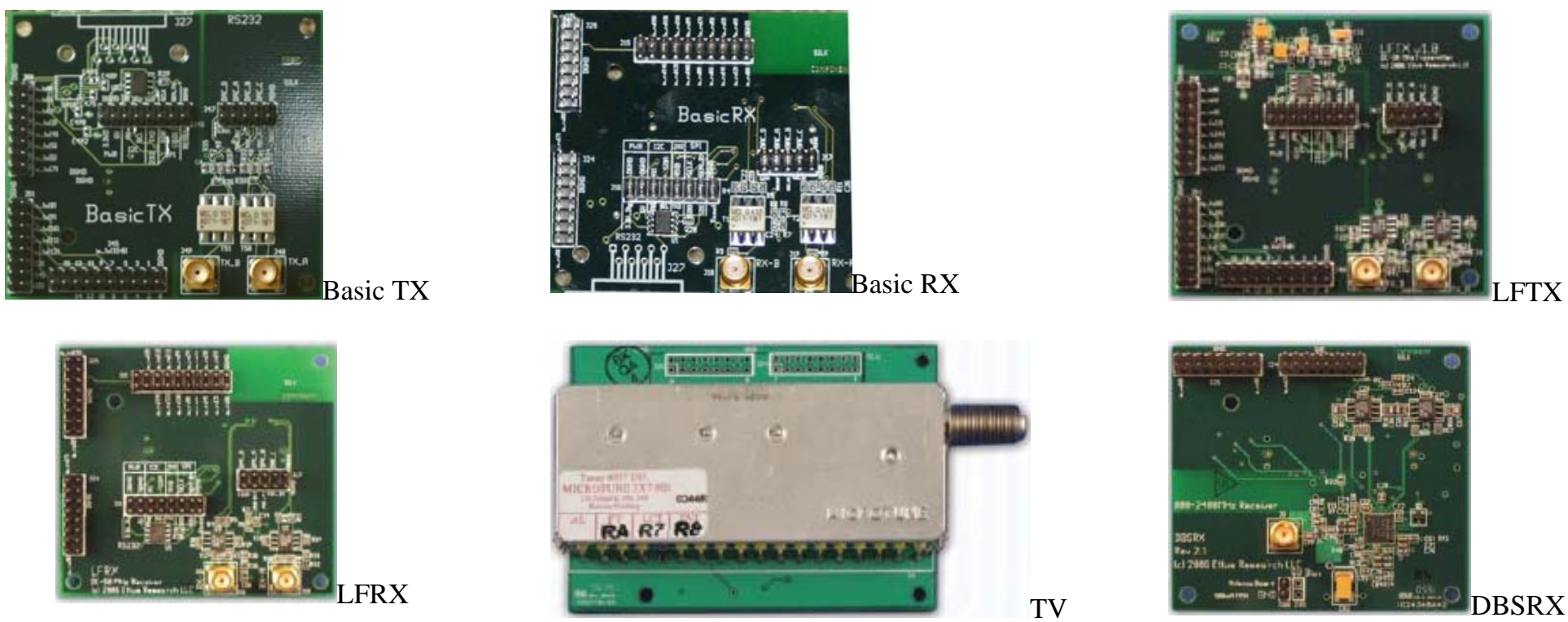

RX

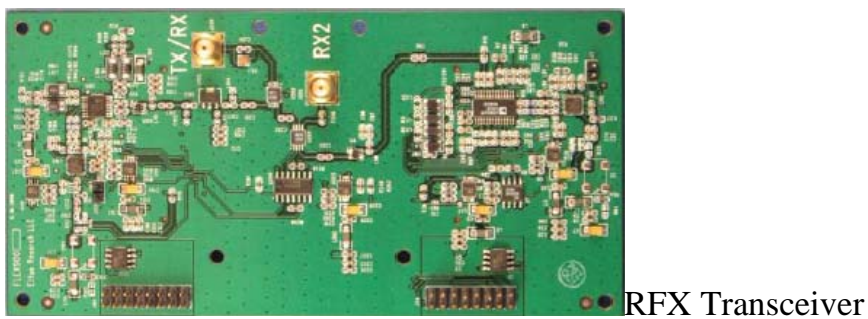

Figure 6: USRP Daughter boards

Table 2: USRP Daughter boards in use [15]

\begin{tabular}{|c|c|c|}
\hline Daughter board & Frequency Range & Note \\
\hline Basic TX & \multirow[t]{2}{*}{$1 \mathrm{MHz}-250 \mathrm{MHz}$} & Gives direct access to all signals on the daughter board interface. \\
\hline Basic RX & & $\begin{array}{l}\text { Designed for use with external RF and intermediate frequency } \\
\text { sources. }\end{array}$ \\
\hline LFTX & \multirow[t]{2}{*}{$\mathrm{DC}-30 \mathrm{MHz}$} & \multirow{2}{*}{$\begin{array}{l}\text { Frequency response extends down to DC. With a } 30 \mathrm{MHz} \text { low pass } \\
\text { filter to support antaliasing. }\end{array}$} \\
\hline LFRX & & \\
\hline TVRX & $\begin{array}{l}50 \mathrm{MHz}-860 \\
\mathrm{MHz}\end{array}$ & $\begin{array}{l}\text { Complete VHF and UHF receiver system based on a TV tuner } \\
\text { module. This is only a receiver and there is no corresponding } \\
\text { transmitter daughter card. }\end{array}$ \\
\hline DBSRX & $\begin{array}{l}800 \mathrm{MHz}-2.4 \\
\mathrm{GHz}\end{array}$ & $\begin{array}{l}\text { 3-5 dB noise. Covers many bands of interest for use for student labs } \\
\text { - since IEEE 802.11 WLAN and Bluetooth both use the } 2.4 \mathrm{GHz} \\
\text { band. Additionally, IEEE } 802.15 .4 \text { can use } 868.0-868.6 \mathrm{MHz} \\
\text { (Europe), 902-928 (North America), and 2.4-2.483.5 (worldwide). }\end{array}$ \\
\hline \multirow{5}{*}{$\begin{array}{l}\text { RFX; Series of } \\
\text { Transceivers }\end{array}$} & 400-500 MHz & RFX400 Transceiver, $100+m W$ output \\
\hline & $150-1450 \mathrm{MHZ}$ & RFX900 Transceiver, 200+mW output \\
\hline & $800-1000 \mathrm{MHz}$ & RFX1200 Transceiver, 200+mW output \\
\hline & $1.5-2.1 \mathrm{GHz}$ & RFX1800 Transceiver, $100+m W$ output \\
\hline & $2.3-2.9 \mathrm{GHz}$ & RFX2400 Transceiver, 20+mW output \\
\hline
\end{tabular}




\section{GNU Radio}

GNU Radio is free Python-based software architecture implemented to run on a Linux platform. More specifically, GNU Radio provides a collection of signal possessing blocks that support the USRP. This collection of signal processing blocks was developed by Eric Blossom in early 2000 [8]. Bruce A. Fette et al. argue that "GNU Radio in general is a good starting point for entry-level SDR and should prove successful in the market, especially in the amateur radio and hobbyist market.” [3]. Figure 7 illustrates how the GNU Radio signal processing blocks can be used together with the USRP.

The GNU Radio graphical user interface is written in Python. While a programmer could use any programming language to build an interface, the GNU Radio project recommends using wxPython [21] to maximize cross-platform portability.

The GNU Radio code is written in both $\mathrm{C}++$ and Python. The computationally intensive processing blocks are implemented in $\mathrm{C}++$, while Python is used for developing applications that sit on top (and control) these blocks. The GNU radio code assumes that the FPGA has already been programmed with a configuration suitable for use by the GNU radio code.

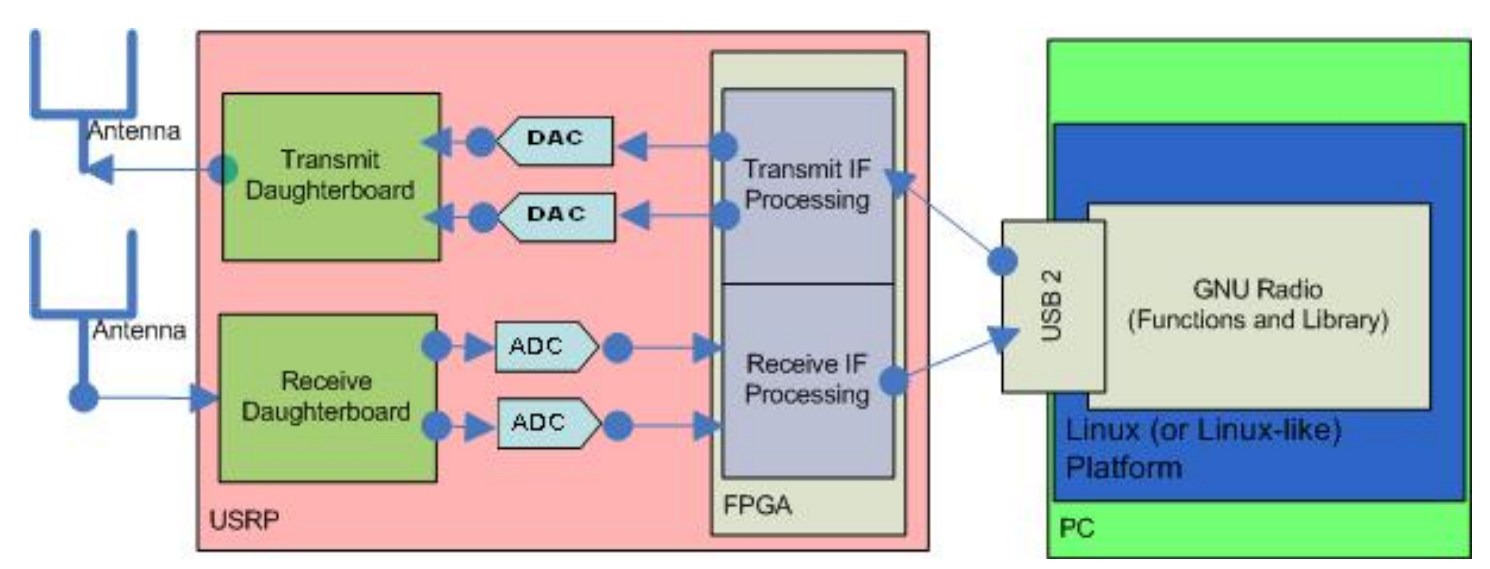

Figure 7: A basic SDR system based on GNU Radio and USRP

\subsection{Installing the GNU Radio}

This section describes how to build GNU Radio version 3.2.2 - released on July 15, 2009. In this thesis we experienced problems installing GNU Radio as described in this release's build guide [25]. The problems are:

1- SVN version (svn co http://gnuradio.org/svn/gnuradio/branches/releases/3.2 gnuradio) gives errors on installation. Instead, we used the tarball file to get the final stable release (ftp://ftp.gnu.org/gnu/gnuradio/gnuradio-3.2.2.tar.gz).

2- GNU Radio version 3.2.2 needs boost library version 1.35 or later which is not part of Fedora 10 or Ubuntu 8.04. The build guide describes how to install boost version 1.37. The build guide gives an example of installing boost in /opt/boost_1_37_0 by doing the following: 
\$ BOOST_PREFIX=/opt/boost_1_37_0

\$./configure --prefix=\$BOOST_PREFIX --with-libraries=thread,date_time,program_options

After this you should install GNU Radio:

\$ export LD_LIBRARY_PATH=\$BOOST_PREFIX/lib

\$./configure --with-boost=\$BOOST_PREFIX

Unfortunately, following these instructions will give an error that GNU Radio can not find boost version 1.35 or later. This can be fixed by installing boost in the default directory /usr/local/ ; thus, our installation solution is to install boost by saying:

\$ ./configure --prefix=/usr/local/ --with-libraries=thread,date_time,program_option

Now GNU Radio can be installed by simply saying:

\$./configure

\subsubsection{Installing GNU Radio on Fedora 10}

Preparing Fedora 10 for installations. Install basic requirements for building GNU Radio by running the following:

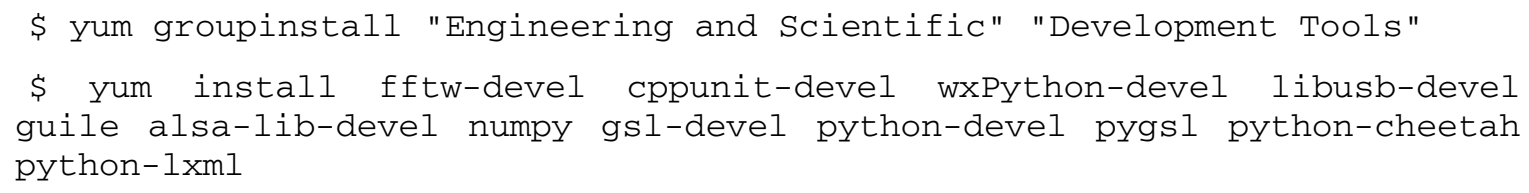

Build the firmware for the microcontroller on the USRP by running:

\$ yum install sdcc

Add /usr/libexec/sdcc to your PATH before building GNU Radio by running:

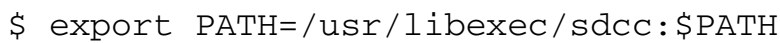

Install the HTML documentation generator by running:

\$ yum install xmlto graphviz

Install the Qt plotting tools by running:

\$ yum install qt4-devel qwt-devel qwtplot3d-qt4-devel

Set the PYTHONPATH environment variable to the appropriate value. This can be done by the following two steps. First which determine Python version you are using. This can be done by running:

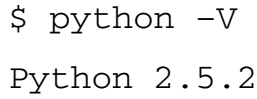

Second set the PYTHONPATH environment variable to the appropriate value for this version. This can be done by the following ( be careful of the Python version):

\$ export PYTHONPATH=/usr/local/lib/python2.5/site-packages

Download and install boost into /usr/local/

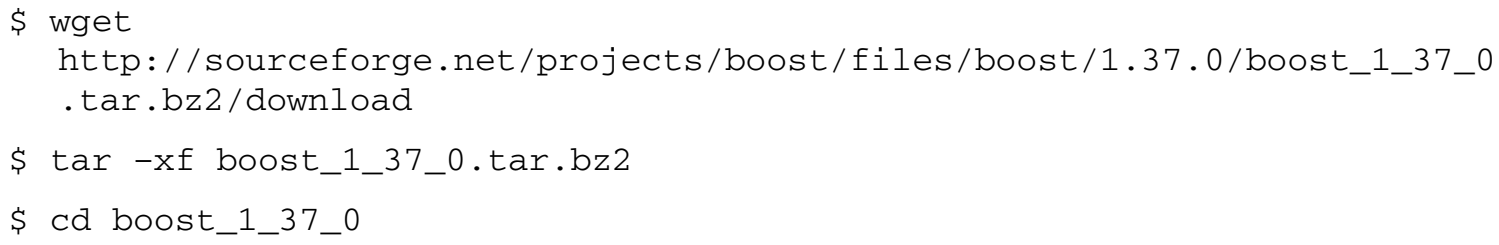




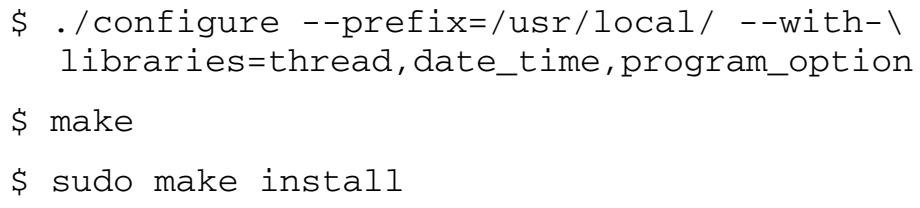

Download and install GNU Radio

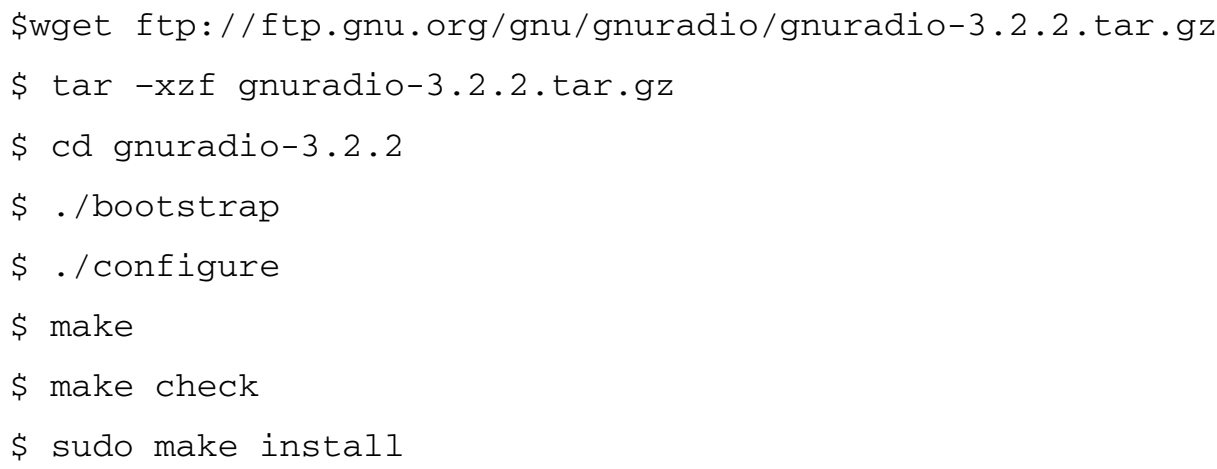

\subsubsection{Installing GNU Radio on Ubuntu 8.04}

To prepare Ubuntu 8.0410 for installation of the GNU Radio software you need to install a nuber of modules. Add the following repositories in the source packages (see Figure 8)”
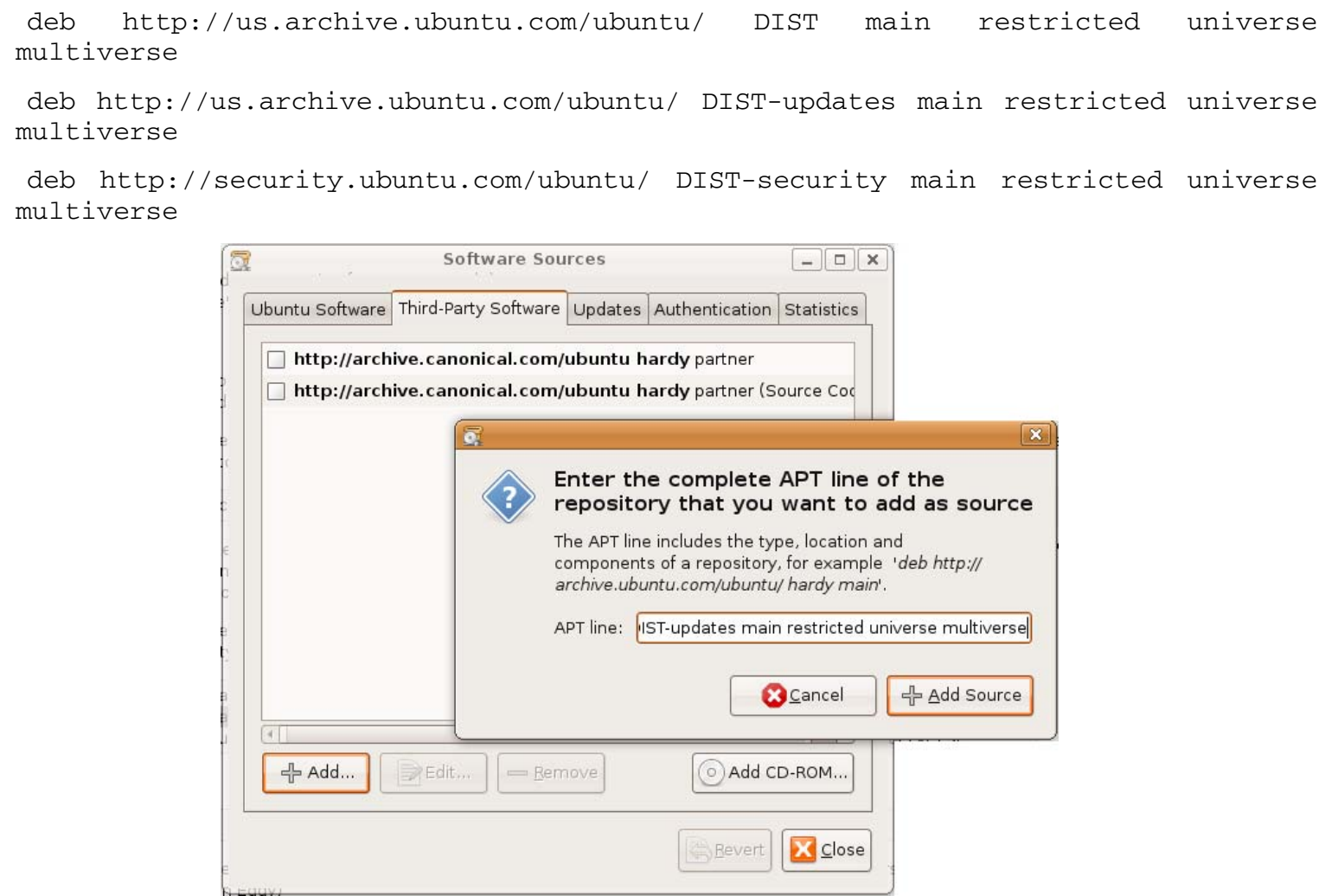

Figure 8: Adding Repositories Using Software Sources in Ubuntu 8.04

Update the package management system

\$ sudo apt-get update 
Install the required packages

\$ sudo apt-get $-y$ install swig g++ automake1.9 libtool python-dev fftw3-dev /

libcppunit-dev sdcc libusb-dev libasound2-dev libsdl1.2-dev /

python-wxgtk2.8 subversion guile-1.8-dev libqt4-dev python-numpy-ext /

ccache python-opengl libgsl0-dev python-cheetah python-1xml doxygen /

ccache python-opengl libgslo-dev python-cheetah python-lxml doxygentools

Install optional packages

sudo apt-get $-y$ install gkrellm wx-common libwxgtk2.8-dev alsa-base autoconf xorg-dev g77 gawk bison openssh-server emacs cvs usbview octave

Download and install boost into the /usr/local/

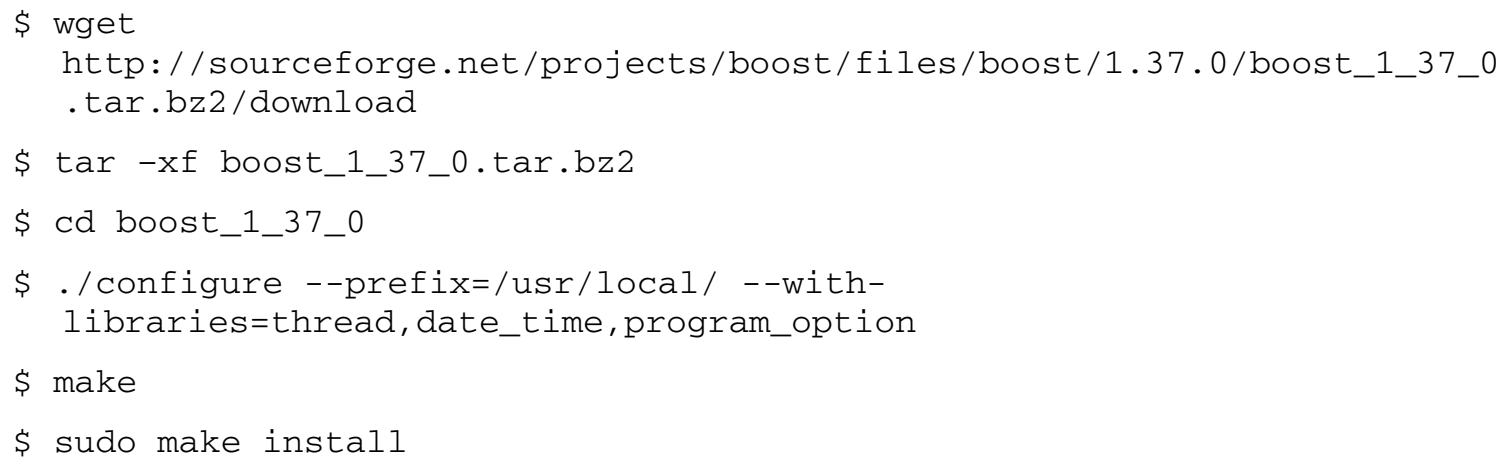

Download and install GNU Radio

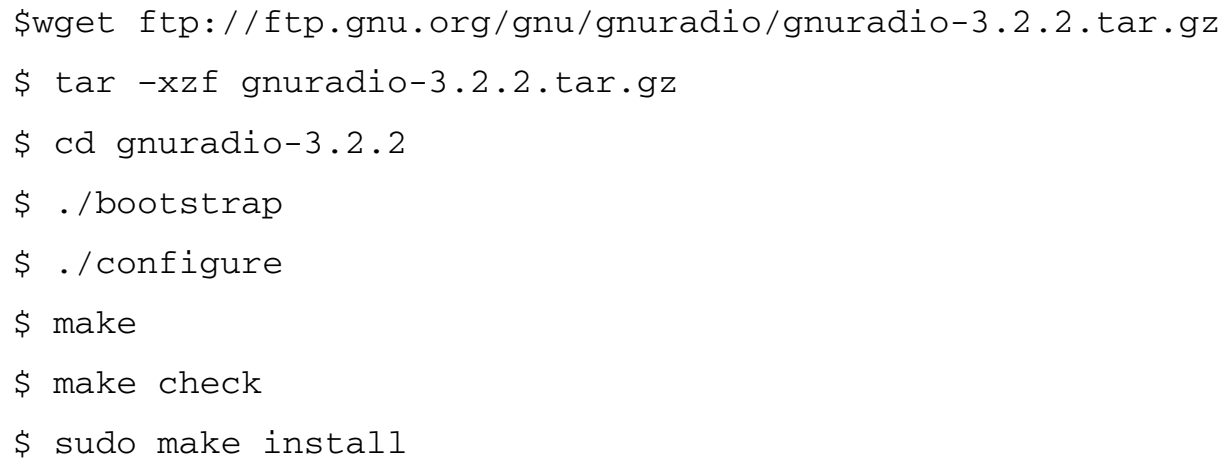

Provide non-root user access to the USRP

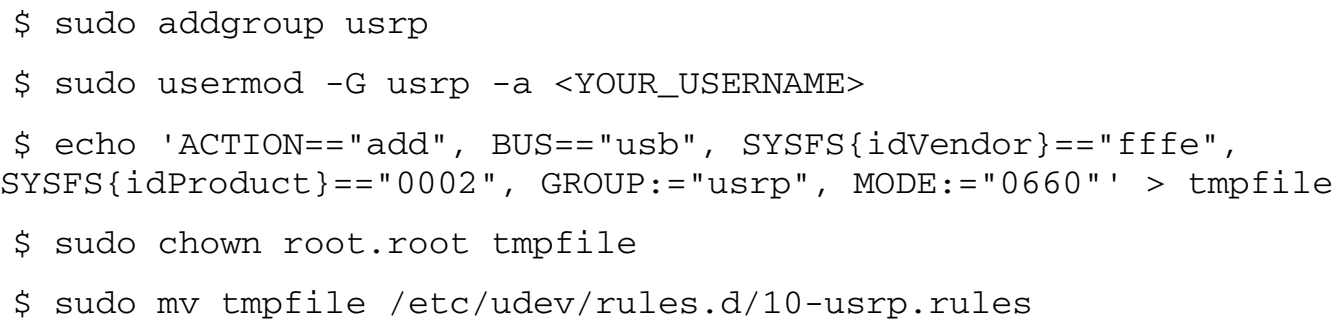

\subsection{GNU Radio Python Applications}

The basic concepts underlying the GNU Radio are flow graphs and blocks (nodes of the graph). The blocks carry out the actual signal processing (see section 4.3). The data passed between these blocks could be of any kind. Figure 9 shows an example of a dial tone flow 
graph (dial_tone.py). This code is one of the GNU Radio examples. The source code is shown in Code example 1.

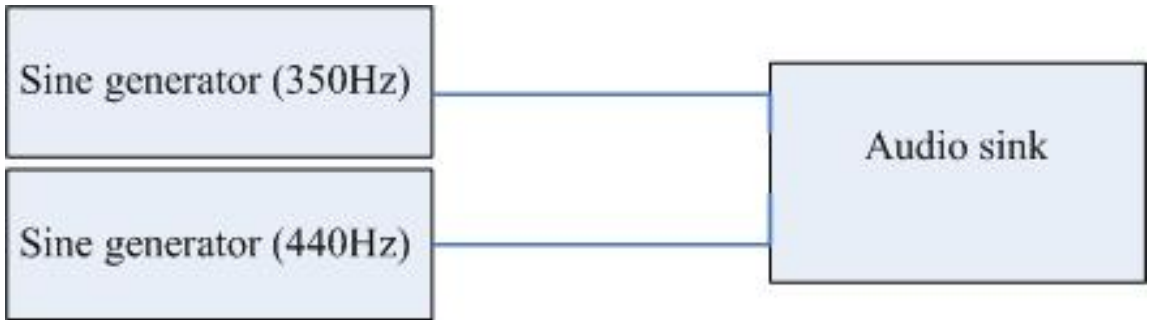

Figure 9: Flow Graph to generate a Dial Tone

In this example there are two sources. These sources generate $350 \mathrm{~Hz}$ and $440 \mathrm{~Hz}$ sine waves (in order to make an American dial tone). These sources are connected to a single audio sink with two inputs (the signal passed to one input is output by the audio sink on the left channel of the sound card, while the input to the second input is output on the right channel of the sound card). The result will be you will hear the two tone dial tone.

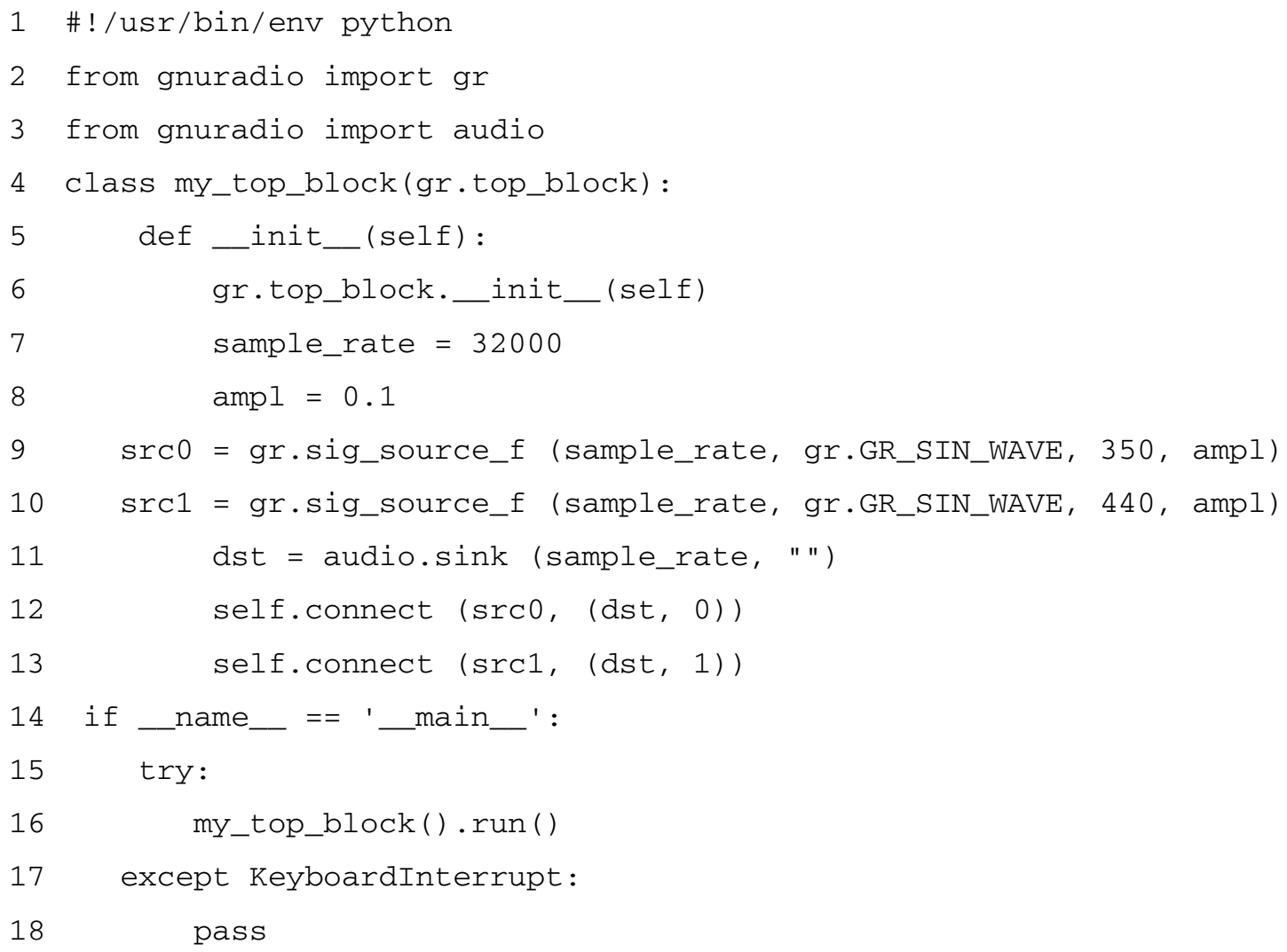

\section{Code example 1: dial_tone.py}

Line 1 tells the shell that this is Python file and that it should use the Python interpreter to execute it. On lines 2 and 3, the import command imports the GNU Radio (gr) and audio modules from the GNU Radio. The gr module must be imported to run a GNU Radio application. The audio module loads an audio device block (to input or output audio from a sound card and to control this audio device). Lines 4 begins the definition of my_top_block class which is derived from gr.top_block (a subclass of $\mathrm{gr}$ ), this is a flow graph container. The class my_top_block is defined from line 4 to 13. Line 5 defines a function (the constructor of the class) my_top_block ( _ init__ ). The function is realized in line 6 by calling the parent constructor, then in line 7 setting the sample_rate variable which controls the sampling rate of the signal is set, and line 8 sets the ampl variable which controls the 
amplitude of the signal. The dial_tone example (see Figure 9) contains three blocks and two edges (connections), line 9 defines a signal source $(s r c 0)$ which generates a sine wave at 350 $\mathrm{Hz}$, 32k sampling rate, and 0.1 amplitute. While line 10 defines a signal source ( $\operatorname{src} 1)$ which generates a sine wave at $440 \mathrm{~Hz}, 32 \mathrm{k}$ sampling rate, and 0.1 amplitude. The ' $f$ sufix of gr.sig_source $f$ indicate that the source signal is a floating point value. Line 11 defines the destination (dst) asan audio sink -this can be used to send/receive audio signals to a sound card and to control this sound card. Lines 12 and 13 connect the block. The connect syntax depends on the number of outputs of block1 and block2, the syntax is self.connect(block1,block2,block3....), this would indicate that block1's output should be connected with block2's input, and block2's output should be connected to block3's input. The statements try and except on lines 15 and 17 stop the Python running program when the user press Control-C on the keyboard. Line 14 indicates that if this code is the only module being executed by the Python interpreter that the following code should be executed -- this will cause my_top_block to be executed.

\subsection{GNU Radio Signal Processing Blocks}

The GNU Radio project provides many signal processing blocks (implemented in $\mathrm{C}++$ ) as a library and supports the ability to be establish connections between these blocks. The programmer develops a radio by building a flow graph in which the signal processing blocks are represented as vertices and the data flow between them is represented as edges. Blocks' attributes specify the number of input ports and/or output ports and the data type (for example: short, float, and complex) for this port. Blocks may be built outside the GNU Radio core, then loaded as a shared library. Python dynamically loads shared library blocks using import specifications. Simplified Wrapper and Interface Generator (SWING) can be used to build the connections to allow code to be called from Python. GNU Radio includes a basic set of signal processing blocks that programmers can import into their applications. 
Table 3 shows these blocks. 
Table 3: GNU Radio Signal Processing Blocks

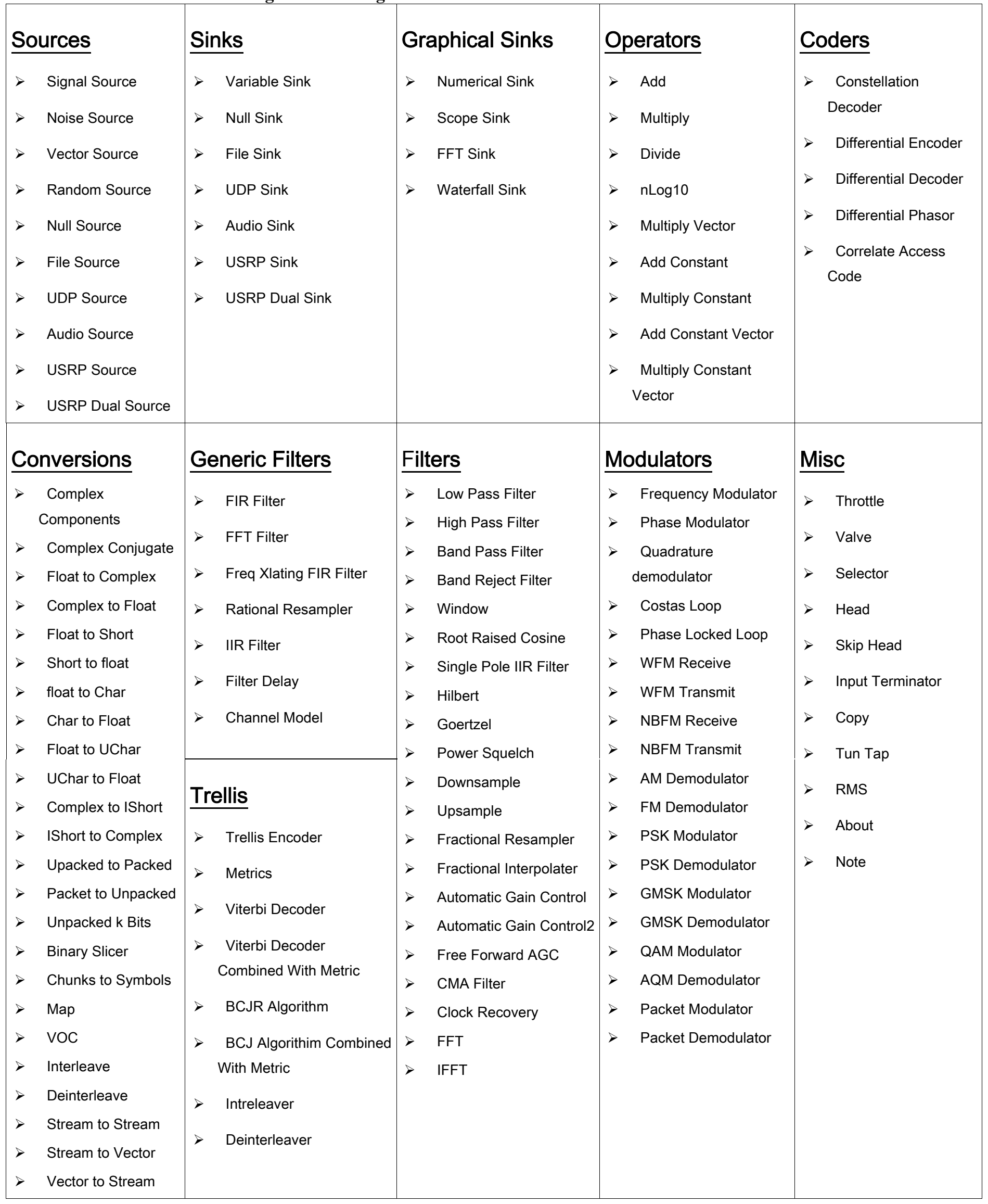


The gr_block $\mathrm{C}++$ class is the base of all classes. Writing a signal processing block involves writing the following files:

1-.$h$ file: Creates libraries of codes.

2- .cc file: Defines a new class and allows it to be called from python.

3- .i file: Tells SWIG how to build the connection.

The GNU Radio installation involves installing autotools (see 4.1), which includes autoconf, automake, and libtool tools. These tools facilitate portability across a variety of systems, and are used to generate Makefiles, read configure.ac, and producing a configure shell script. Makefile.am specifies the libraries to be used and is read by automake to generate a Makefile.in file. The directory layout of a new signal processing block is shown in Table 4.

Table 4: Directory layout of a new signal srocessing block [22]

\begin{tabular}{|l|l|}
\hline Directory/File name & Description \\
\hline Your_dir/Makefile.am & Top level Makefile.am \\
\hline Your_dir/Makefile.common & Common fragment included in sub-Makefiles \\
\hline Your_dir/bootstrap & Runs autoconf, automake, libtool first time through \\
\hline Your_dir/config & Directory of m4 macros used by configure.ac \\
\hline Your_dir/configure.ac & Input to autoconf \\
\hline Your_dir/src & Source directory \\
\hline Your_dir/src/lib & C++ code goes here \\
\hline Your_dir/src/lib/Makefile.am & \\
\hline Your_dir/src/python & Python code goes here \\
\hline Your_dirsrc/python/Makefile.am & \\
\hline Your_dir/src/python/run_tests & Script to run tests in the build tree \\
\hline
\end{tabular}

\subsubsection{Creating a Simple Signal Processing Block}

In this section we will describe how to write a simple signal processing block that calculates the square of a single input floating point value. Writing the block involves creating.$h$, .cc, and.$i$ files. In this example, the block will be named howto_square_ff, while the block in the Python module ends in the string gnuradio.howto. The gr_block.h (see Appendix A) includes a general_work method which is responsible for the actual signal processing, the simple signal processing block overrides the general_work code. The following code and description show the howto_square_ff.h, howto_square_ff.cc, and howto.i file. 


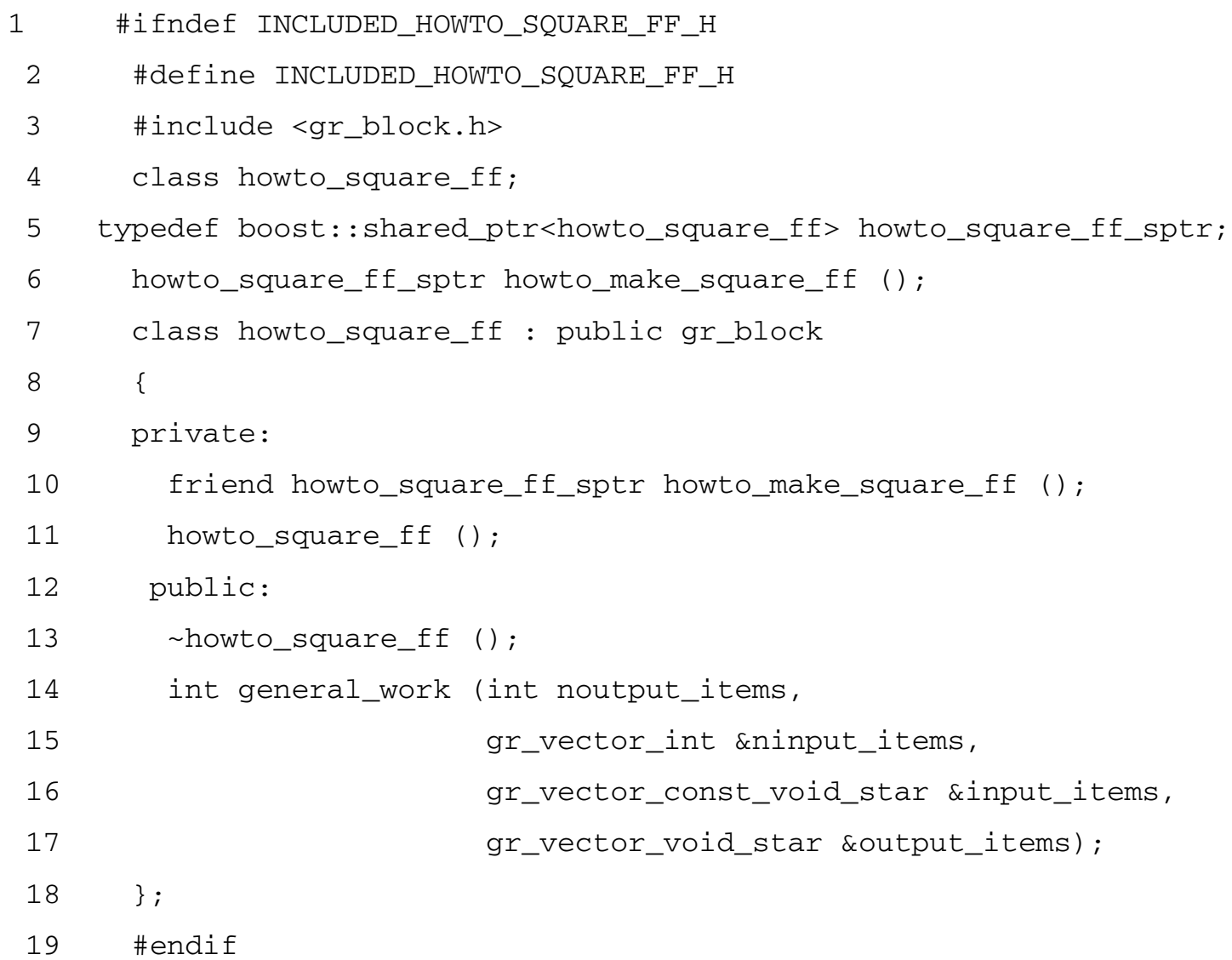

\section{Code example 2: howto_square_ff.h}

Lines 1 and 2 in the code prevents multiple reference if this should be included more than once, line 3 includes the gr_block.h library file, the class howto_square_ff is defined in line 4. Line 5 defines that to access the gr_block.h we will use boost::shared_ptr which is helpful in a $\mathrm{C}++/$ Python environment to dynamically allocate objects and automatically delete pointers at the appropriate time [23]. Line 6 defines howto_make_square_ff as a public interface. The friend declaration on line 10 allows howto_make_square_ff to access the private constructor. Howto_square_ff is defined as a private constructor on line 11, while $\sim$ Howto_square_ff on line 13 is public destructor. Lines 14 to 17 override the general_work method which is defined in gr_block.h. Finally, line 19 ends the INCLUDED_HOWTO_SQUARE_FF_H conditional block. 


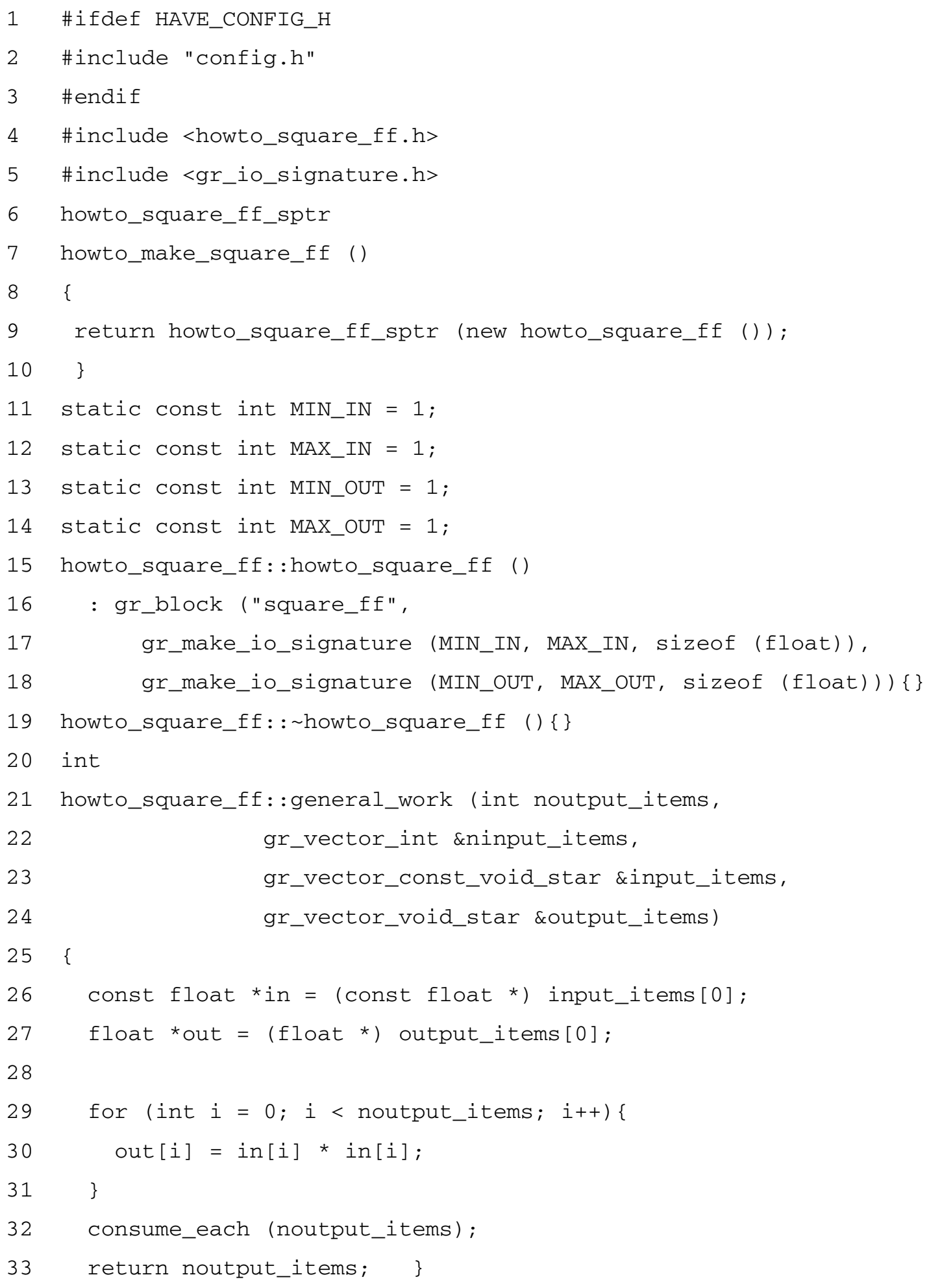

Code example 3: howto_square_ff.cc

The file config.h (line 2) contains probing results, and were generated by configure. Lines 6 to 10 create a new instance of howto_squire_ff, return a boost shared_ptr. Lines 11 to 14 specify constraints on the maximum and minimum input and output streams (and the width of the data values for these streams), in this simple signal processing block only one 
input and one output are accepted and the values are sizeof(float) bytes wide. Lines 15 to 18 define the private constructor, and lines 19 to 31 show the virtual destructor that calculates the square of a single input floating point number. Lines 32 and 33 tell the run time system how many input items will be consumed on each input stream and how many output items will be produced.

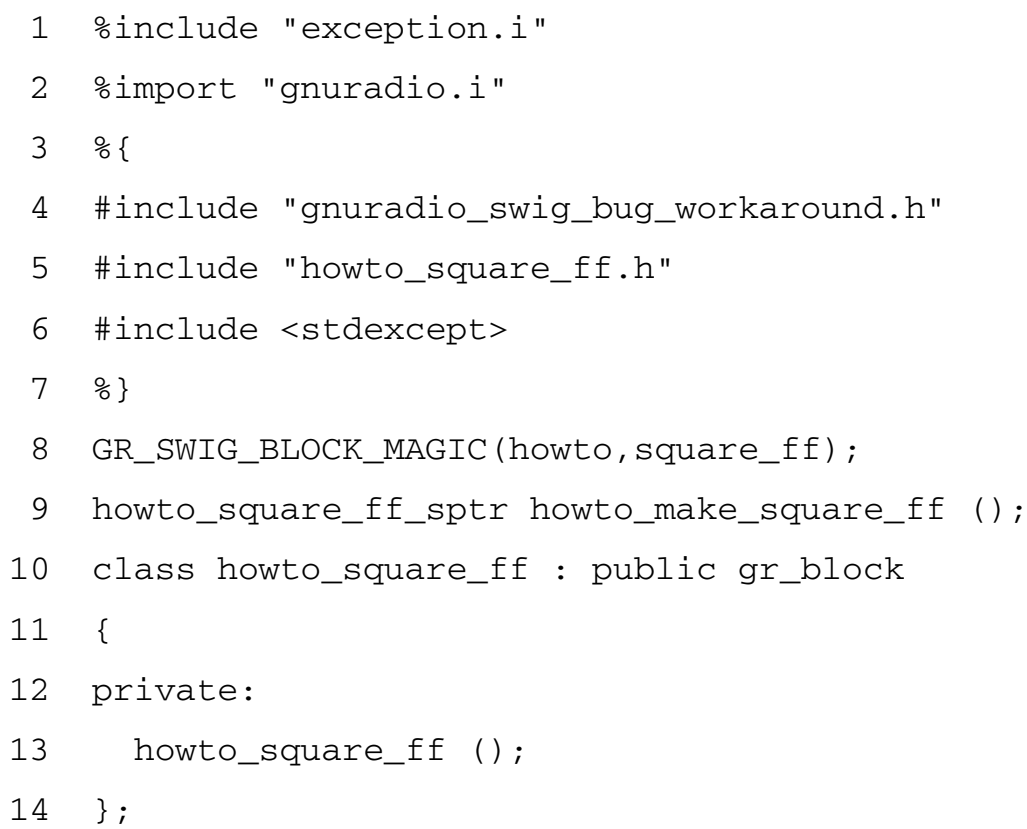

Code example 4: howto. $i$

Line 6 defines a mandatory bug fix. The arguments on line 8, howto: is the package prefix, and square_ff: is the name of the class without the postfix prefix.

The file Makefile.am is needed to complete the simple signal processing block. This file is located in the "Your_dir/src/lib/" directory (see Table 4). This file will be used to build a shared library from the source file and includes additional rules to use SWING.

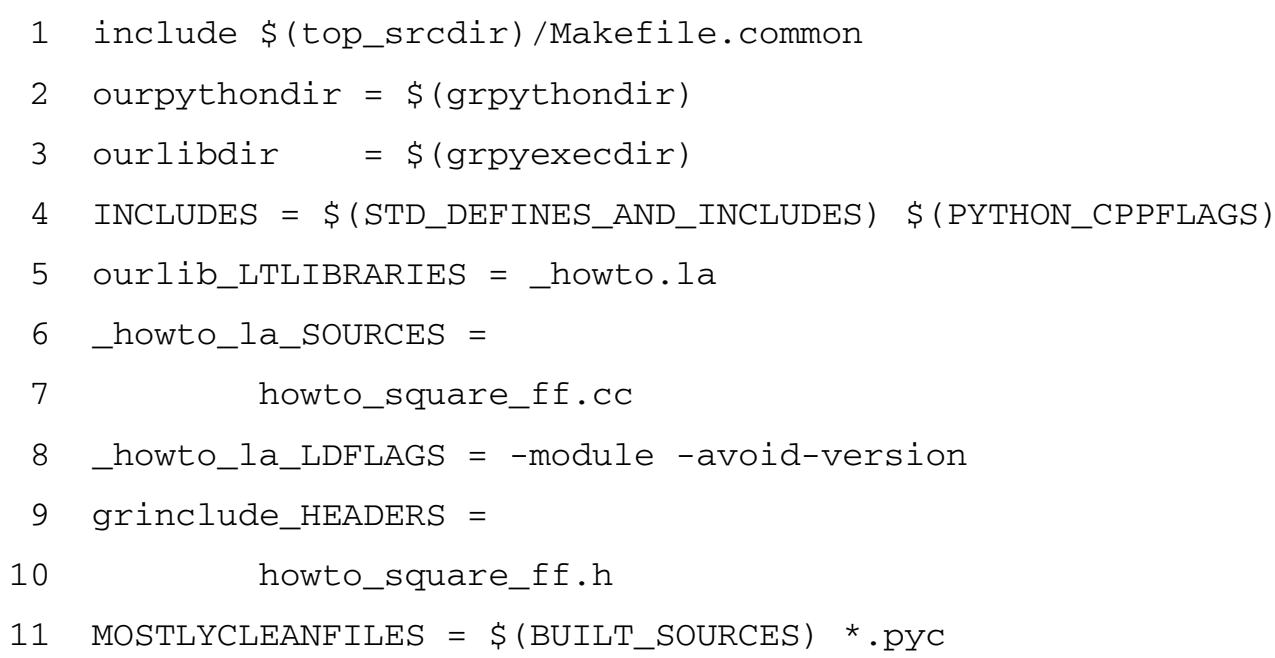

Code example 5: src/lib/Makefile.am 


\section{Laboratory Experiments}

This chapter describes some of the laboratory exercises that have been designed during this project. The first exercise concerns simplex data transmission, the second exercise concerns simplex voice transmission, the third exercise introduces carrier sense multiple access, the fourth exercise realize a Bluetooth sniffer (IEEE 802.15.4 sniffer), the fifth exercise realize a full IEEE 802.11 implementation.

\subsection{Experiment 1: Simplex data transmission}

In this exercise we will learn how simplex data communication can be implemented. In this case there will not be any feedback from the receiver packets arrival of the packets at the receiver. The transmitter sends 5 packets, then waits one second and sends the next 5 packets. The equipment required for this exercise is a PC, together with one USRP, Basic TX, Basic $\mathrm{RX}$, and an RF cable. Based on the exercise plan, the following objectives were developed for the simplex data transmission.

- Objective 1: Learning how to assemble and disassemble a simple header

- Objective 2: Learning how to generate and send a signal packet.

\subsubsection{Requirements}

- One USRP; with one Basic RX and one basic TX installed.

- $\quad$ One PC; GNU Radio installed.

- One RF Cable

\subsubsection{Simplex data transmission implementation}

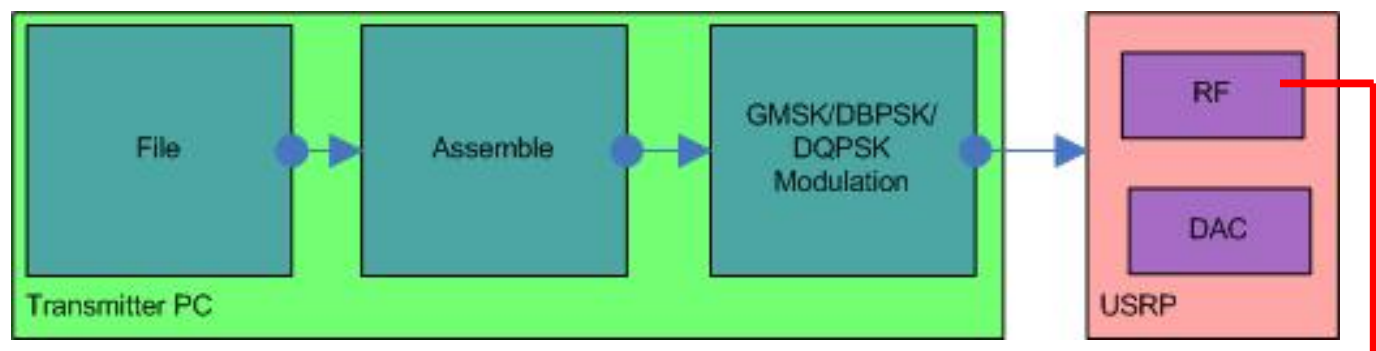

RF cable

Figure 10: Simplex data transmitter

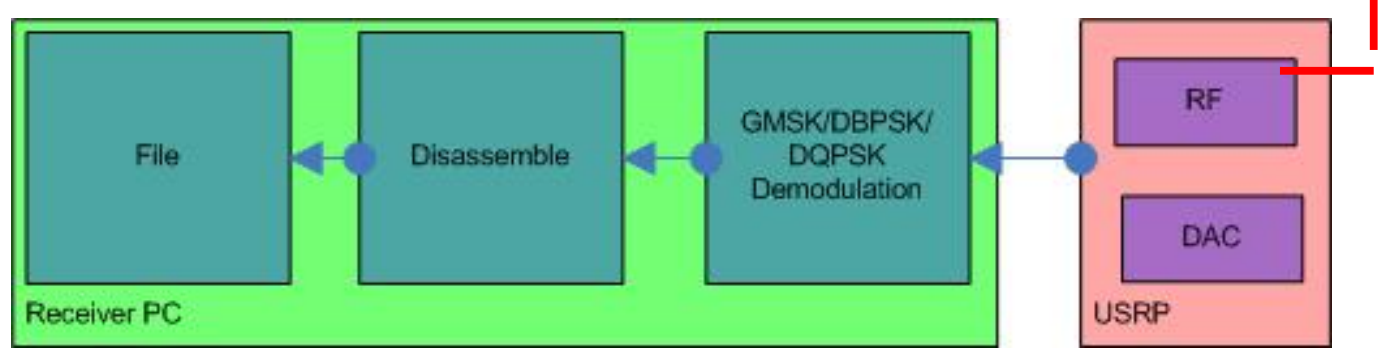

Figure 11: Simplex data receiver

Note that in the figure the first (green) box shows the code that is running on the PC, while the next (pink) box shows the USRP, and RF cable connected the transmitter with the 
receiver (rather than using two antennas). The reasons that we do not use an antenna for this experiment are we do not want to radiate energy into the world nor receive signals (other than from the transmitter). Using a cable also allows multiple students to carry out this laboratory exercise at the same time without interfering with each other.

\subsubsection{Understanding the code}

The code of this exercise is part of the GNU Radio examples located in (see Appendix B.1 benchmark_tx.py and Appendix B.2 benchmark_rx.py ; benchmark_tx.py and benchmark_rx.py and located in:

gnuradio-3.2.2/gnuradio-examples/python/digital/benchmark_tx.py

gnuradio-3.2.2/gnuradio-examples/python/digital/benchmark_rx.py

the file benchmark_tx.py is the transmitter code, while the file benchmark_rx.py is the receiver code.

\subsubsection{Transmitter}

The file benchmark_tx.py generates packets and frames in the format as shown in Figure 12. The size of the frame specified by the user. The software running in the PC generates the packets and frames and passed them via the USB interface to USRP.

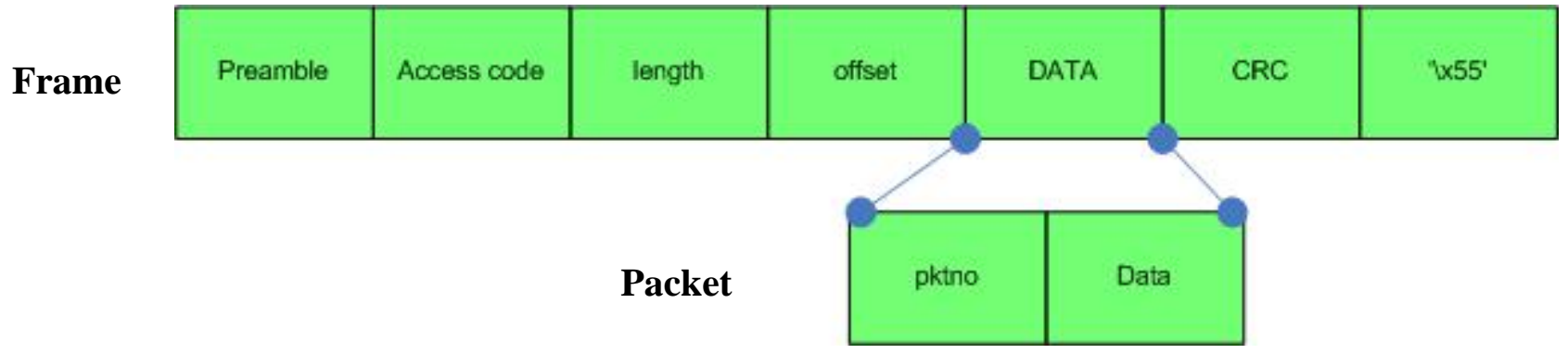

Figure 12: Simplex data transmission; showing the relation between the packet and the link frame

The following code describes how to generate and send packets. There are two options for the source of the file; the file is either defined by the user or generated by the program. The transmitter waits one second after sending five packets; then repeats this process. The default packet size (pkt_size) is 1500 bytes which includes two bytes containing the packet number ( show as pktno in the figure above). These two extra bytes, means that each frame contains pkt_size -2 bytes of data. Each frame ends with a octet containing $0 \times 55$ - this is used as a marker to terminate the frame. The preamble is used by the receiver to recognize the start of new frame. In Code example 6 we can see struct function which is responsible for generating packets in the format represented in Figure 12. struct is Python function, the "!" indicates that the byte order of the packed data is network (big-endian). The struct.pack() is used to packing a packet, while struct.unpack() is used to unpack packet (see Code example 6 ). The " $\mathrm{H}$ " format character in the struct function means the conversion between $\mathrm{C}$ language and Python values should be obvious given its type (unsigned short $C$ type to integer Python type). 


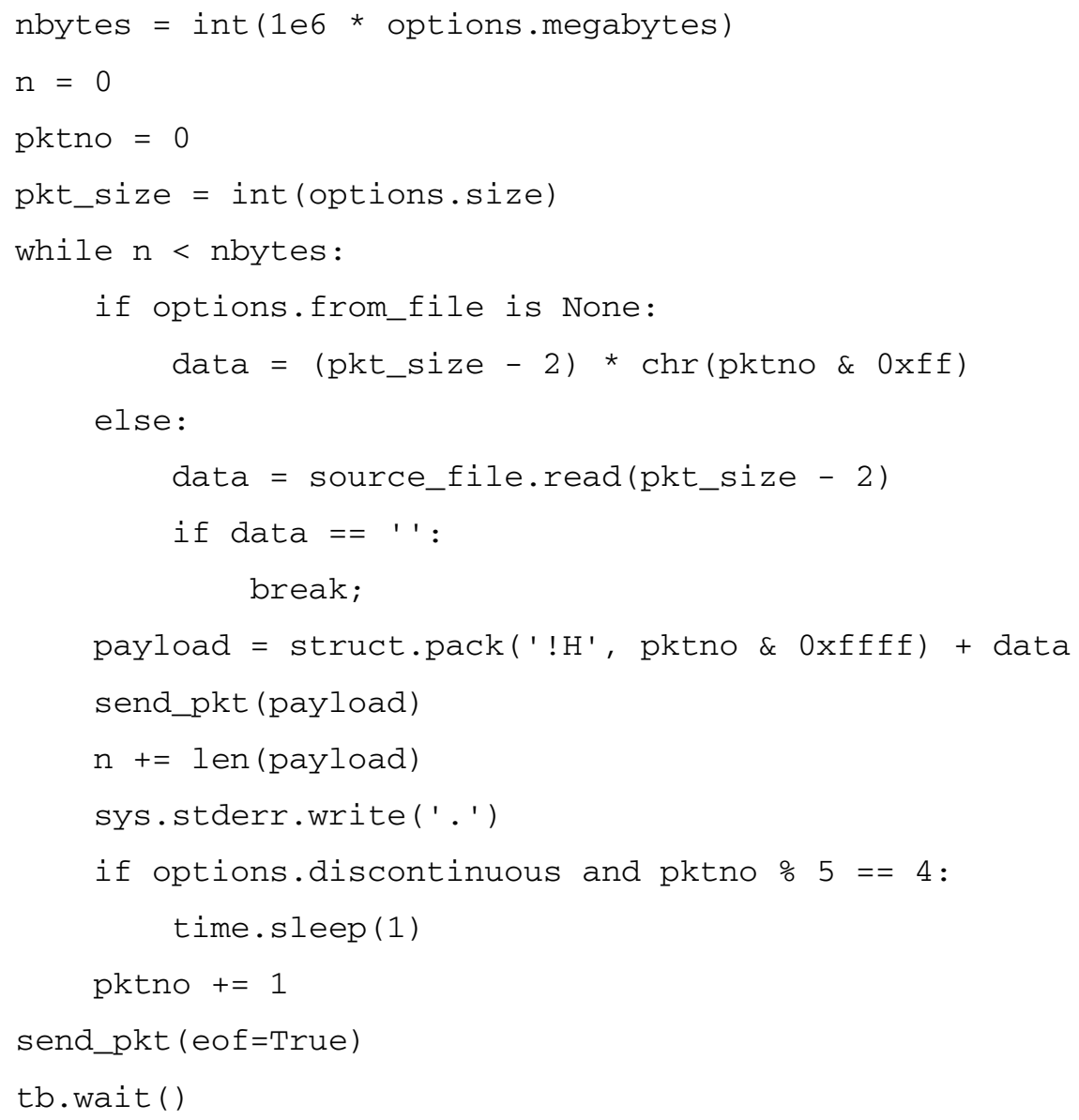

Code example 6: benchmark_tx.py; generate and send a packet, sleep after sending 5 packets

Table 5 shows the transmitter options when running the code.

\section{Table 5: The transmitter options}

\section{Options Descriptions}

-m The modulation choice. The user can choose between GMSK, DBPSK, and DQPSK modulations. The default is GMSK. Details of these different modulations can be found on [34].

-s The packet size choice. The user can define packet size he desire, the default packet size is 1500 bytes.

-M Sets the number of megabytes to send. This option tells the program to generate a file of indicated size.

-f Defines the desired frequency. This frequency must be set to the same value in both the transmitter and receiver.

\subsubsection{Receiver}

The program implemented by benchmark_rx.py listens for incoming packets and prints a summary of each packets, and checks for errors in each packet. In the printed summary the strings "True" or "False" indicates that the CRC of the DATA is correct ("True") or wrong ("False"). The packet contains the field "pktno" and "payloaad". Code example 7 (from benchmark_rx.py ) encapsulates the packet from the frame and prints a packet summary. 


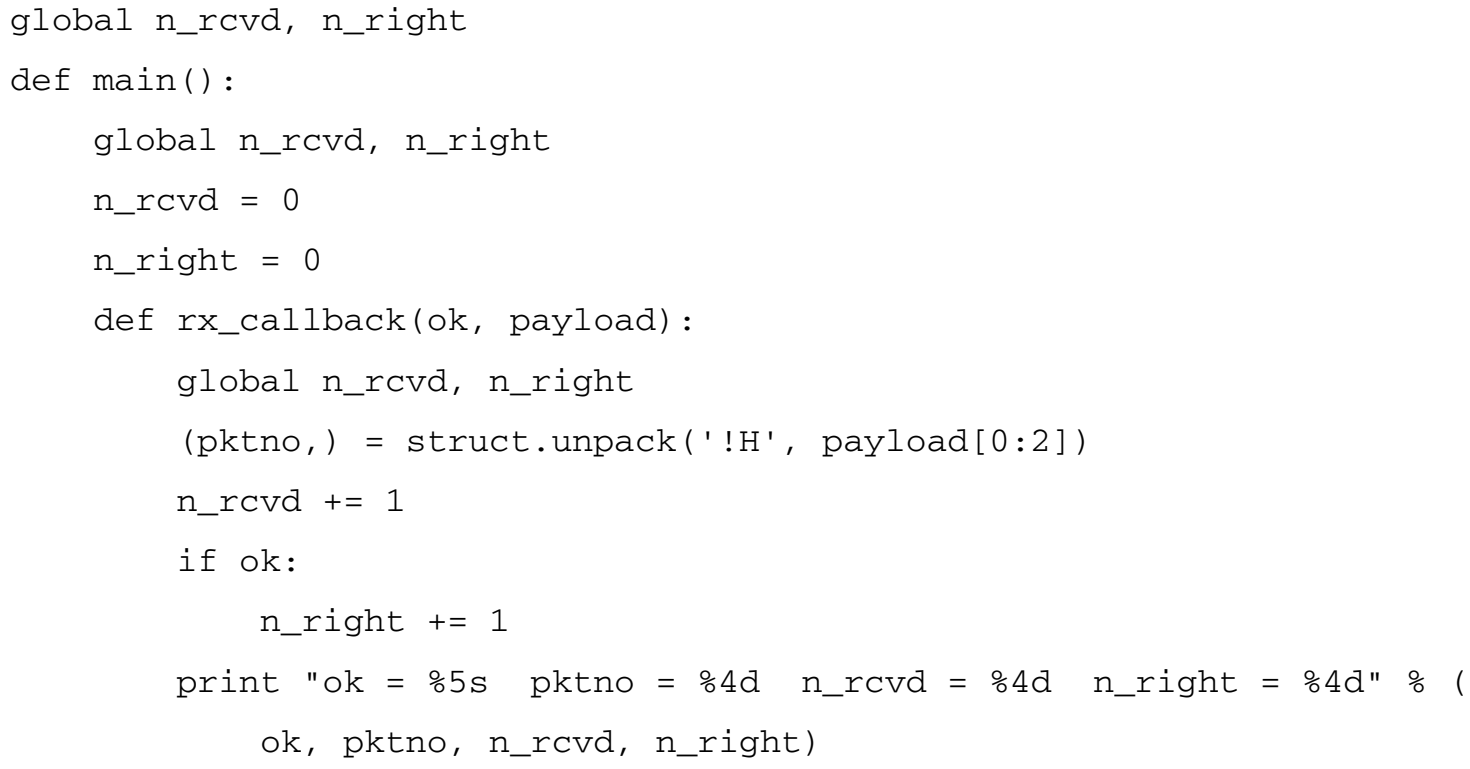

Code example 7: Print packet summary for a receiver packet

\subsubsection{Setup and Perform a Simplex Data transmission}

1. Connect the Basic TX and Basic RX by RF cable (we will not use antenna), see Figure 13. Note that in this figure the RFX2400 is installed (for use in later experiment) - but it is not used in this experiment.

2. Plug the USRP power in (you may need to use an adapted to go from the DC power supply to the local mains power outlet) and connect USB cable to the PC (in this case we are using laptop computer).

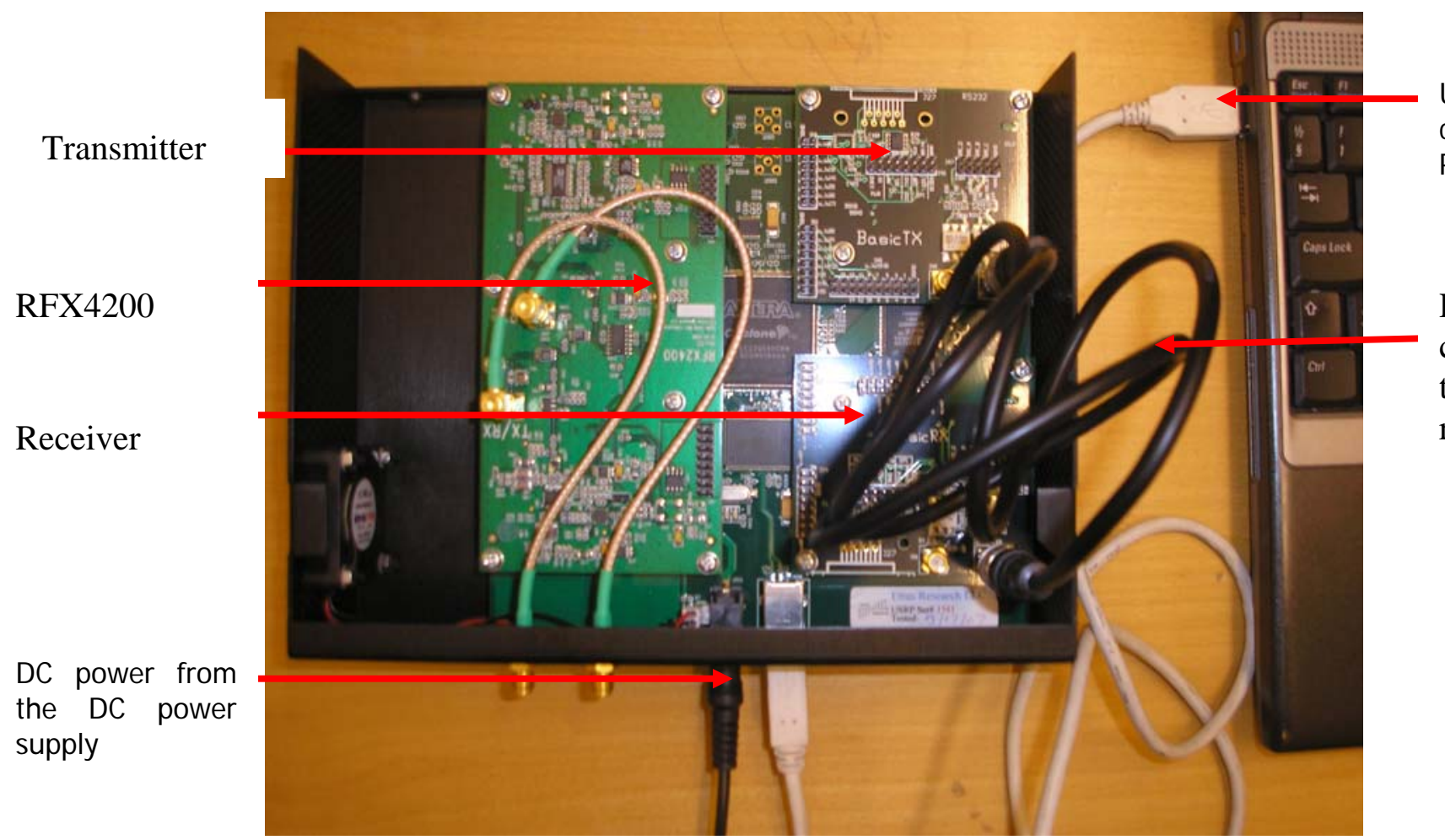

$\mathrm{RF}$

cable

connecting the transmitter and receiver

Figure 13: setup USRP for loopback simplex communication 
3. Open a terminal and start the receiver first. We will use all default values, but specify a $900 \mathrm{MHz}$ frequency.

$$
\text { \$./benchmark_rx.py -f 900M }
$$

4. Open a new terminal and start the transmitter. We will use all default values, but specify $900 \mathrm{MHz}$ frequency.

$$
\text { \$./benchmark_tx.py -f 900M }
$$

Here an example of the output of the transmitter and receiver
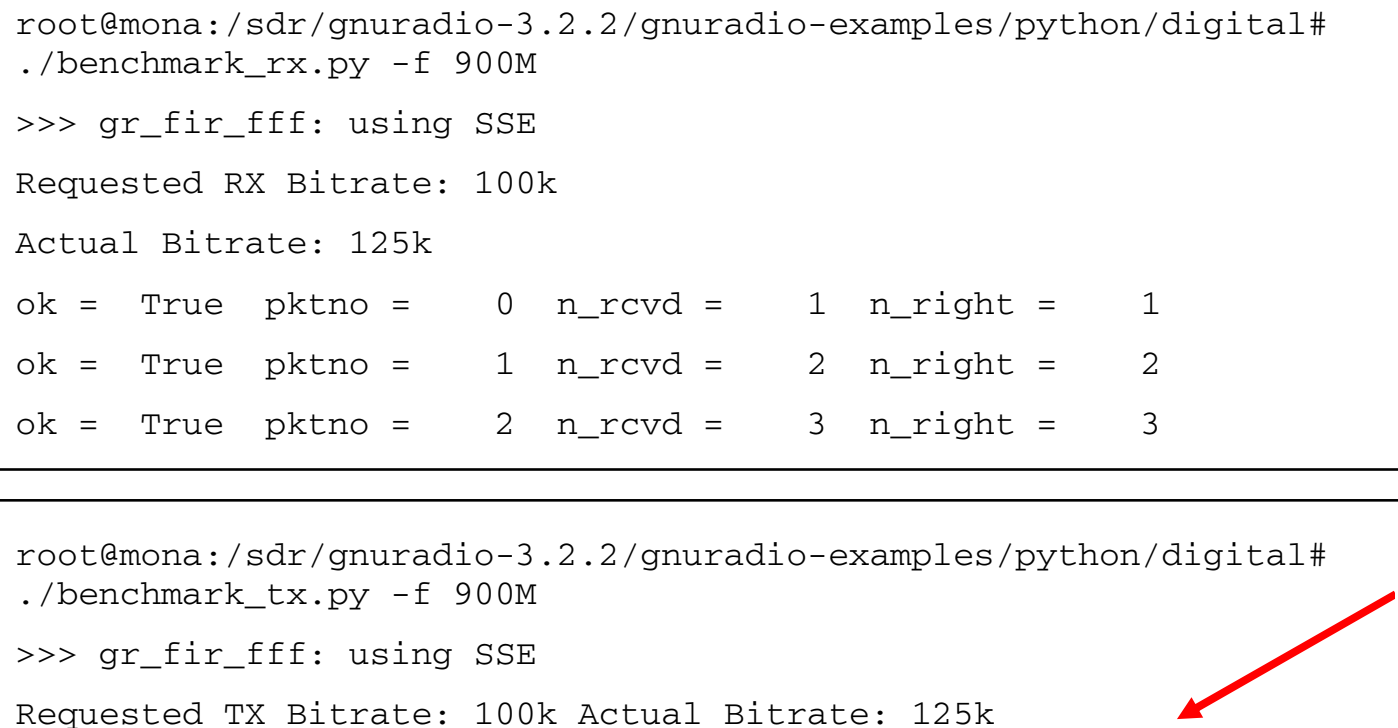

One "." per packet transmitted.

\subsubsection{Student Exercises}

Each student (or group of students) can write lab report cover the following.

1. Calculate the transmission time.

2. Use the other two defined types of modulation. What are the differences between each modulation and how does it affect the transmission?

3. Run the application using two PCs; PCA for the transmitter and PCв for the receiver. And repeat steps 1) and 2). Do you get the same results?

4. What would happen if you used two antennas rather than the RF cable to allow the receiver to listen to the transmission of the transmitter? What frequencies would be emitted? What existing services could this interfere with?

5. [Advanced optional exercise] Use a program such as SnoopyPro to look at the data being set over the USB interface to and from the USRP. What can you learn from examining this traffic? 
6. [Advanced optional exercise] Replace the cable with a "tee" in the middle - connect the tee to an oscilloscope. Look at the resulting signal on the oscilloscope. What do you see when you use different forms of modulation. [Note that a USRP could also be used as an oscilloscope.]

7. [Advanced optional exercise] Replace oscilloscope in the previous exercise with another USRP and use it as a spectrum analyzer. 


\subsection{Experiment 2: Voice Transmission}

This experiment is similar to experiment 1 ; but instead of a file we are sending and receiving a voice signal. The code uses GSM-FR encoder and decoder to as a voice CODEC. see Figure 14 and Figure 15.

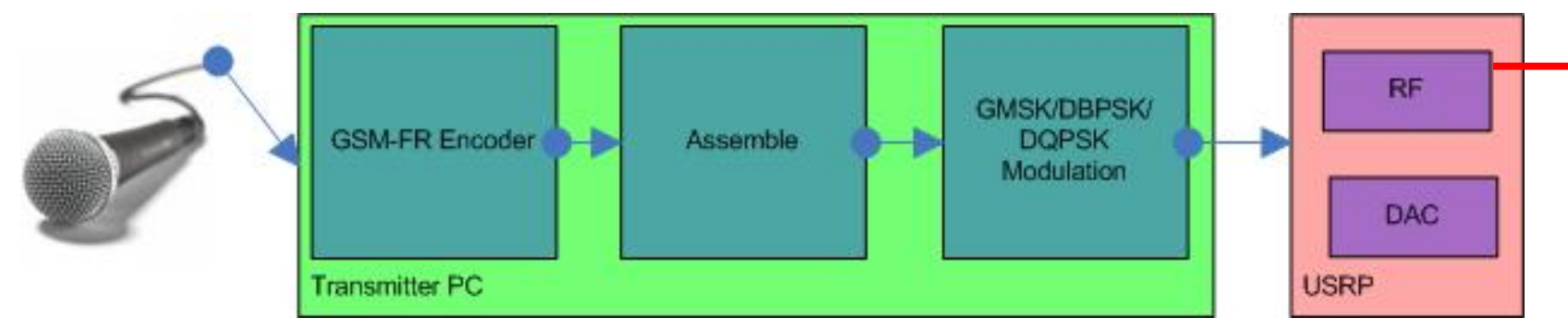

Figure 14: Voice transmitter

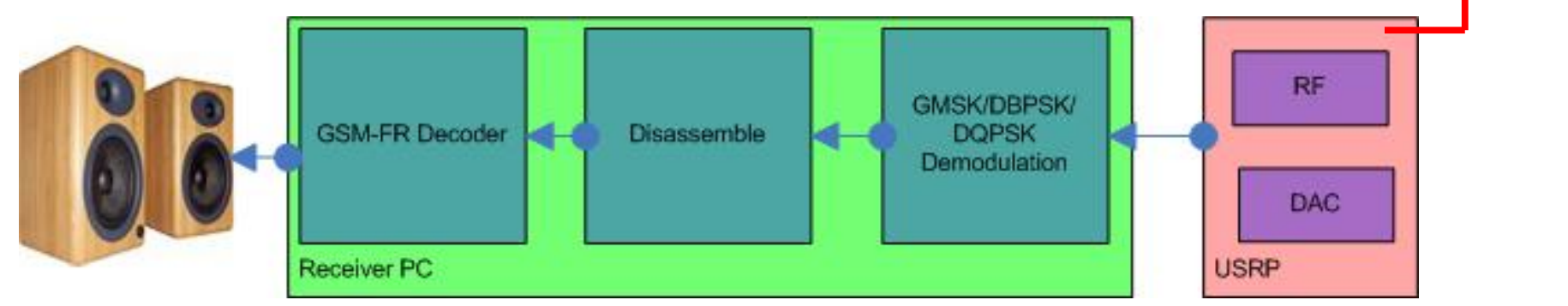

Figure 15: Voice receiver

\subsubsection{Requirements}

- Two USRPs; USRPa with one Basic RX and USRPв one basic TX installed.

- Two PCs; with GNU Radio installed.

- $\quad$ One RF Cable.

\subsubsection{Voice Transmission Code}

The code used in this exercise is part of the GNU Radio examples located in (see Appendix B.3 tx_voice.py and Appendix B.4 rx_voice.py):

/gnuradio-3.2.2/gnuradio-examples/python/digital/tx_voice.py

/gnuradio-3.2.2/gnuradio-examples/python/digital/rx_voice.py

\subsubsection{Setup and Run Voice Transmission}

1. Connect USRP-A Basic RX with USRP-B Basic TX. See Figure 16. 


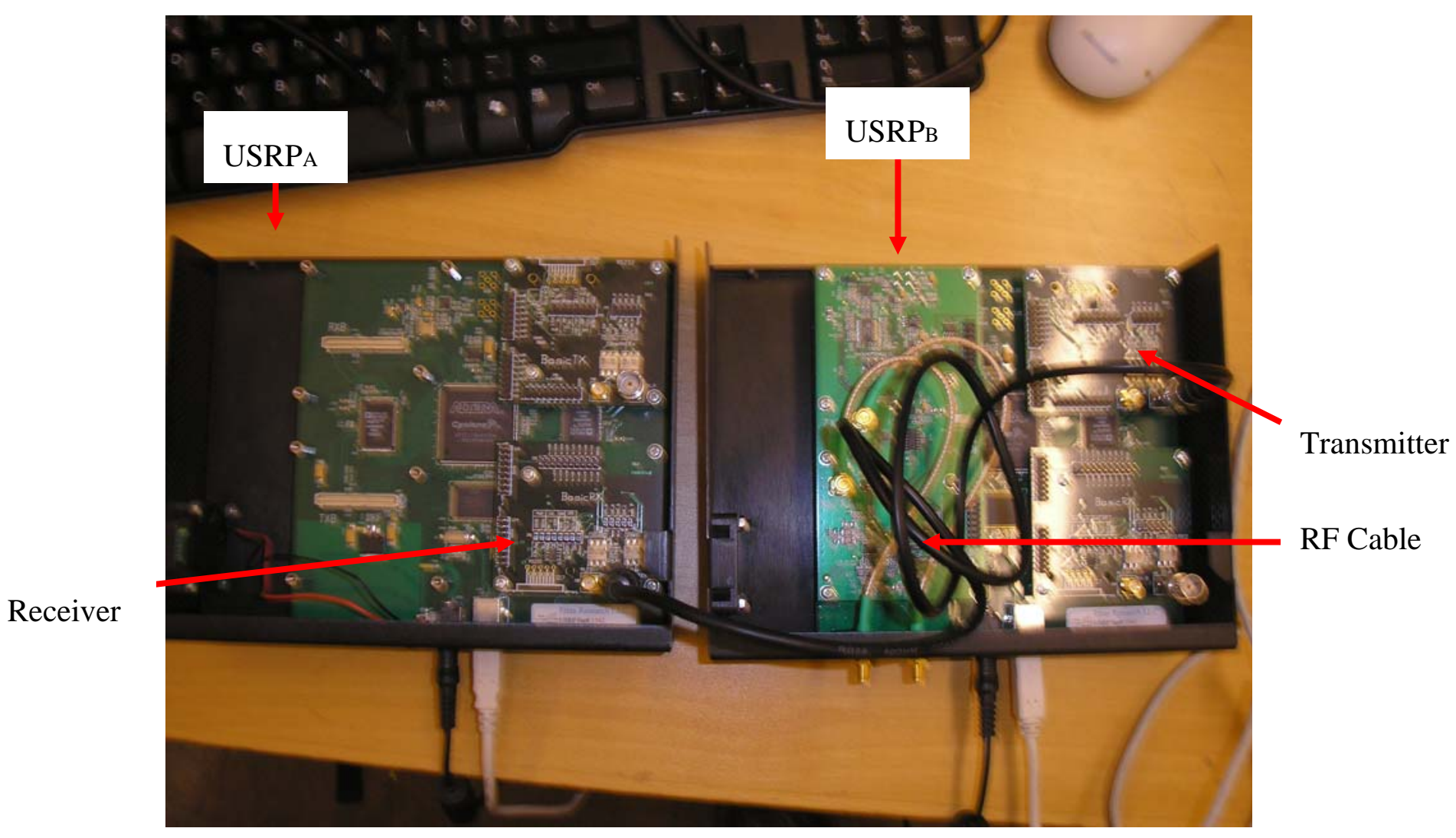

Figure 16: Connecting USRPA Basic RX $\rightarrow$ USRPв Basic TX

2. Connect USRPA with $\mathrm{PC}_{\mathrm{A}}$ and USRPв with $\mathrm{PC}_{\mathrm{B}}$ using USB cable. Make sure that you have connected a speaker to PCA and microphone to РСв.

3. On PCA open a terminal and enter the following command to start the receiver program.

$$
\text { ./rx_voice.py -f 900M }
$$

4. On РСв оpen a terminal and write the following command to start the transmitter program.

$$
\text { ./tx_voice.py -f 900M }
$$

Here an example of the output of the transmitter and receiver 


$$
\begin{aligned}
& \text { \# ./rx_voice.py -f 900M } \\
& \text { >>> gr_fir_fff: using SSE }
\end{aligned}
$$

Requested RX Bitrate: 50k

Actual Bitrate: $125 \mathrm{k}$

gr_buffer::allocate_buffer: warning: tried to allocate

1985 items of size 33. Due to alignment requirements

4096 were allocated. If this isn't OK, consider padding

your structure to a power-of-two bytes.

On this platform, our allocation granularity is 4096 bytes.

$\mathrm{ok}=$ True $\mathrm{n} \_$rcvd $=1 \mathrm{n} \_$right $=1$

aUok $=$ True $n \_r c v d=2$ n_right $=2$

ok $=$ True $n \_r c v d=3$ n_right $=3$

ok $=$ True $n \_$rcvd $=4$ n_right $=4$

aUok $=$ True n_rcvd $=5$ n_right $=5$

"aU" means audio underrun (not enough samples ready to send to

\# ./tx_voice.py -f 900M sound card sink)

>>> gr_fir_fff: using SSE

Requested TX Bitrate: 50k Actual Bitrate: 125k

gr_buffer::allocate_buffer: warning: tried to allocate

1985 items of size 33. Due to alignment requirements

4096 were allocated. If this isn't OK, consider padding

your structure to a power-of-two bytes.

On this platform, our allocation granularity is 4096 bytes.

$\mathrm{uU}$

...uU.

uU

$\mathrm{uU}$

$\mathrm{uU}$

"uU" means underrun (not enough sample ready to send to TTSRP Cinkl

In Appendix B.4 rx_voice.py line 56 the number of messages to hold in the queue specified 33 (you can see that the size of items in the output for both the transmitter and receiver is 33). This information is used by rg_buffer.cc (located in gnuradio-3.2.2/gnuradiocore/src/lib/runtime/) to generate the buffer. The message is a performance warning and it means that the system (i.e. PC) will use more memory and run slower. Code example 1 show part of the gr_buffer which is prints part of the past output samples: 


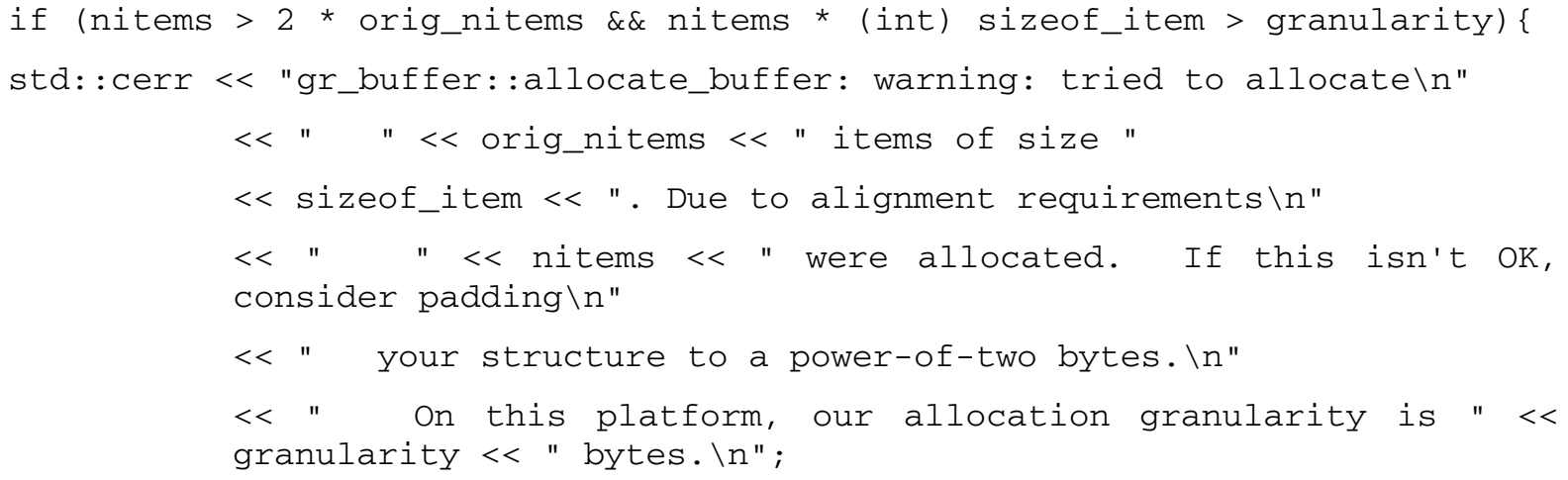

Code example 8: gr_buffer.cc

There is a virtual memory to implement the circular buffer; which is having virtual page mapping to the same physical page. The virtual memory requires first-in-first-out (FIFO) which is an integral number of pages ("items"). Pages are 4096 bytes on x86 and x86-64 machines, the FIFO size is equal of the least common multiple of 4096 and the item size which is one page of 4096 bytes. You can find more information of this circular buffer implementation on [37], search for "how do I disable this buffer warning?".

\subsubsection{Student Exercise}

1. Change the sample rate, decrease it 50 sample per second each time and examine the voice quality. What is the best sample rate you for voice transmission over USRP?

2. Use other modulation and examine the voice quality.

3. When the user is no speaking into the microphone attached to the PC that is acting as the transmitter, what is being transmitter?

4. Does sending voice over the simplex channel differ from sending other data (as in lab experiment \#1)? Why?

5. [Advanced optional exercise] Replace the cable with a "tee" in the middle - connect the tee to an oscilloscope. Look at the resulting signal on the oscilloscope. What do you see when you use different forms of modulation. [Note that a USRP could also be used as an oscilloscope.]

6. [Advanced optional exercise] Replace oscilloscope in the previous exercise with another USRP and use it as a spectrum analyzer. You can use a spectrum analyzer developed by Costa A. J. et. al. [36]. The code of the spectrum analyzer is part of GNU radio and located in: gnuradio/gr-utils/src/python/

7. [Advanced optional exercise] Replace the signal that you look at with using an oscilloscope or spectrum analyzer (in the previous exercises) with the base band voice signal. What can you learn from observing this signal at the transmitter versus this signal as seen at the receiver? What do the characteristics of this signal suggest about how the voice should be encoded and when packets should be transmitted? 


\subsection{Experiment 3: Carrier Sense Multiple Access Protocol}

In this experiment we introduce Carrier Sense Multiple Access (CSMA) (without collision detection) as a link layer protocol. This experiment illustrates a common media access and control protocol (MAC). Its also provides a framework for students to build their own MACs, by modifying the code. In this experiment we will use the "TUN/TAP” Linux interface to intercept frames that are being sent to (or received from) a virtual network interface. This enables the student to run any network protocol or higher level protocol of their choice - while seeng the frames passed to their MAC and physical layer.

TUN/TAP provides virtual network device (in this experiment the device is "gr0") viewed as an Ethernet device. Packets are transmitted and received from or sent to a user space network application. See Figure 17.

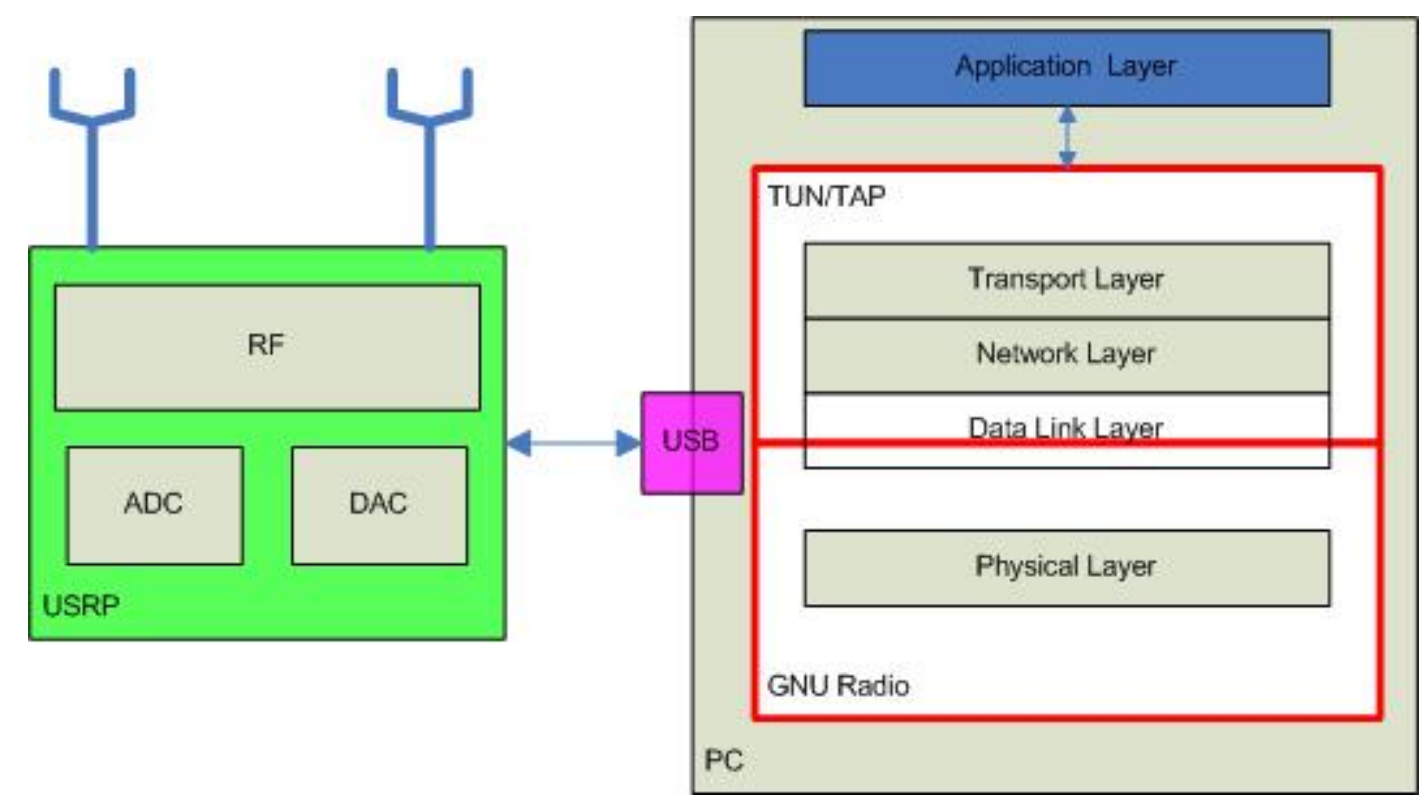

Figure 17: TUN/TAP and GNU Radio

\subsubsection{Requirements}

- Two USRPs; with one Basic RX and one basic TX installed.

- Two PCs; GNU Radio installed.

- Two RF cables

\subsubsection{CSMA code}

The code for this experiment is part of the GNU Radio examples located in (see Appendix B.5 tunnel.py)/gnuradio-3.2.2/gnuradio-examples/python/digital/tunnely.py

The CSMA protocol enables multiple transmitter and receiver to share the same (radio) channel. In CSMA each interface must wait until there is no traffic (this done by listening for the absence of a carrier) on the transmission channel, after the channel is determined to be idle, then the interface can use that channel to send a frame. CSMA can be used together with collision detection (CD) or collision avoidance (CA). However in our case we are using pure CSMA.

The code shown in Code example 9 installs a tap to intercept and deliver frames. For each frame that is received from the tap the code listens for a carrier, if there is a carrier 
present then the code waits for a period of time before listening again. Note that in this code the waiting period is initially 0.050 seconds and this delay is increased in a binary exponential fashion (without limit) until the channel is idle. Each time the channel is sensed, if it is busy the transmitter outputs 'B'.

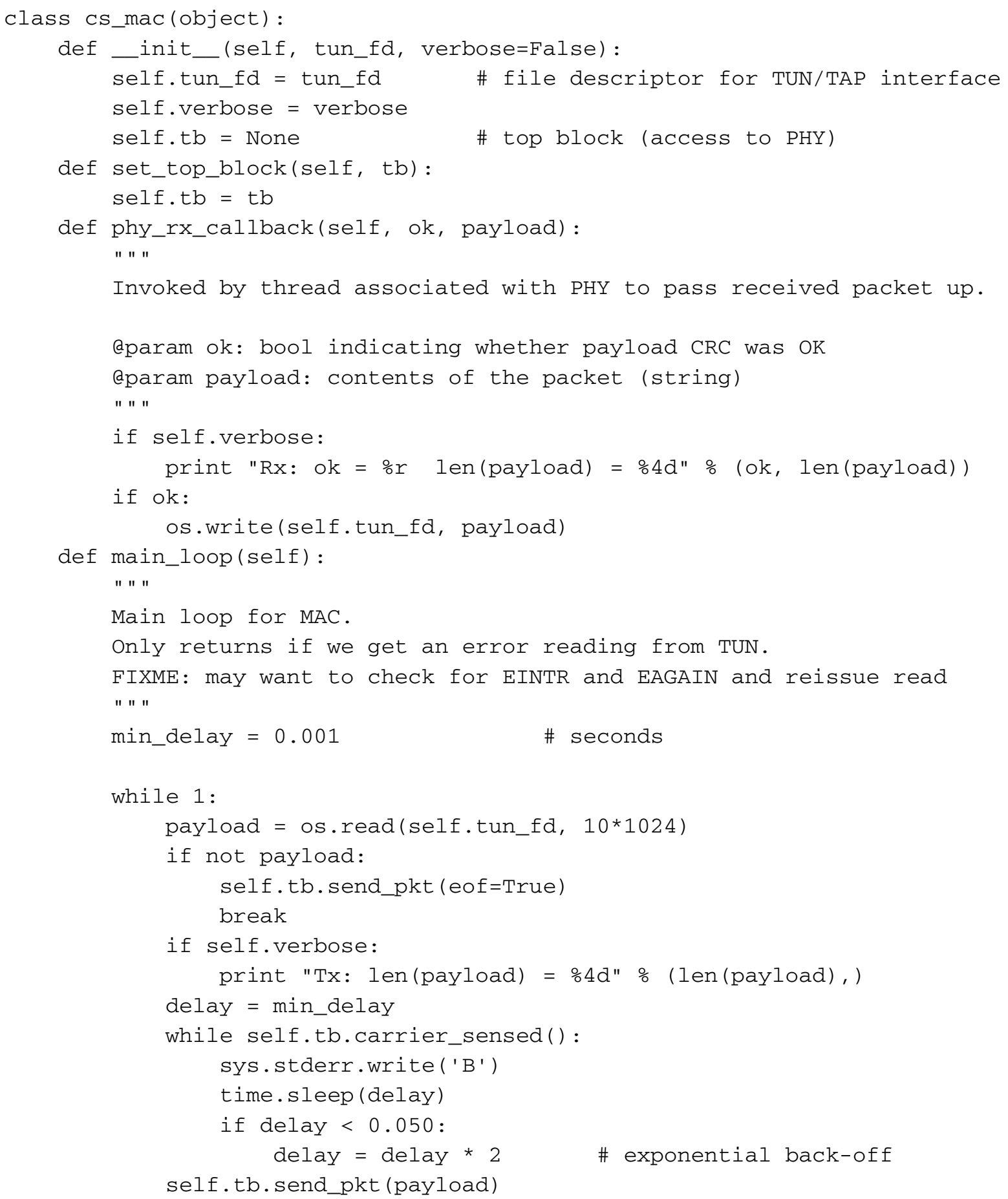

Code example 9: CSMA (transmitter side is is implemented by the main_loop, while the receiver is implemented by the phy_rx_callback) 


\subsubsection{Setup and Run}

1. Connect the two USRP using two RF cables; (see Figure 18)

USRPa Basic TX $\rightarrow$ USRPв Basic RX

USRPA Basic RX $\rightarrow$ USRPB Basic TX

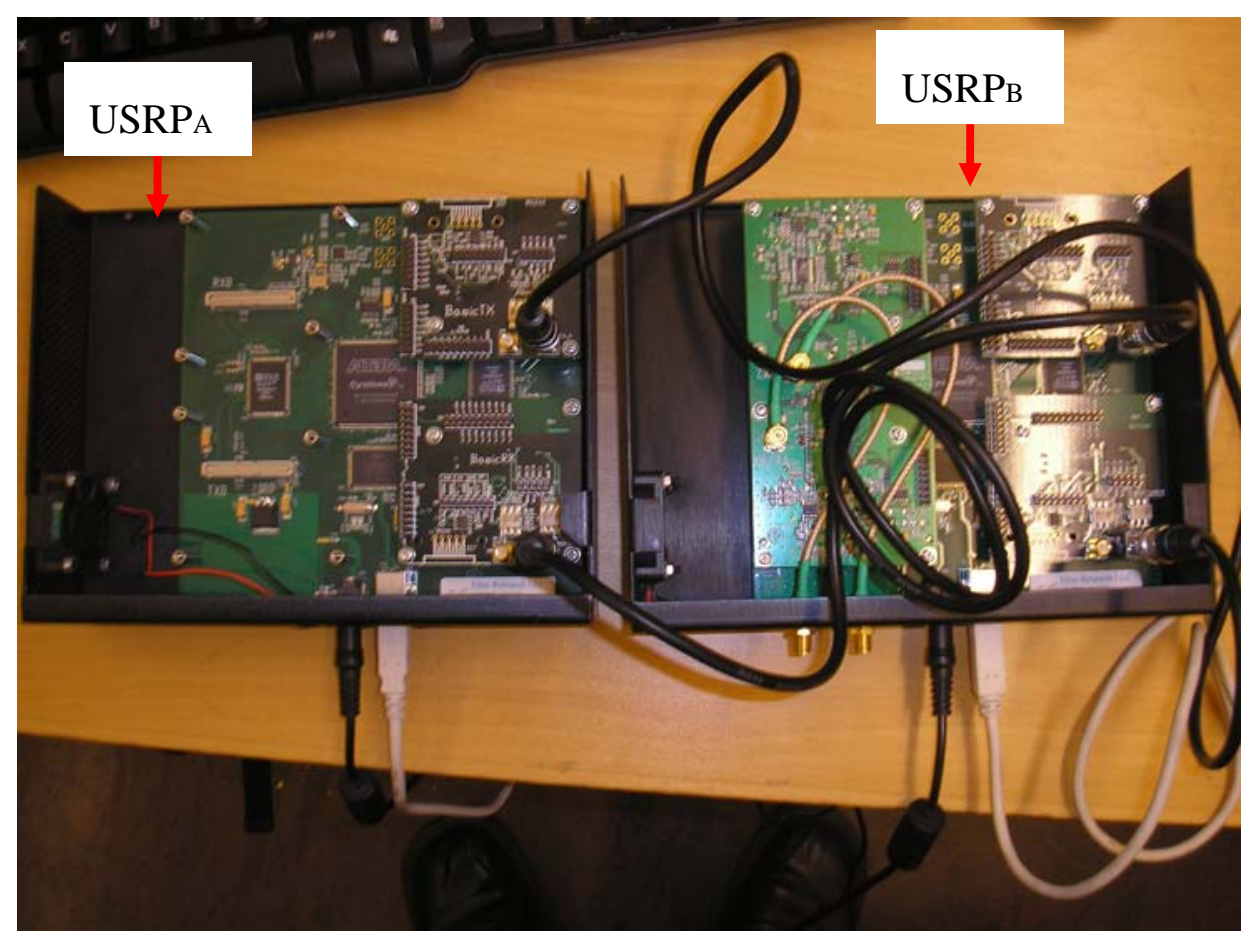

Figure 18: Connecting two USRP

2. On PCA open two terminals

On the first terminal enter the following line which tells the program to use a frequency of $423 \mathrm{MHz}$ with a $500 \mathrm{~K}$ bit per second and output some information for each packet in the terminal's window.

$$
\text { \$./tunnel.py --freq } 423.0 \mathrm{M} \text {--bitrate } 500 \mathrm{k} \text {-v }
$$

In the second terminal write the following line to configure interface gr0

$$
\text { \$ ifconfig gro 192.168.200.1 }
$$

3. On РСв open two terminals

On the first terminal enter the following line which tells the program to use a frequency of $423 \mathrm{MHz}$ with a $500 \mathrm{~K}$ bit per second and output some information for each packet in the terminal's window.

$$
\text { \$./tunnel.py --freq } 423.0 \mathrm{M} \text {--bitrate 500k -v }
$$

In the second terminal write the following line to configure interface gr0

\$ ifconfig gro 192.168 .200 .2 
The following is the output from the first window of the PCA.

\# ./tunnel.py --freq 423.0M --bitrate 500k -v

>>> gr_fir_fff: using SSE

bits per symbol $=1$

Gaussian filter bt $=0.35$

Tx amplitude $\quad 0.25$

modulation: gmsk_mod

bitrate: $\quad 500 \mathrm{~kb} / \mathrm{s}$

samples/symbol: 2

USRP Sink: A: Basic Tx

Requested TX Bitrate: 500k Actual Bitrate: 500k

bits per symbol $=1$

M\&M clock recovery omega $=2.000000$

M\&M clock recovery gain $\mathrm{mu}=0.175000$

M\&M clock recovery $\mathrm{mu}=0.500000$

M\&M clock recovery omega rel. limit $=0.005000$

frequency error $=0.000000$

Receive Path:

modulation: gmsk_demod

bitrate: $\quad 500 \mathrm{~kb} / \mathrm{s}$

samples/symbol: 2

USRP Source: A: Basic Rx

Requested RX Bitrate: 500k

Actual Bitrate: 500k

modulation: gmsk

freq: $\quad 423 \mathrm{M}$

bitrate: $\quad 500 \mathrm{~kb} / \mathrm{sec}$

samples/symbol: 2

Carrier sense threshold: $30 \mathrm{~dB}$

Allocated virtual ethernet interface: gr0

You must now use ifconfig to set its IP address. E.g.,

\$ sudo ifconfig gr0 192.168.200.1 
Be sure to use a different address in the same subnet for each machine.

Tx: len(payload $)=90$

Tx: len $($ payload $)=54$

Tx: len $($ payload $)=153$

Tx: len $($ payload $)=82$

Tx: len $($ payload $)=235$

Rx: ok $=$ False len $($ payload $)=235$

Tx: len $($ payload $)=78$

Tx: len $($ payload $)=235$

\subsubsection{Student Exercises}

1. Open a terminal window on one machine and perform ping command. Look at the delay sending a packet from one machine to the other and back. Compare and analysis your results with Ethernet and IEEE 802.11b network.

2. Capture the traffic using Ethereal and analysis what you got.

3. Modify the code so that you can detect if there is any collision. 


\subsection{Experiment 4: Bluetooth (or IEEE 802.15.4) sniffer}

Bluetooth is low rate low power wireless personal area network solution. Bluetooth devices operate at $2.4 \mathrm{GHz}$ band. The $2.4 \mathrm{GHz}$ band that is used is $83.5 \mathrm{MHz}$ wide (from 2.400 to $2.435 \mathrm{GHz}$ ). This band is divided into 79 channels with a channel spacing of $1 \mathrm{MHz}$. Bluetooth uses spectrum that may be used by other wireless systems (i.e. IEEE 802.11 wireless local area networks, locators (such as used in anti-theft systems in vehicles), cordless telephones, etc.) and may cause interference to other wireless systems as well as receive interference from those other systems. Each Bluetooth device makes 1600 hops per second to implement a fast frequency hopping spread spectrum scheme (at 1/1600 hops per seconds this means that each transmission occurs in a 0.625 millisecond long time slot).

Each Bluetooth device is either a master or a slave. The master Bluetooth device is the device that initiates data exchange and the master Bluetooth device is the device that responds to the master. Both the master and slave devices must use the same sequence of frequency hops to communicate, the master device orders the clock of the piconets, where slaves keeps track of their clocks' offset form the master. In this experiment we will build an application to sniff Bluetooth packets.

It is hard to sniff Bluetooth because of its wide frequency band and fast random hopping (calculated by the master device). We need eleven USRPs to sniff the $83.5 \mathrm{MHz}$ wide band (USRP can work with $8 \mathrm{MHz}$ wide band centred in a frequency), or we can use four USRP2. see

6.2 , 
Table 7 compares between USRP and USRP2.

\subsubsection{Bluetooth Implementation}

In this experiment we will use gr-bluetooth. This code was developed by Dominic Spill and Michael Ossmann [35] and they made the code freely available [26]. In this experiment we will use in which the Bluetooth baseband layer for GNU Radio to implement the Bluetooth baseband processing. In this experiment students will see an example of a SDR. This SDR will be used to listen to packets exchanged between a cellular phone and a Bluetooth headset. Note that Bluetooth uses its own audio coding (using the SBC CODEC), but to listen to the audio requires installing this CODEC.

Bluetooth MAC address is Bluetooth Device Address (BD_ADDR) which is 48 bits comprised of three parts (see Figure 19). Local Area network Profile (LAP) is 24 bits section of the BD_ADDR, Address Portion UAP is 8 bits, and NAP is 16 bits. The NAP and UAP together expresses the company ID which is unique for each Bluetooth device.

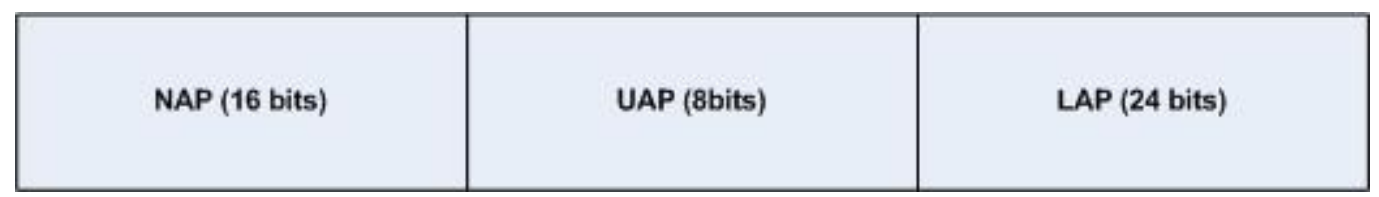

Figure 19: Bluetooth BD_ADDR 


\subsubsection{Equipment}

- One USRP; with one RFX2400 installed (with reverse polarity SMA connector).

- One PC; GNU Radio installed.

- One $2.4 \mathrm{GHz}$ antenna, this antenna we are using has the following specification

$\begin{array}{lcr}\text { Part } & \text { number: } & 30223 \\ \text { Type: } & & \text { whip } \\ \text { Frequency: } & 5 & \mathrm{GHz} \\ \text { Gain: } & \text { Angle: } & \mathrm{dBi} \\ \text { Radiation } & 200 & \mathrm{H} 360^{\circ} / \mathrm{V} 23^{\circ} \\ \text { Range: } & (\mathrm{mm}): & \mathrm{m} \\ \text { Dimensions } & & 197 \times 19 \\ \text { Contact: } & & \text { Rev-SMA } \\ \text { Cable: } & -- \\ \text { Trivia: Multiangle } & \end{array}$

\subsubsection{Installing the system}

The software consists of a signal processing block and a front-end command line tool. The code can be downloaded from the internet site http://sourceforge.net/projects/grbluetooth/. using a web browser, browse to this side and choose "file", then download grbluetooth-0.3.tar.gz, extend Samples and download gr-bluetooth-samples.tar.gz. Next follow the instructions below:

1. Open a terminal window and connect to the directory where you downloaded your files, then enter the following command to unpack and install the code:

\$ tar -xzf gr-bluetooth-0.3.tar.gz

$\$$ cd gr-bluetooth-0.3

\$./configure

$\$$ make

\$ sudo make install

$\$ \mathrm{~cd}$..

2. Copy the file gr-bluetooth-samples.tar.gz to the directory gr-bluetooth/src/python, extract it and rename the output directory to sample. This can be done using the following commands (assuming that you have downloaded the files into the directory /tmp)

\$ cp /tmp/gr-bluetooth-samples.tar.gz gr-bluetooth/src/python gr-bluetoothsamples.tar.gz

$\$$ cd gr-bluetooth/src/python

\$ tar-xzf gr-bluetooth-samples.tar.gz

$\$$ mv gr-bluetooth-samples.tar.gz samples

3. Connect the USRP to your PC. See Figure 20. 


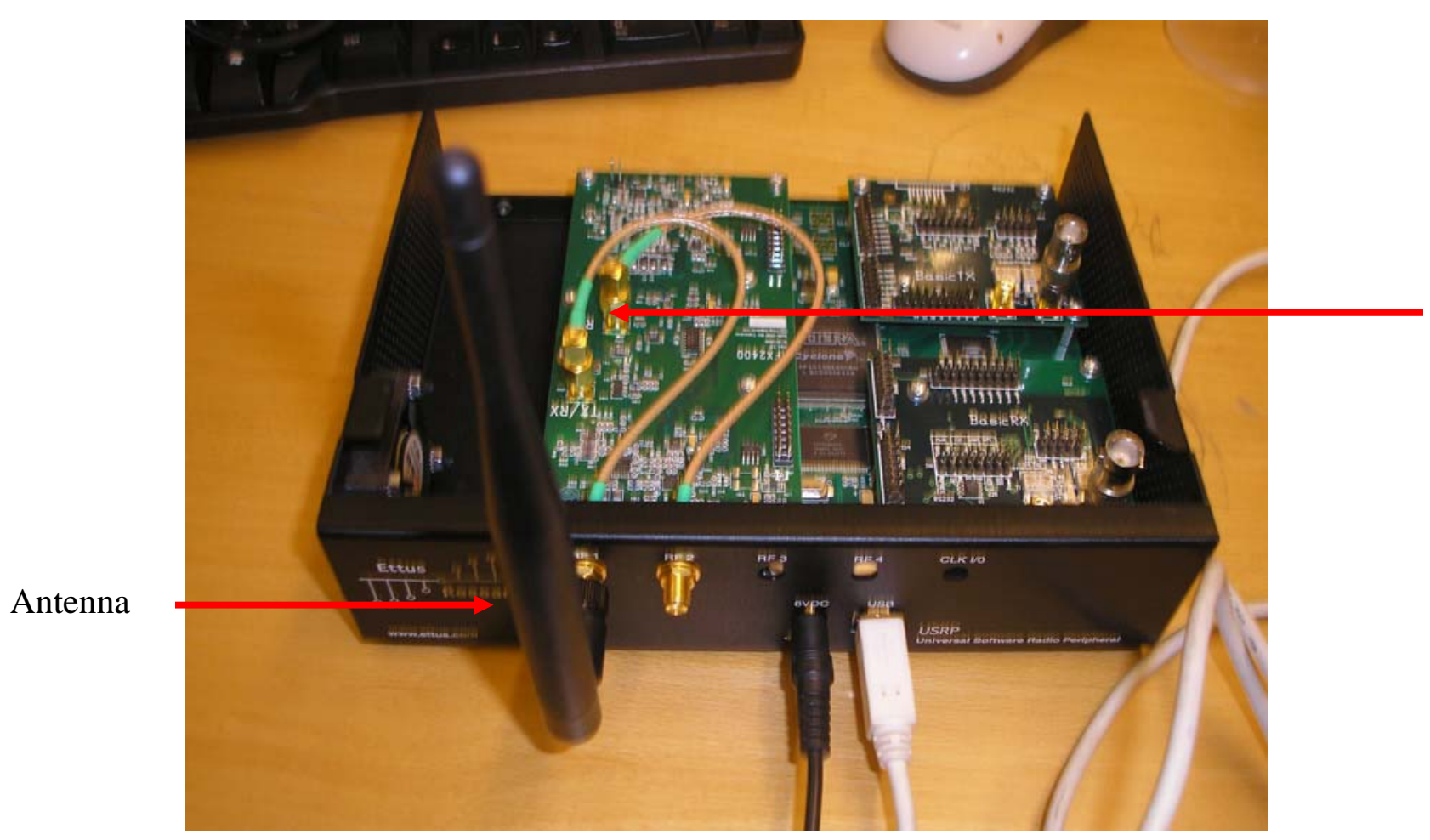

RFX2400

Figure 20: USRP 2.4 GHz Antenna (designed for use with WLAN devices)

\subsubsection{Student Exercise}

The student can carry a report to the instructor includes the solution of this exercises. This exercise uses captured files which are prepared in section 5.4.2 and no need for the USRP to solve the questions. captured files are:

- headset1.cfile: This sample file captured during a call between cell phone and Bluetooth headset at $2.4765 \mathrm{GHz}$ centred frequency with $8 \mathrm{MHz}$ bandwidth using usrp_rx_cfile.py.

- headset3.cfile: This sample file captured during a call between cell phone and Bluetooth headset at $2.476 \mathrm{GHz}$ centred frequency with $2 \mathrm{MHz}$ bandwidth using usrp_rx_cfile.py.

- keyboard1.cfile: This sample file captured during a keyboard typing rapidly plus idle cell phone and headset at $2.4765 \mathrm{GHz}$ centred frequency with $8 \mathrm{MHz}$ bandwidth usrp_rx_cfile.py.

The usrp_rx_cfile.py is part of GNU radio which used to read samples from the USRP and write to file formatted as binary outputs single precision complex float values or complex short values (interleaved 16 bit signed short integers).

This exercise is centred on btrx.py application located on gr-bluetooth/src/python directory. 
Table 6 shows btrx.py options. 
Table 6: btrx.py options.

\section{code Option}

-h $\quad$ Show this help message and exit

-N Number of samples to collect

-R Select USRP Rx side A or B

-S All-piconet sniffer

-a Using a particular aliasing receiver implementation

-c $\quad$ Comma separated list of ddc frequencies

-e $\quad$ Use specified Ethernet interface for USRP2

-d $\quad$ Set fgpa decimation rate to DECIM

-f $\quad$ Set USRP frequency to FREQ

-g $\quad$ Set USRP gain in $\mathrm{dB}$

-i $\quad$ Use named input file instead of USRP

-l $\quad$ LAP of the master device

-m Use USRP2 at specified MAC address

-n Channel number for hop reversal (0-78)

-p Reverse hopping sequence to determine master clock

-r $\quad$ Sample rate of input

-s Input interleaved shorts instead of complex floats

-t $\quad$ Power squelch threshold in $\mathrm{dB}$

$-\mathrm{w} \quad$ Direct output to a tune interface

-2 use USRP2 (or file originating from USRP2) instead of USRP

1. Find packets and display Local Area network Profile (LAP) in headset3.cfile sample file.

2. Discover the Upper Address Portion (UAP) by CRC in keyboard1.cfile sample file.

3. Discover UAP/CLK1-6 by time interval in headset1.cfile sample file.

4. Decode all piconets on all available channels in keyboard1.cfile sample file. 


\subsection{Experiment 5: IEEE 802.11 Implementation}

In this experiment we will use the BBN 802.11 implementation by the Adaptive Dynamic Radio Open-source Intelligent Team and funded by DARPA's ACERT program. This project used GNU Radio and implemented an 802.11 receiver and transmitter [28].

\subsubsection{Requirements}

- One USRP; with one RFX2400 installed (with reverse polarity SMA connector).

- One PC; GNU Radio version 3.1.1 installed.

- One 2.4 GHz antenna, this antenna we are using has the following specification

Part
Type:
Frequency:
Gain:
Radiation
Range:
Dimensions
Contact:
Cable:
Trivia: Multiangle

number:

30223

whip

$2.4 \quad \mathrm{GHz}$

5

Angle:

200

$\mathrm{dBi}$

(mm) :

$\mathrm{H} 360^{\circ} / \mathrm{V} 23^{\circ}$

$197 \times 19$

Rev-SMA

\subsubsection{Installing BBN 802.11}

This section describes how to build BBN 802.11. You will experience a problem installing BBN 802.11 as described in [29] and this release's build guide [25]. The problem is that BBN 802.11 is not longer available with this SVN version. However, you can get the correct code from the BBN80211 - The Comprehensive GNU Radio Archive Network [30]. You will see two versions (i.e. douggeiger for USRP-1 and usrp2_version), get the proper version according to your USRP device (we are using a USRP rather than the newer USRP2). To get the BBN 802.11 code do the following:

svn co https://128.2.212.19/cgran/projects/bbn_80211/branches/ douggeiger/

Before you install their code make sure that you have GNU Radio version 3.1.1 installed on your Linux platform; if you have version 3.2.2 you will receive the following error -- thus it is very important that you install the earlier version of the GNU Radio software. Now you can install the BBN code. If you have not installed the earlier version of the GNU radio code you will experience an error as shown below:

root@ala-laptop:/sdr/bbn/gr-bbn/src/examples\#./bbn_80211b_rx.py -f 2.437G -v -b

Traceback (most recent call last):

File "./bbn_80211b_rx.py", line 126, in <module>

main ()

File "./bbn_80211b_rx.py", line 121, in main

app = app_flow_graph ()

File "./bbn_80211b_rx.py", line 109, in__init_

self.u = usrp_rx(options.decim, options.verbose, options.gain,options.freq) 
File "./bbn_80211b_rx.py", line 57, in _init_

gr.hier_block2._init_(self, "usrp_rx", gr.io_signature(0, 0, 0), gr.io_signature(1, 2, gr.sizeof_gr_complex))

File "/usr/local/lib/python2.5/site-packages/gnuradio/gr/hier_block2.py",

line 42, in __init__

self._hb = hier_block2_swig(name, input_signature, output_signature)

File "/usr/local/lib/python2.5/site-packages/gnuradio/gr/gnuradio_swig_py_runtime.py",

line 995, in hier_block2_swig

return_gnuradio_swig_py_runtime.hier_block2_swig(*args, **kwargs)

RuntimeError: Hierarchical blocks do not yet support arbitrary or

variable numbers of inputs or outputs (usrp_rx)

The problem is that this BBN code was not converted to use the hier_block2 API which is needed for GNU Radio version 3.2.0 and later.

If GNU Radio version 3.2.x I already installed on your machine you have to delete all gnuradio directories and usrp* files from /usr/local/, then install GNU Radio 3.1.1. Finally go to douggeiger (you can change the douggeiger name and for directory organization point of view; we recommend to put BBN 802.11 in the dorectory gnuradio-3.1.1.) and do execute the commands: ./bootstrap \&\& ./configure \&\& make \&\& sudo make install.

\subsubsection{Setup and Implementation}

In this exercise you need one USRP with RFX2400 daughter board installed and 2.4 $\mathrm{GHz}$ antenna; as described in 5.5.2. See Figure 21.

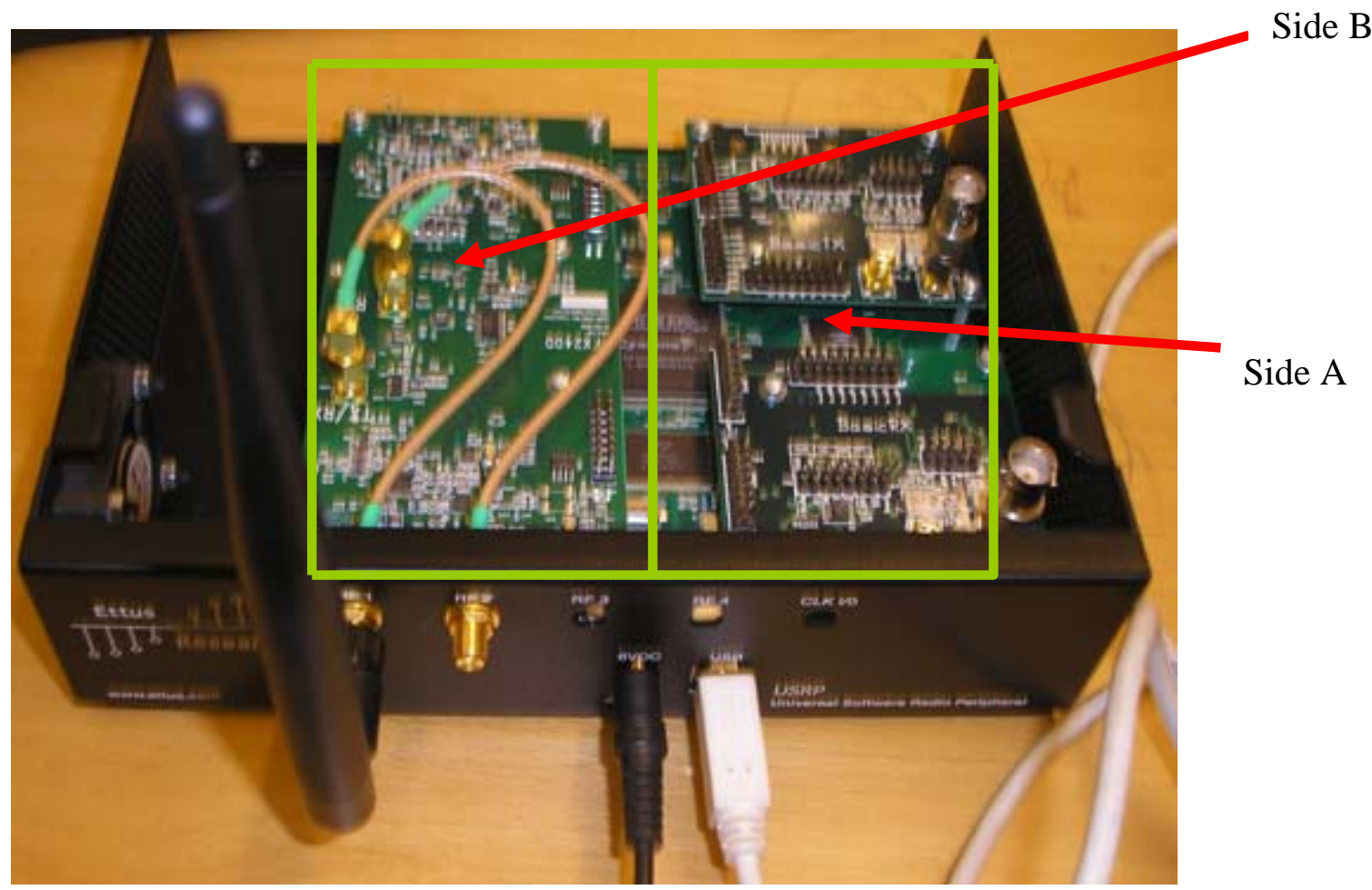

Figure 21: RFX2400, with an Antenna. Note the two sides of the USRP (A and B) 
1. Open a terminal window and connect to the directory gr-bbn/src/examples/, then run the receiver by entring:

./ bbn_80211b_rx.py -R B -f 2.437G -v -b

This will tell the program to use the receiver on "B" (-R B) side, the frequency 2.437 GHZ (-f 2.437G), verbose (-v), and Barker Spreading (-b).

The output will be similar to that shown below.

ala@hlllab2: /gnuradio/gnuradio-3.1.1/adroitgrdevel/gr-bbn/src/examples> bbn_80211b_rx.py -R B -f 2.437G $-\mathrm{v}-\mathrm{b}$

Bits Per Encoded Sample $=8$

adc frequency $=64000000$

decimation frequency $=16$

input_rate $=4000000$

gain $=45.0$

desired freq $=2437000000.0$

baseband frequency 2432000000.0

dxc frequency -5000000.0

Samples per data bit $=8$

>>> gr_fir_ccf: using SSE

gr_vmcircbuf_createfilemapping: createfilemapping is not available

PKT: len=84, rssi $=-43$, src $=00: 1 \mathrm{a}: 70: 3 \mathrm{e}: 4 \mathrm{~F}: 29$, time $=15856$, rate $=1 \mathrm{Mbps}$

PKT: len $=84$, rssi $=-40$, src $=00: 1 \mathrm{a}: 70: 3 e: 4 \mathrm{~F}: 29$, time $=18280$, rate $=1 \mathrm{Mbps}$

PKT: len $=84$, rssi $=-40$, src $=00: 1 \mathrm{a}: 70: 3 e: 4 \mathrm{~F}: 29$, time $=19664$, rate $=1$ Mbps

PKT: len $=84$, rssi $=-43$, src $=00: 1 \mathrm{a}: 70: 3 \mathrm{e}: 4 \mathrm{~F}: 29$, time $=21000$, rate $=1 \mathrm{Mbps}$

PKT: len=84, rssi $=-41$, src $=00: 1 \mathrm{a}: 70: 3 e: 4 \mathrm{~F}: 29$, time $=34456$, rate $=1$ Mbps

2- Open a new terminal and run the transmitter (you will see your frame captured by the receiver) do this:

./ bbn_80211b_tx.py -T B -f 2.437G -b

This will tell the program to use the transmitter on "B" (-T B) side, the frequency 2.437 GHZ (-f 2.437G), and Barker Spreading (-b)

The output will be similar to that shown below.

ala@hlllab2: /gnuradio/gnuradio-3.1.1/adroitgrdevel/gr-bbn/src/examples> bbn_80211b_tx.py -T B -f 2.437G -b

Using TX d'board B: Flex 2400 Tx MIMO B

>> gr_fir_ccf: using SSE

spb: $\quad 8$

interp: 32

The output on the receiver terminal will be similar to that shown below.

ala@hlllab2: /gnuradio/gnuradio-3.1.1/adroitgrdevel/gr-bbn/src/examples> bbn_80211b_rx.py -R B -f 2.437G -v -b 
Bits Per Encoded Sample $=8$

adc frequency $=64000000$

decimation frequency $=16$

input_rate $=4000000$

gain $=45.0$

desired freq $=2437000000.0$

baseband frequency 2432000000.0

dxc frequency -5000000.0

Samples per data bit $=8$

>>> gr_fir_ccf: using SSE

gr_vmcircbuf_createfilemapping: createfilemapping is not available

$\mathrm{uO}$

" $\mathrm{O}$ " means USRP overrun (USRP samples dropped because they weren't read in time.

\subsubsection{Student Exercises}

1. Give examples of how to receive packets from bbn_80211b_rx.py (without dropping them).

2. Look at all sniffed packets and check the rate of each packet. Do you think this system is a full IEEE 802.11b sniffer? Why? 


\section{Evaluation and Analysis}

In this section we will evaluate each of the laboratory experiment from a pedagogical point of view. We should start by noting that these experiments target senior undergraduate student and instructors. The undergraduate student must have studied the following subjects attempting these laboratory experiment s:

1. The student need to have studied at least one high level programming language, preferably object oriented programming language. This will enable the student to understand and the GNU Radio code.

2. Communication systems and computer networks.

3. Signals and systems

4. Digital signal processing.

\subsection{GNU Radio: Analysis}

The GNU Radio provides a extensiv library of signal processing blocks and a glue to tie thises blocks. The radio can be build by creating a flow graph. The signal processing blocks are implemented in $\mathrm{C}++$ programing language, while programers construc the graph and run them in Python.

There is no enghough documentation of how GNU Radio is implemented, during runig application we found that there are some messages printed from diffrernt classes (for example see 5.2.3) and tracing and understanding these message takes some time. The GNU Radio developers did not found acceptable way to provide unifed documentation for the system [38]. However, there is some documentatins for GNU Radio C++ blocks, and you can get help from other developers in [39].

The Gnu Radio has many releases developed. In release version 3.2.x the higher block of the system is updated. This will affect applications developed under old release from running in new releses.

\subsection{USRP: Analysis}

The USRP is a device we used in this thesis to develop undergraduate's experiment . This device has various daughterboards which operate on different radio frequency bands (from DC to $2.9 \mathrm{GHz}$ ); you have to plug-in a sutable daughterboard for you application.

When we are running our applications we experience that closing application using Cotrol+z will not flush the application running process; is you are going to run any application after the one application you will receive a error. We used to unplug the USRP DC power off, and then pulg it in again. Another solution introduced to us is to see all running process, and then "kill” Python process. You can do that by:

ps (to see all running process. find the number of Python application process and enter)

kill $-9<$ Python process number $>$

USRP2 was developed and goes to the market on May 25, 2009. There are some benefits of using USRP2 than USRP, 
Table 7 describes these benefits: 
Table 7: USRP and USRP2 [15]

\begin{tabular}{|l|l|}
\hline USRP & USRP2 \\
\hline $8 \mathrm{MHz}$ instantaneous of RF bandwidth & $25 \mathrm{MHz}$ instantaneous of RF bandwidth \\
\hline $\begin{array}{l}\text { The radio can be accessible from one } \\
\text { computer }\end{array}$ & $\begin{array}{l}\text { the radio to be accessible from more than } \\
\text { one computer }\end{array}$ \\
\hline USB interface & Gigabit Ethernet interfaces \\
\hline Lowest cost & Highest cost \\
\hline Slower FPGA & Faster FPGA \\
\hline ADCs (12-bits $64 \mathrm{MS} / \mathrm{s})$ & ADCs (14-bits $100 \mathrm{MS} / \mathrm{s})$ \\
\hline DACs (140bits $128 \mathrm{MS} / \mathrm{s})$ & DACs (16-bits $400 \mathrm{MS} / \mathrm{s})$ \\
\hline
\end{tabular}

\subsection{Laboratory exercises: Analysis}

The laboratory exercises were designed based upon the idea of step-by-step learning. The undergraduate student initialy follow the steps presented in each experiment to solve a problem and understands subject terms. These experiments start with simple communication systems first, a little bit complex systems, and finaly real world systems. In each experiment, the student must solve specific problems and submit a written report to the instructor. The instructor can choose which experiment are sutable for the students.

Experiment 1: simplex data communication, in a simple application to data transmission. In this initial experiment, the student can exmine in detalis how the three lower layers of the OSI model are implemented and different methods of modulation can be used - while supporting the same higher level protocol. The student can develop a ptotocol in any packet format, and can use the code represented for this exprimet to develop a feedback from the receiver.

Experiment 2 shows the student encoded voice data can be transmitted over a digital channel. In the exercises the student is ask to think about what would happen if the cable were replaced with a pair of antennas and the RF signal were to be transmitted and transmitt on the air.

Experiment 3 exposed the student to a spesific MAC layer protocol. The student see how he or she might can bulid his or her own MAC protocol A central element of this experiment is that the MAC protocol simply implements a protocol. (An optional exercise for this experiment would be to ask the student to write the protocol specification that is actually implemented by the code.). It is difficult for the student to implemetnt CSMA/with collition detection, because of the anttena power limitation. However, the student can implement CSMA/with collition avoidance.

Experiment 4 takes students deep to Bluetooth protocol. This experiment illustrates some very sophisticated aspects of protocol analysis and has some important observations for student's about the lack of security through obscurity (specifically that fast frequency hopping and not putting the complete MAC address in Bluetooth frames does not prevent someone from listing to these packets nor does it hide the devices), and let bluetooth works. 
Experiment 5 tells the student how to implement 802.11 protocol. This experiment is sutable only for last year undergraduate students. Moreover we can use it only with IEEE 802.11 and not IEEE 802.11b because of the limitation of USB2 transmission. 


\section{Conclusions and suggested future work}

We developed laboratory experiment for undergraduate students to help them understands media and access control protocols protocol. The experiments are designed in a way that easy to understand experiments first, and the complicated experiments. Instructors might use these experiments and add more exercises to develop their own lessons plan and course material.

In this thesis we present software defined radio application built on USRP and GNU Radio. Thus, our first goal was achieved. However, we did not develop our own application using USRP and GNU Radio, which is goal two. If we look at the laboratory experiments we can see that it includes different kind of applications, in which we spend our time. But if this thesis was designed to build specific application using USRP and GNU Radio, then we can spend our time on single application. Moreover, the development time for applications using USRP and GNU Radio is varied form application to other. For example, Bluetooth (or IEEE 802.15.4) sniffer developed by two developers and they spends three months to make it running.

In conclusion, we can say that it is not easy job to implement applications using USRP and GNU Radio because of the weak documentation of the GNU Radio. And if we started this thesis again we would develop a documentations tool for GNU Radio to help developers to implement their own applications.

The computer science department of Grove City College, has developed some exercises based on SDR for undergraduate projects [13]. These exercises enable students to receive real-time waveforms; specifically to receive AM, FM, and SSB signals. They are reported to be developing a plug-in for commercial radio broadcasts in which an AM radio will have the current FM station quality and the quality of broadcast FM stations will be CD quality.

Compared to our solution, the Grove City College research targets broadcast radio, while we focus on wireless local area networks and personal area networks.

\subsection{Future work}

1) Create experiments based on GNU Radio Companion (a graphical tool for creating signal graph to generate flow graph source code) [31].

2) Create experiments base simulink [32].

3) Create experiment to listen to a GSM cell phone [33].

4) Create experiment for ZigBee.

5) Create fully a receiver experiment for $802.11 \mathrm{~b}$ 


\section{References}

[1] Susan Karlin,’Tools \& Toys: Hardware for your Software Radio”, IEEE Spectrum, 34(10) , Oct. 2006, pp51-54.

[2] Paul Burns, ”Software Defined Radio for 3G”. London. Artech House, 2003.

[3] Bruce A. Fette, et al, ”Cognitive Radio Technology” Newnes, 2006, 656 pages, ISBN-10: 0750679522, ISBN-13: 978-0750679527.

[4] T.W. Parks and J.J. McClellan, "Chebyshev Approximation for Nonrecursive Digital Filters with Linear Phase,” IEEE Transactions on Circuit Theory, Vol. 19, 1972, pp. 189-194.

[5] L.R. Rabiner, J.H. McClellan, and T.W. Parks, "FIR Digital Filter Design Techniques Using Weighted Chebyshev Approximations,” Proceedings of the IEEE, Vol. 63, 1975, pp. 595-610.

[6] Walter Tuttlebee, et al, ”Software Defined Radio: Enabling Technology”, USA, John Wiley \& Sons Ltd, 2002.

[7] John Bard and Vincent J. Kovarik Jr., "Software Defined Radio: the software communication Architecture”, John Wiley \& Sons Ltd, 2007.

[8] GNU Radio, web page” www.gnuradio.org “, visited 2009-02-10

[9] F. Ge, Q. Chen, Y. Wang, T. W. Rondeau, B. Le, and C. W. Bostian, "Cognitive Radio: From Spectrum Sharing to Adaptive Learning and Reconfiguration," in IEEE Aerospace Conference. Big Sky Montana, MT, March 2008.

[10] Python Programming Language, web page “ www.python.org”, visited 2009-02-10.

[11] Peter Norton, Alex Samuel, David Aitel, Eric Foster-Johnson, Leonard Richardson, Jason Diamond, Aleatha Parker, and Michael Roberts, Beginning Python, Canada, Wiley Publishing, Inc, 2005.

[12] SDR Forum, Web site “www.sdrforum.org ”, visited 2009-02-10.

[13] William Birmingham and Leah Acker, "Software-defined radio for undergraduate projects”, ACM, session:Embded systems and architecture, Volume 39, Issue 1, March 2007, pp. 293 - 297, ISSN:0097-8418.

[14] Open Source SCA Implementation - Embedded, web page “http://ossie.wireless.vt.edu/index.html”, visited 2009-02-11.

[15] Ettus Research LLC, web page ” www.ettus.com”, visited 2009-11-11.

[16] General Dynamics C4 Systems, Web site ” www.gdc4s.com” visited 2009-07-01.

[17] Analog Design, AD9862 12-/14-Bit Mixed Signal Front-End (MxFE®) Processor for Broadband Communications, Data Sheet, Revision 0, Dec. 2002, internet site "http://www.analog.com/static/imported-files/data_sheets/AD9860_9862.pdf" 
[18] IEEE, Standard Hardware Description Language Based on the Verilog ${ }^{\circledR}$ Hardware Description Language -Description, IEEE Std 1364-1995, IEEE, Oct. 1996, ISBN: 1-55937-727-5, E-ISBN: 0-7381-3065-6.

[19] IEEE SystemVerlog Working Group, IEEE 1800, Web Site ” http://www.eda.org/svieee1800/”.

[20] Deepak Kumar Tala, Verilog Tutorial, web page, Jan. 10, 2009 http://www.asicworld.com/verilog/veritut.html.

[21] Eric Blossom, "Exploring GNU Radio: Tools fo Exploring the RF spectrum", Linux Journal, Issue 122, June 2004.

[22] Eric Blossom, How to Write a Signal Processing Block, Web page, Jul 21,2006, http://www.gnu.org/software/gnuradio/doc/howto-write-a-block.html.

[23] Greg Colvin and Beman Dawes, Smart Pointers, Web Page, March 11, 2009, http://www.boost.org/libs/smart_ptr/smart_ptr.htm.

[24] Joseph Mitola III,web page, Nov 21, 2008 ”http://web.it.kth.se/ maguire/jmitola/”.

[25] Build Guide- GNU Radio, web page,2009-11-05 ” http://gnuradio.org/trac/wiki/BuildGuide”.

[26] Gr-Bluetooth, web page, Aug 18, 2009,” http://sourceforge.net/projects/grbluetooth/”

[27] “Wireless an Mobile Network Architecture”, G.Q.Maguire Jr. <maguire@kth.se>, http://www.it.kth.se/courses/IK2555/Coursepage-Spring-2010.html

[28] Troxel Gregory D, Blossom Eric, et al “Adaptive Dynamic Radio Open-source Intelligent Team (ADROIT): Cognitively-controlled Collaboration among SDR Nodes”, Networking Technologies for Software Defined Radio Networks, 2006. SDR '06.1st IEEE Workshop, Sep 2006, pp 8-17, ISBN: 1-4244-0733-8.

[29] Other Code - GNU Radio, web page, "http://gnuradio.org/trac/wiki/OtherCode”, visited Nov 7, 2009.

[30] BBN80211 - The Comprehensive GNU Radio Archive Network, web page, “https://128.2.212.19/wiki/BBN80211”, visited Nov 7, 2009.

[31] GNU Radio Companion, web page, “http://gnuradio.org/trac/wiki/GNURadioCompanion”, visited Nov 7,2009.

[32] Simulink-USRP: Universal Software Radio Peripheral (USRP) Blockset, web page, visited Nov 7, 2009.

The NetBSD Packages Collection, web page, "http://ftp.sunet.se/pub/NetBSD/packages/pkgsrc/ham/gnuradiogsm/README.html”, visited Nov 7,2009. 
[34] Alister Burr, "Modulation and coding: for wireless communications", Prentice Hall/Pearson Education, 2001, ISBN: 0201398575.

[35] Michael Ossmann and Dominic Spill, "Building an All-Challe Bluetooth Monitor", ShmooCon 2009, 6 February 2009.

[36] Costa A. j. et. al., "Spectrum analyzer with USRP, GNU Radio and MATLAB”, 7th Conference on Telecommunication, Portugal, May 2009.

[37] Discuss-Gnuradio Archives, web page, "http://lists.gnu.org/archive/html/discussgnuradio/”.

[38] GNU Radio 3.2svn C++ API Documentation, web page, "http://gnuradio.org/doc/doxygen/index.html”, May 22, 2009. 


\section{Appendix A: gr_block.h}

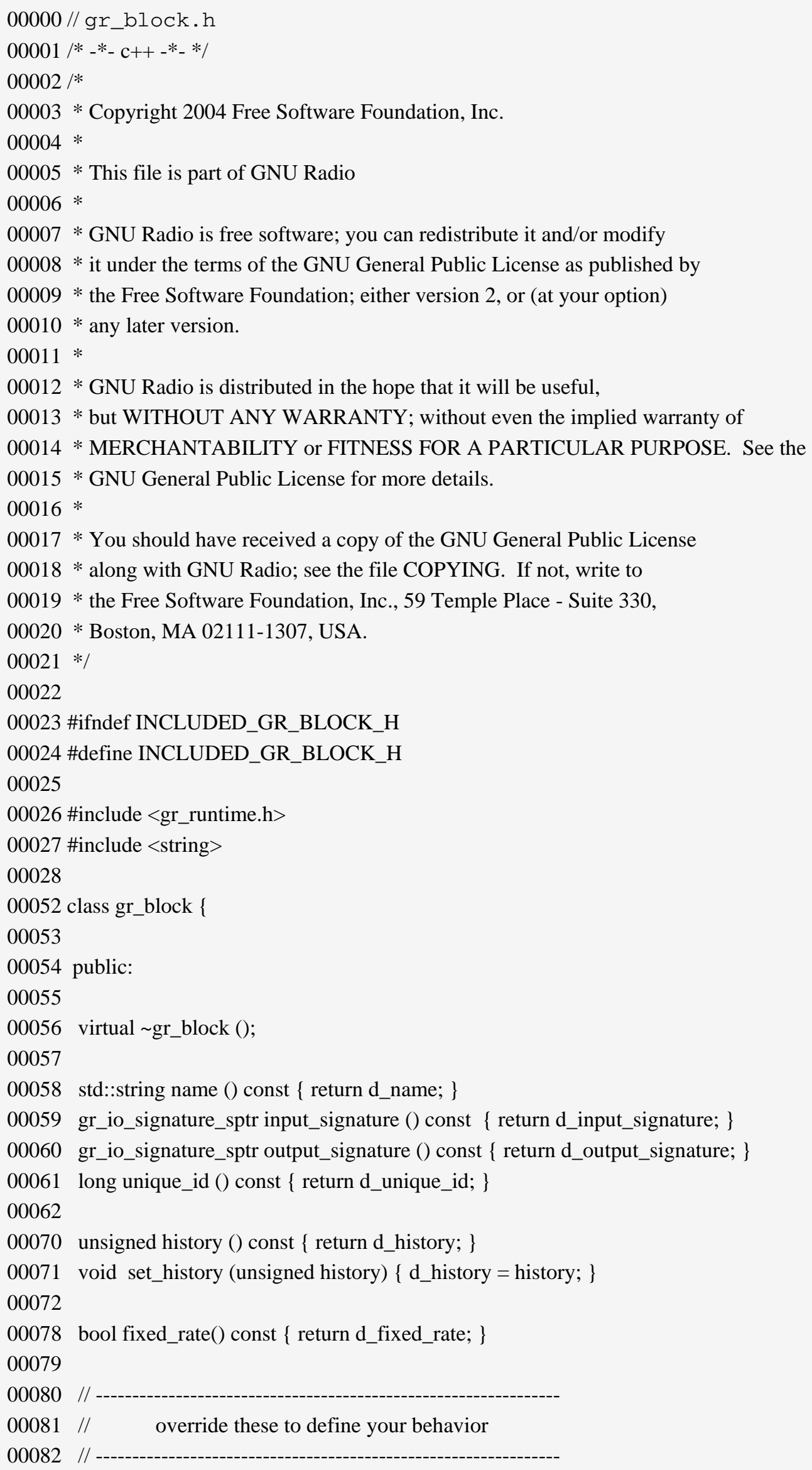




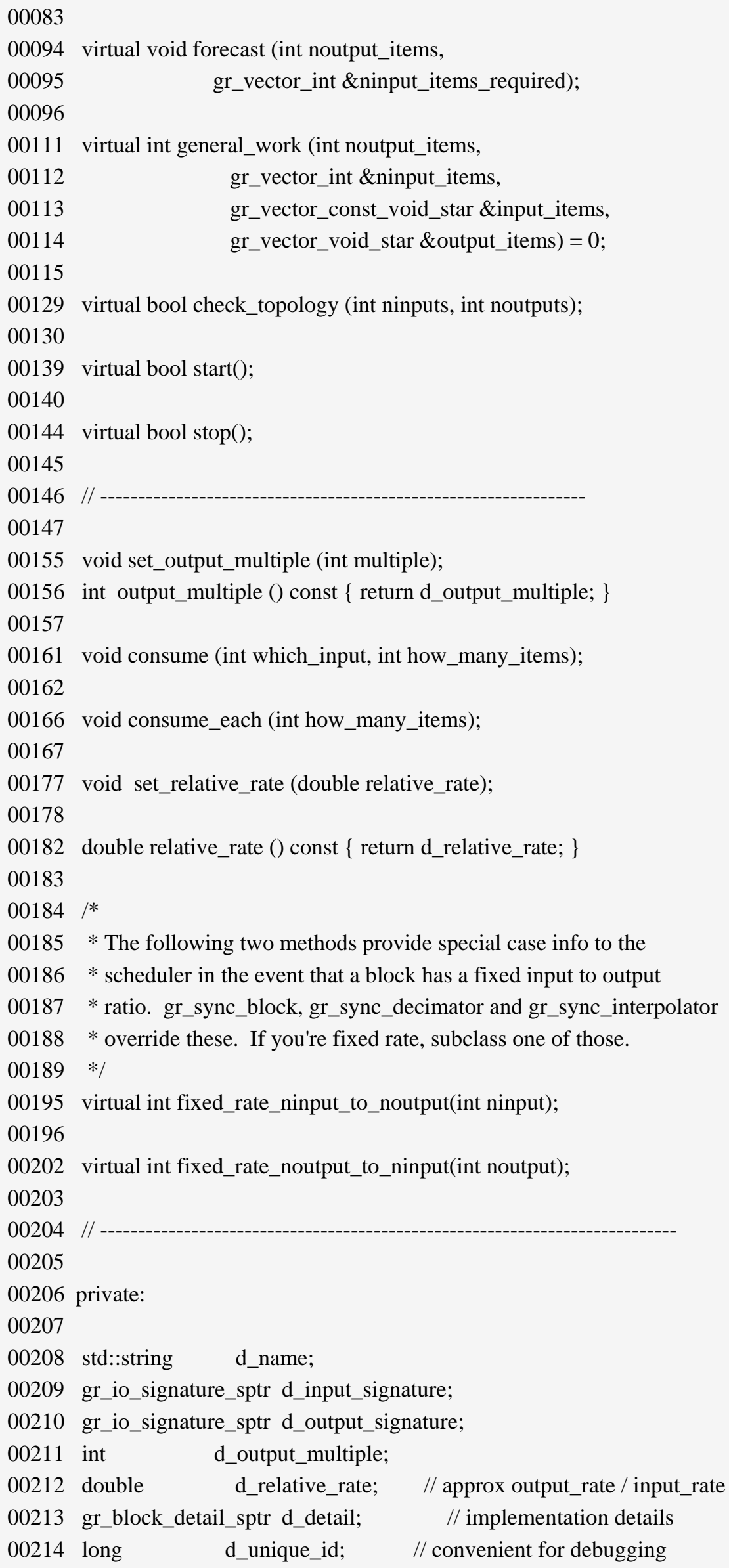




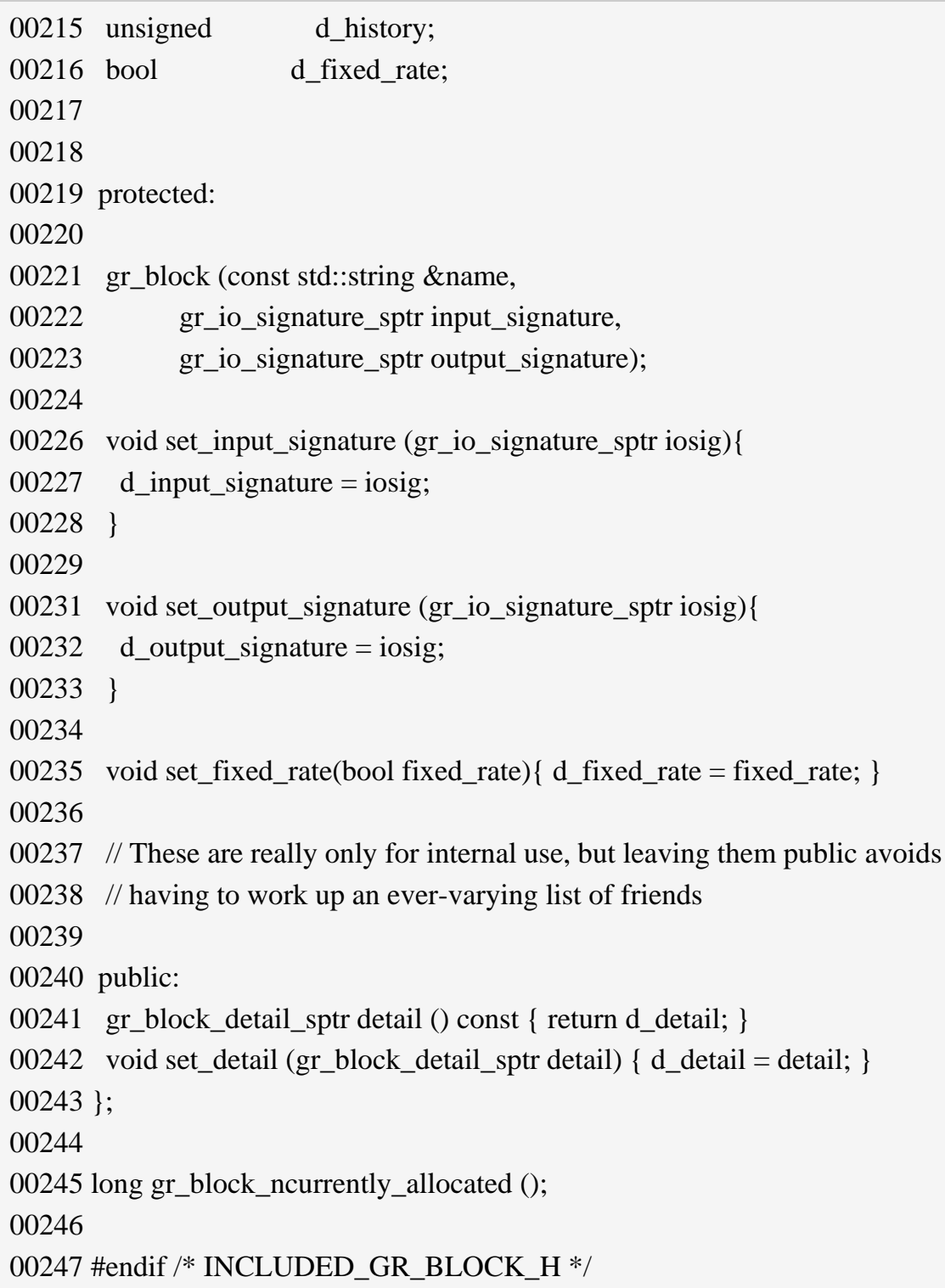




\section{Appendix B: Laboratory Experiments}

\section{Appendix B.1 benchmark_tx.py}

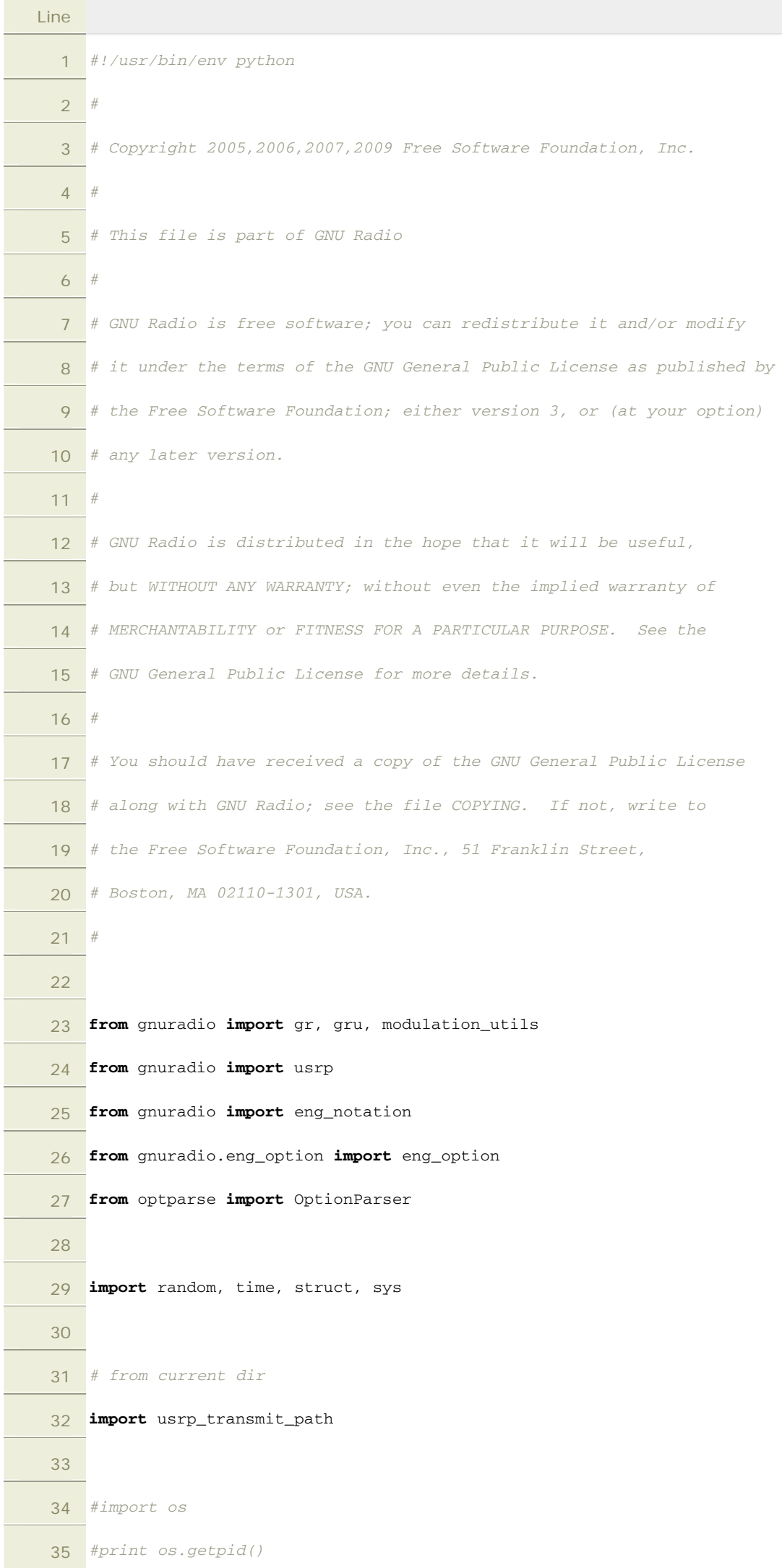




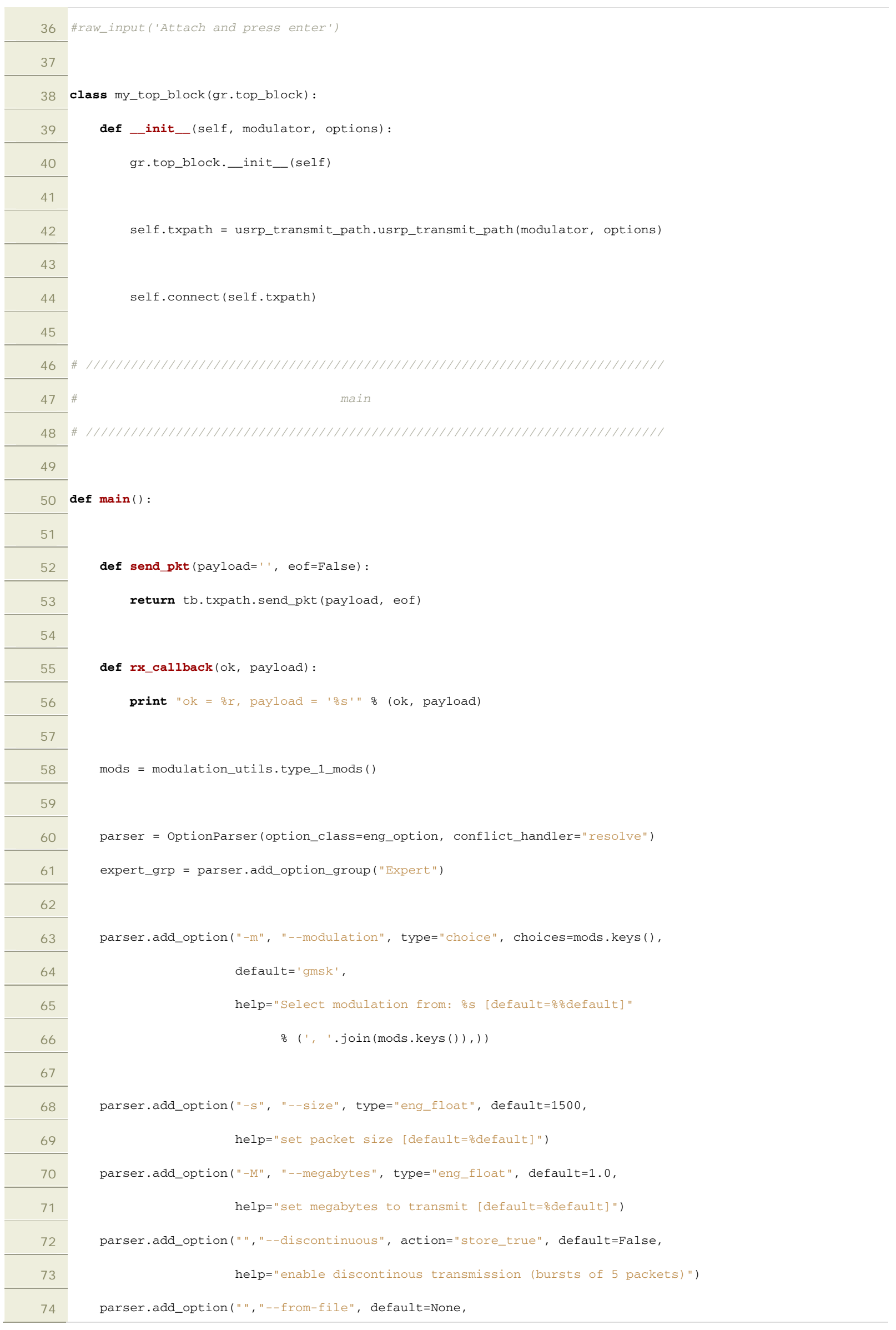




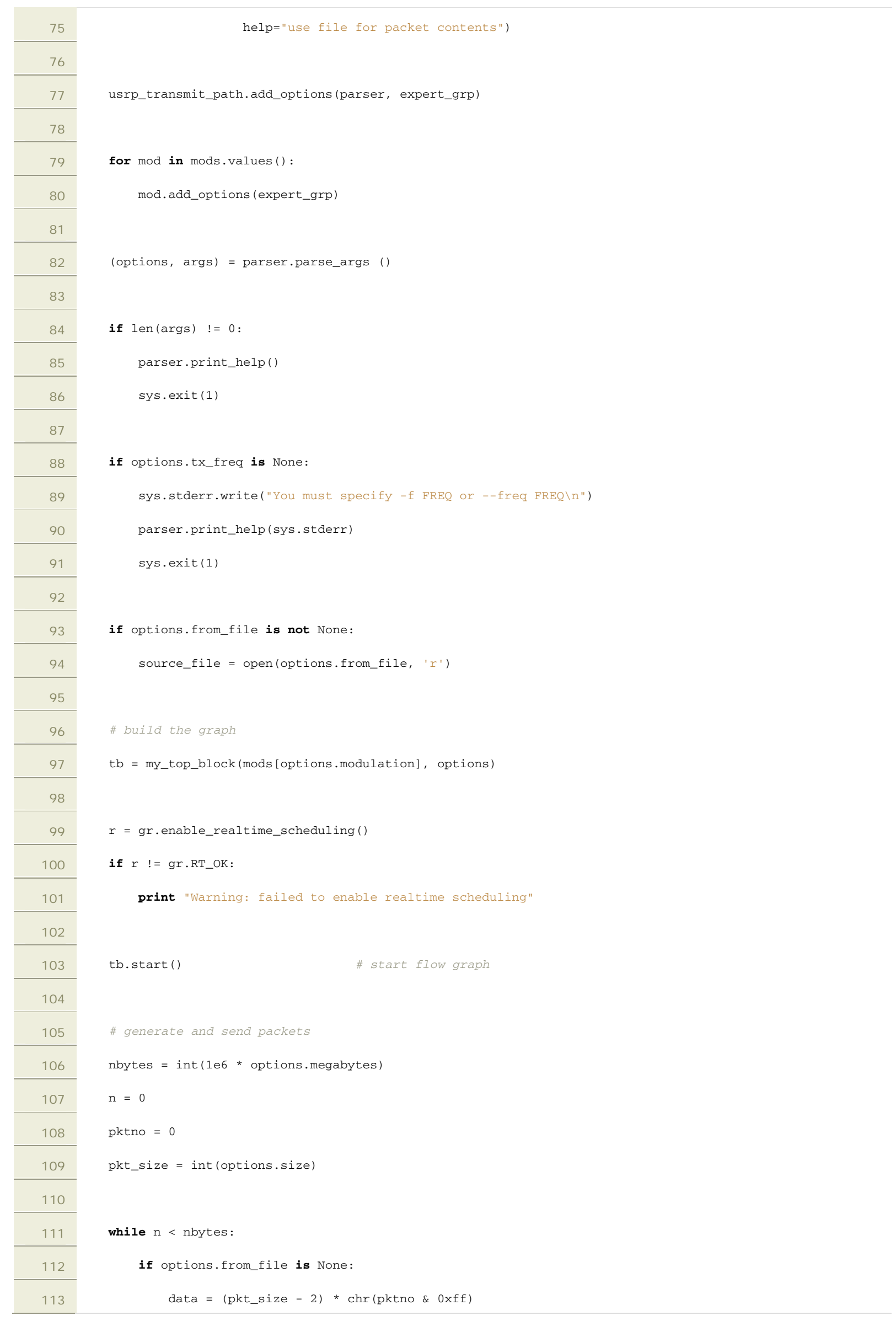




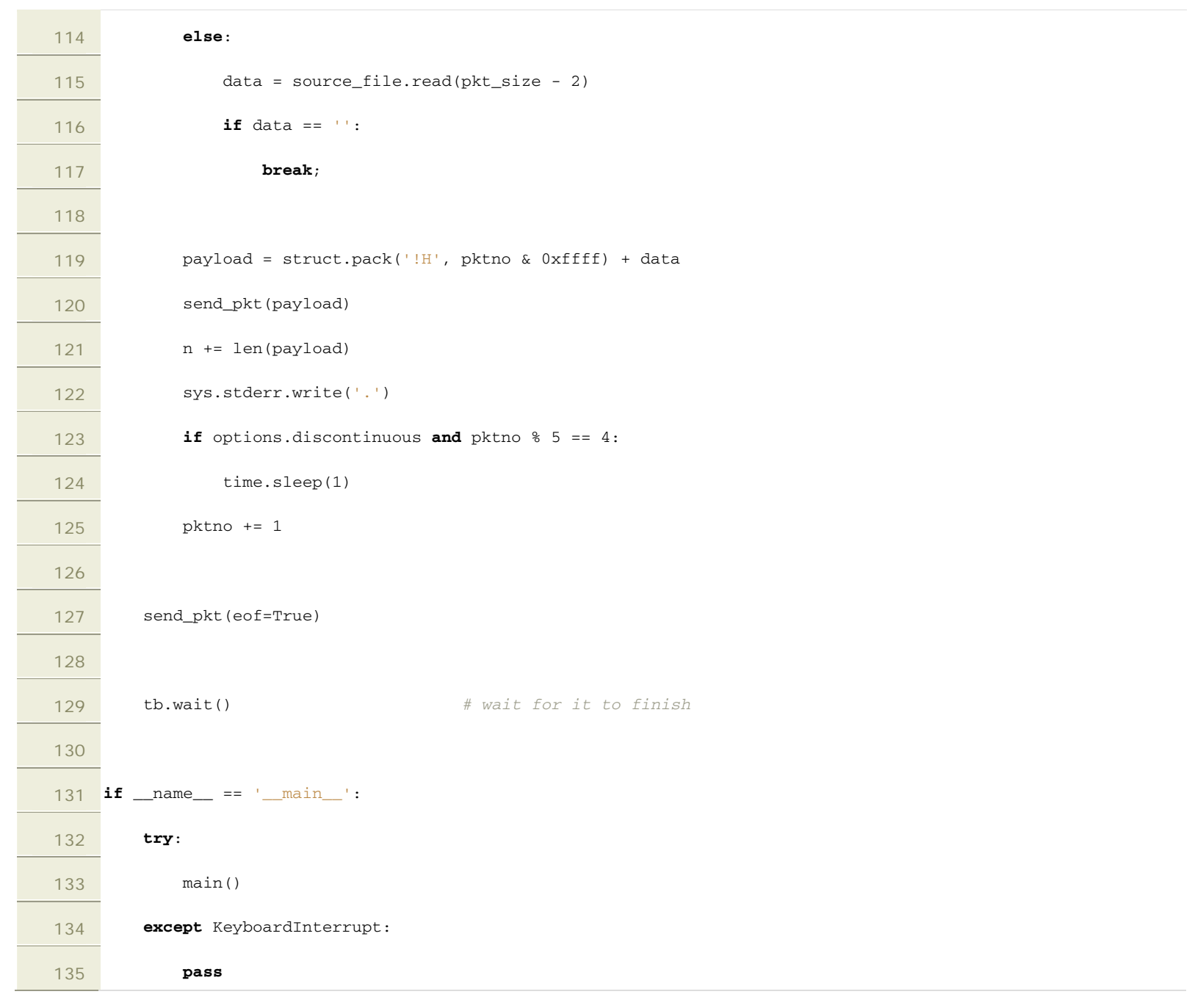




\section{Appendix B.2 benchmark_rx.py}

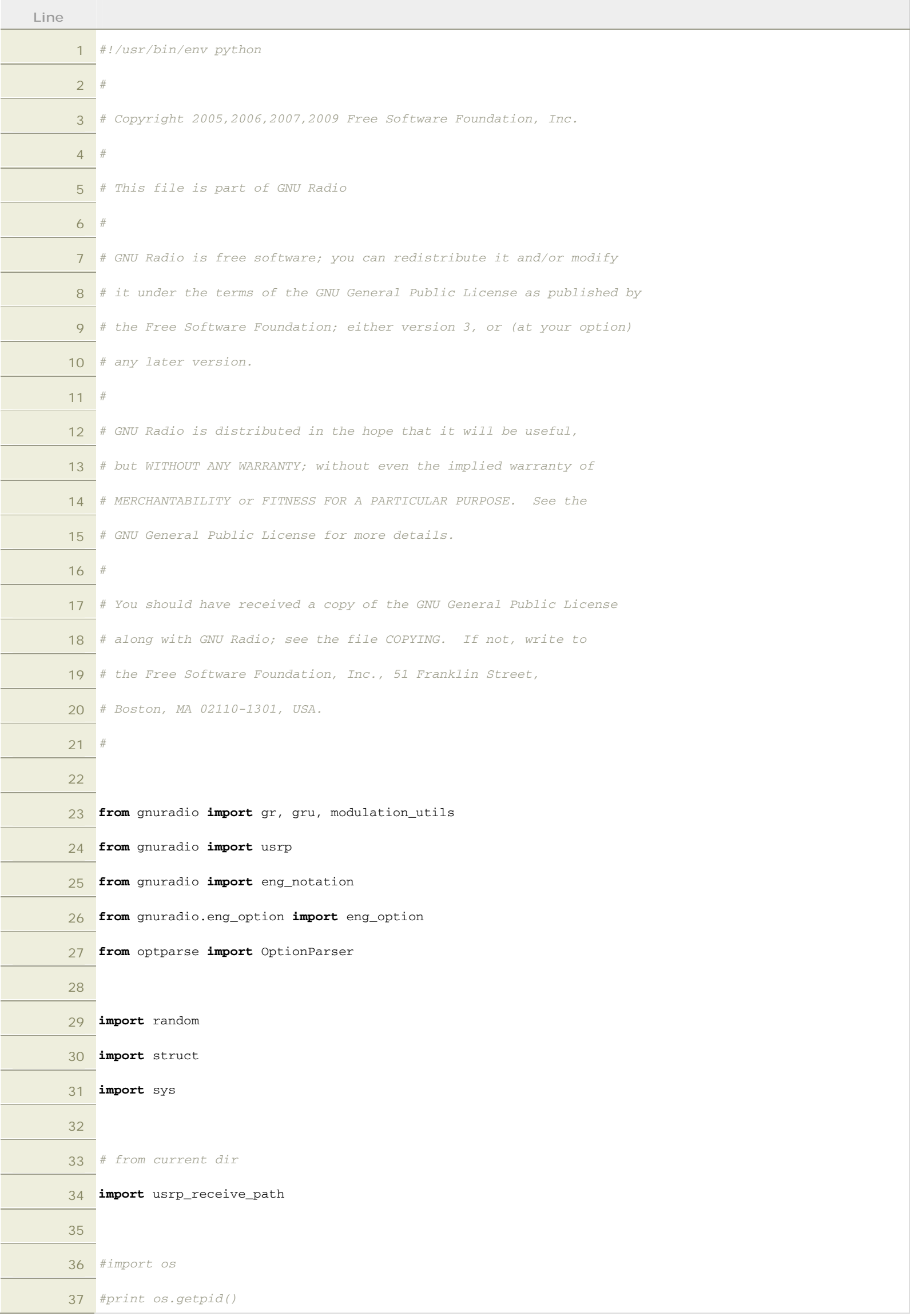




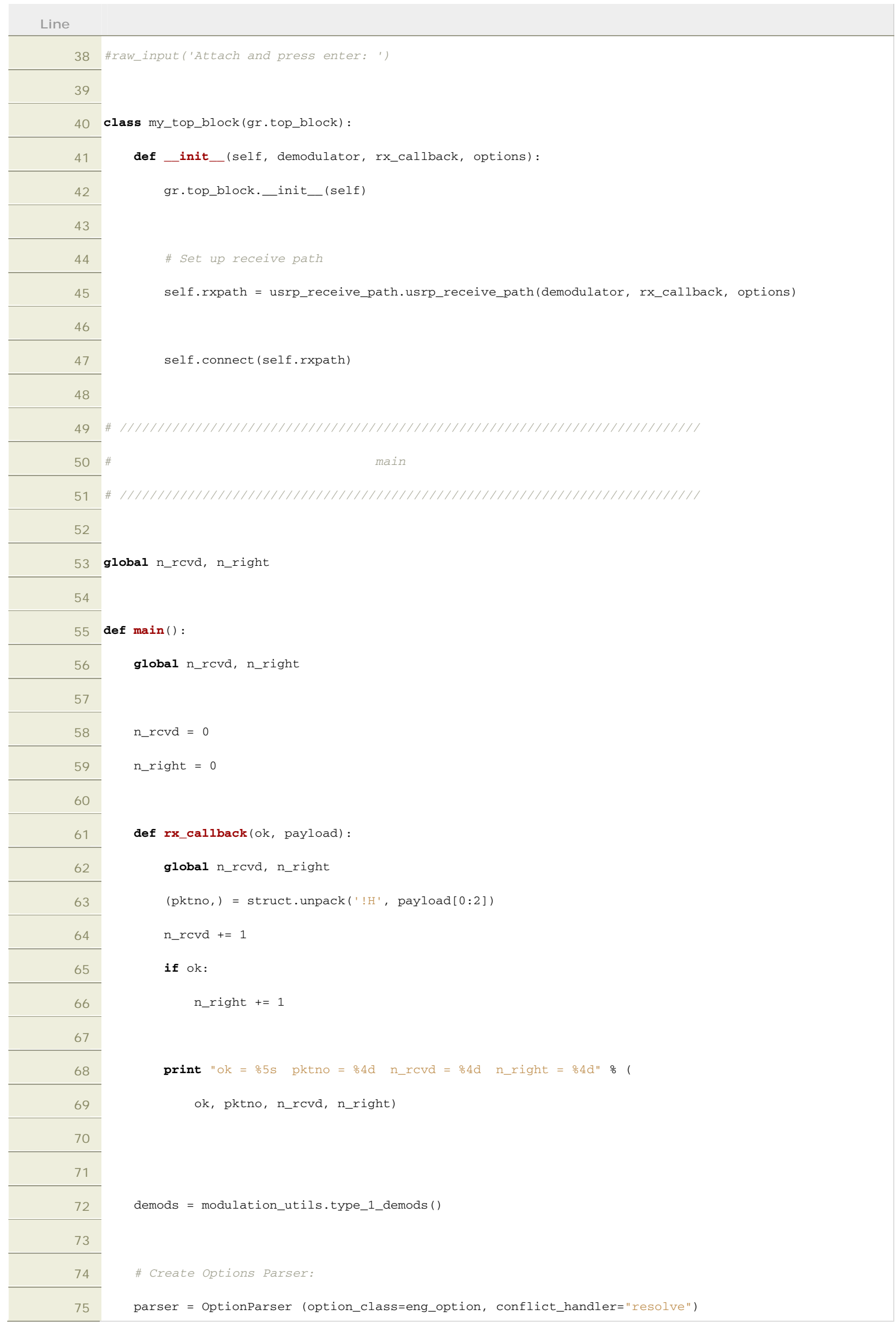




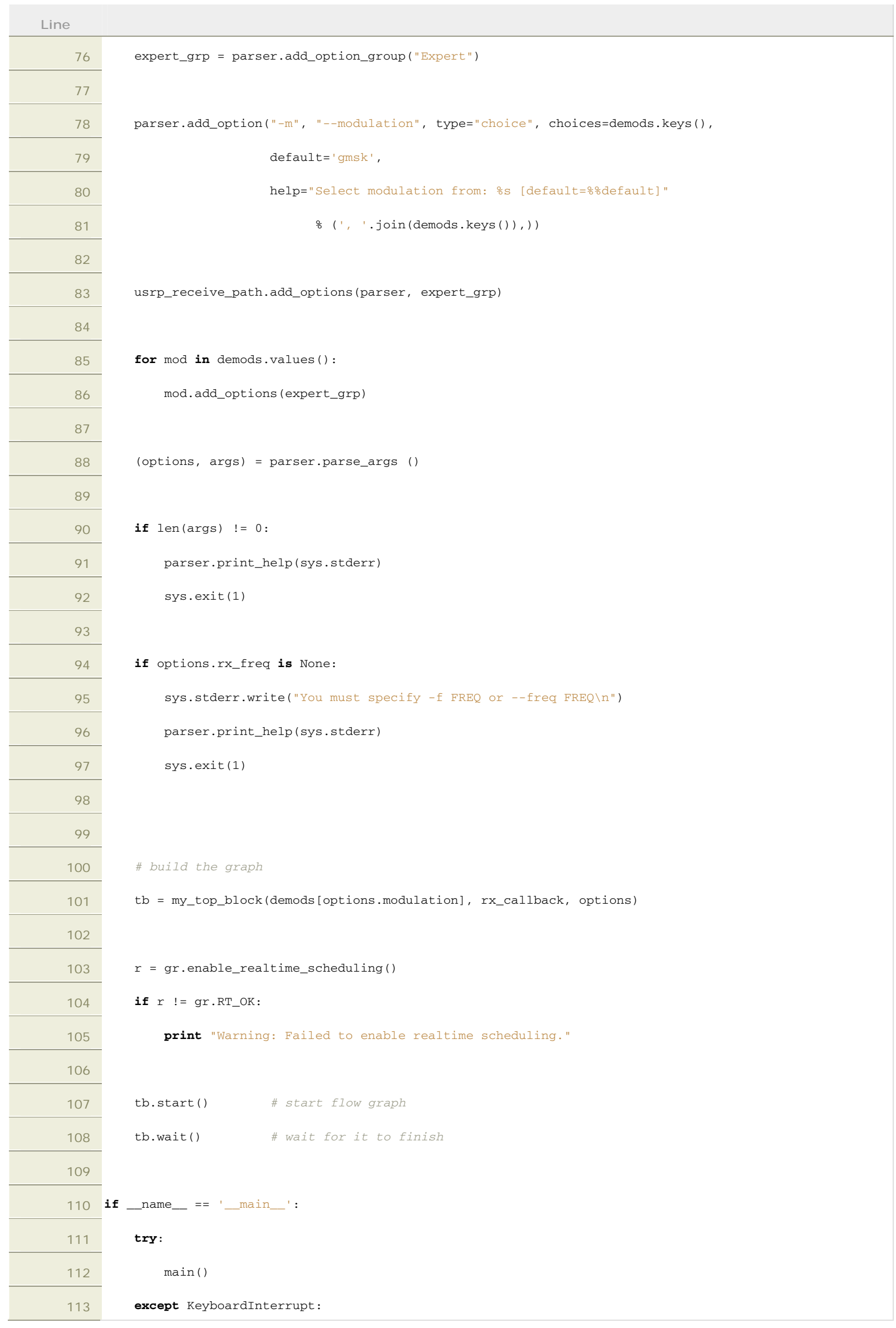




\section{Appendix B.3 tx_voice.py}

\begin{tabular}{|c|c|}
\hline 1 & \#!/usr/bin/env python \\
\hline 2 & \# \\
\hline 3 & \# Copyright 2005,2006,2007,2009 Free Software Foundation, Inc. \\
\hline 4 & \# \\
\hline 5 & \# This file is part of GNU Radio \\
\hline 6 & \# \\
\hline 7 & \# GNU Radio is free software; you can redistribute it and/or modify \\
\hline 8 & \# it under the terms of the GNU General Public License as published by \\
\hline 9 & \# the Free Software Foundation; either version 3, or (at your option) \\
\hline 10 & \# any later version. \\
\hline 11 & \# \\
\hline 12 & \# GNU Radio is distributed in the hope that it will be useful, \\
\hline 13 & \# but WITHOUT ANY WARRANTY; without even the implied warranty of \\
\hline 14 & \# MERCHANTABILITY OI FITNESS FOR A PARTICULAR PURPOSE. See the \\
\hline 15 & \# GNU General Public License for more details. \\
\hline 16 & \# \\
\hline 17 & \# You should have received a copy of the GNU General Public License \\
\hline 18 & \# along with GNU Radio; see the file COPYING. If not, write to \\
\hline 19 & \# the Free Software Foundation, Inc., 51 Franklin Street, \\
\hline 20 & \# Boston, MA 02110-1301, USA. \\
\hline 21 & \# \\
\hline 22 & \\
\hline 23 & from gnuradio import gr, gru, modulation_utils \\
\hline 24 & from gnuradio import usrp \\
\hline 25 & from gnuradio import audio \\
\hline 26 & from gnuradio import eng_notation \\
\hline 27 & from gnuradio.eng_option import eng_option \\
\hline 28 & from optparse import optionParser \\
\hline 29 & \\
\hline 30 & from gnuradio.vocoder import gsm_full_rate \\
\hline 31 & \\
\hline 32 & import random \\
\hline 33 & import time \\
\hline 34 & import struct \\
\hline 35 & import sys \\
\hline 36 & \\
\hline 37 & \# from current dir \\
\hline
\end{tabular}




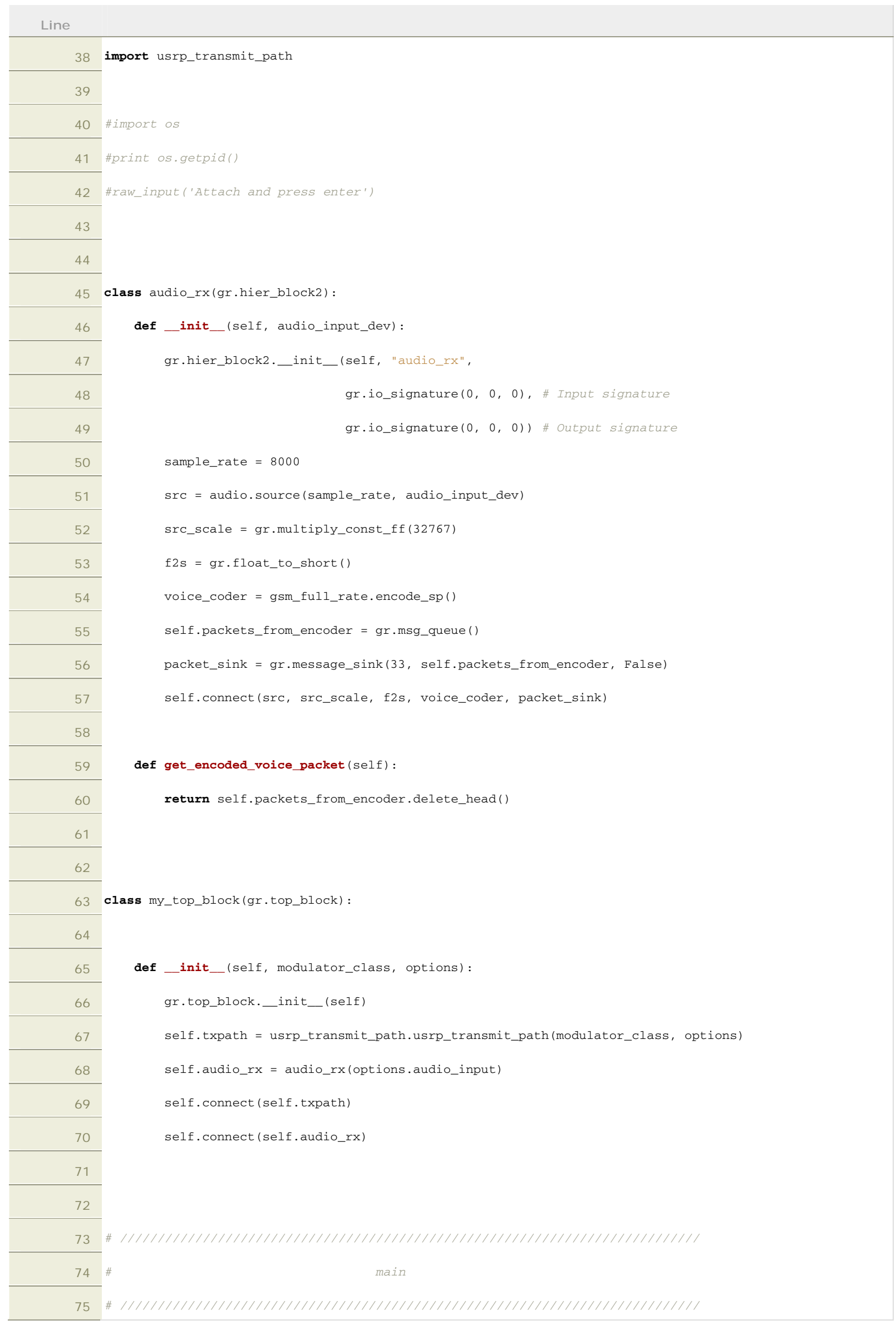




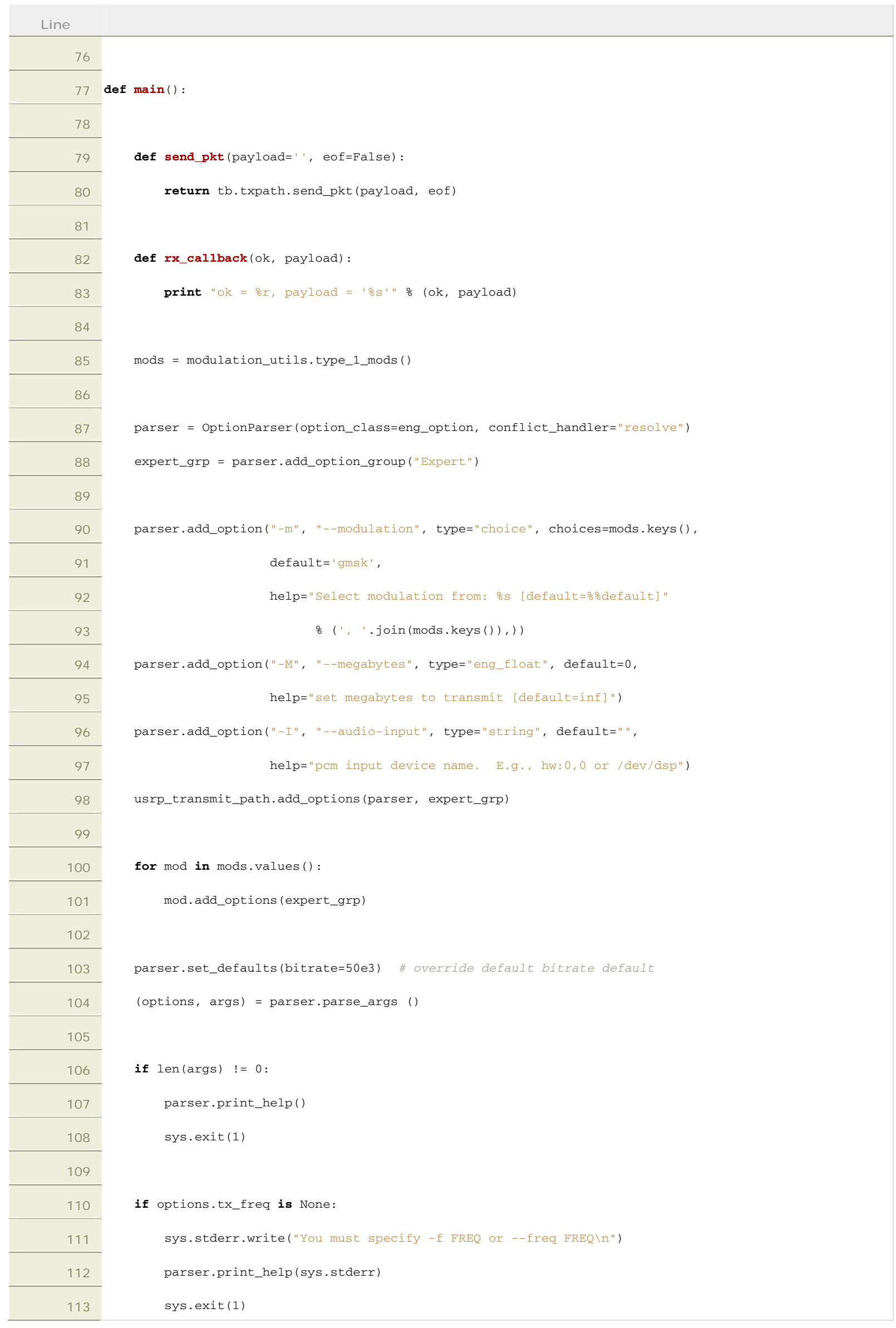




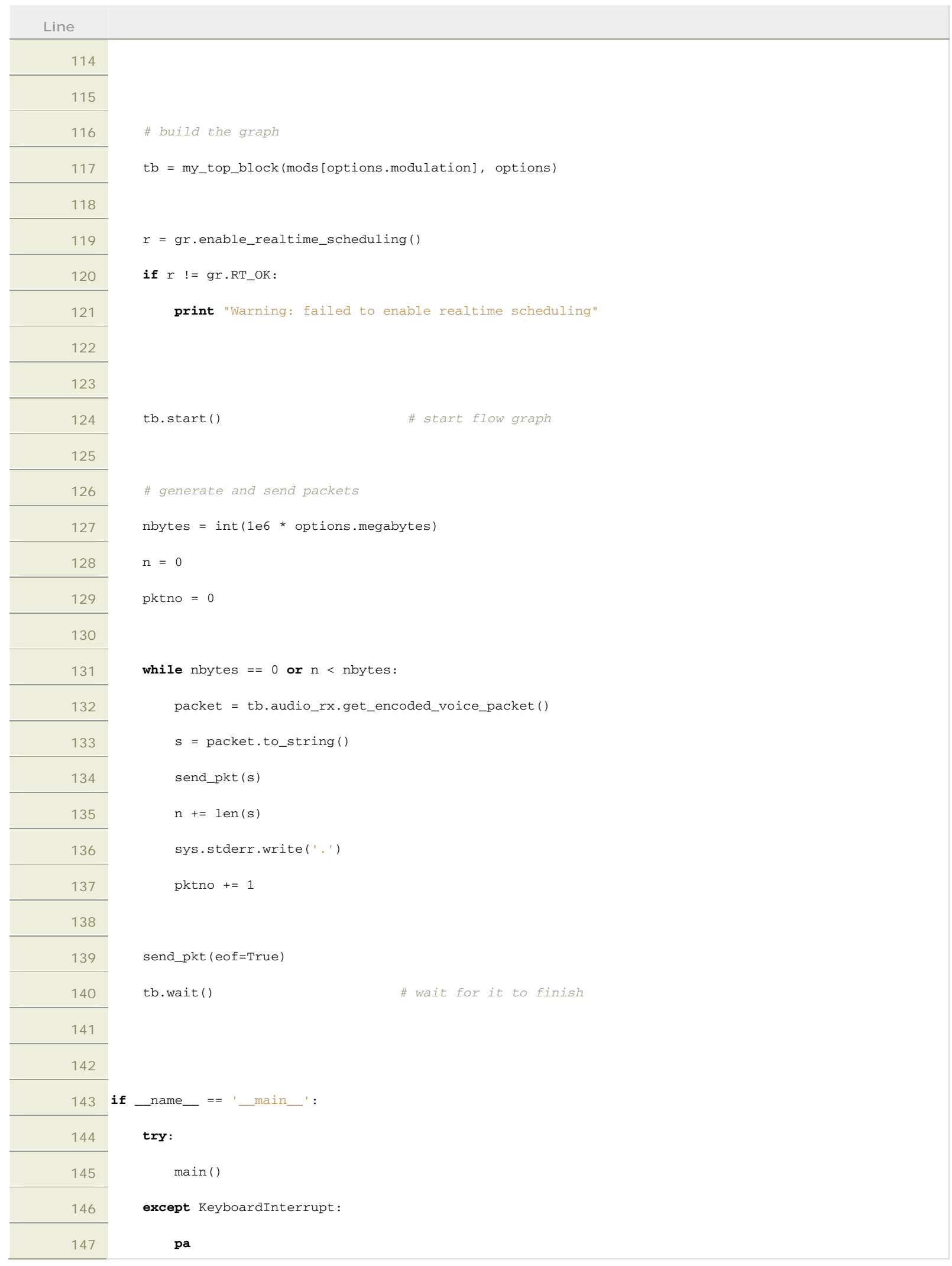




\section{Appendix B.4 rx_voice.py}

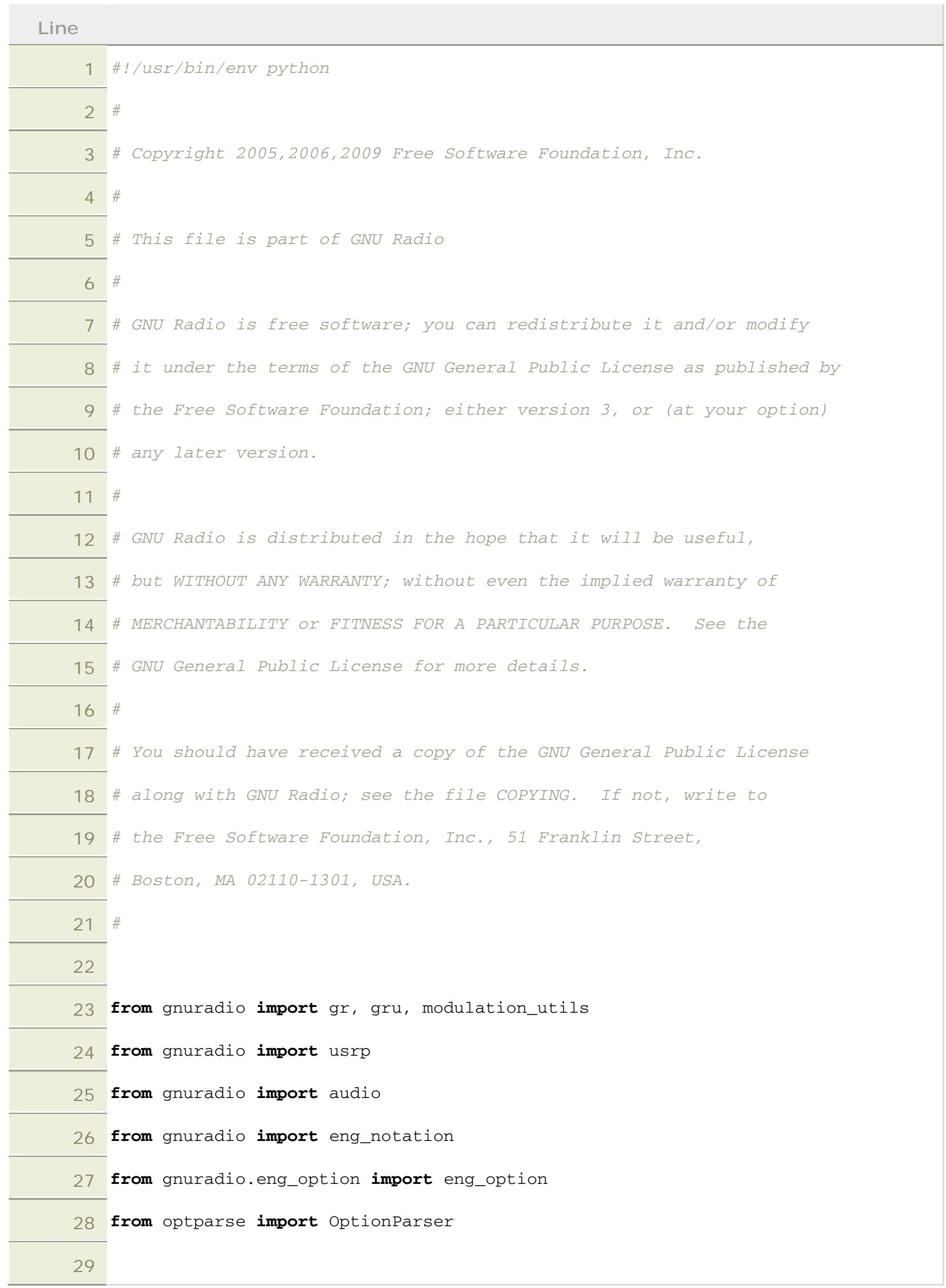




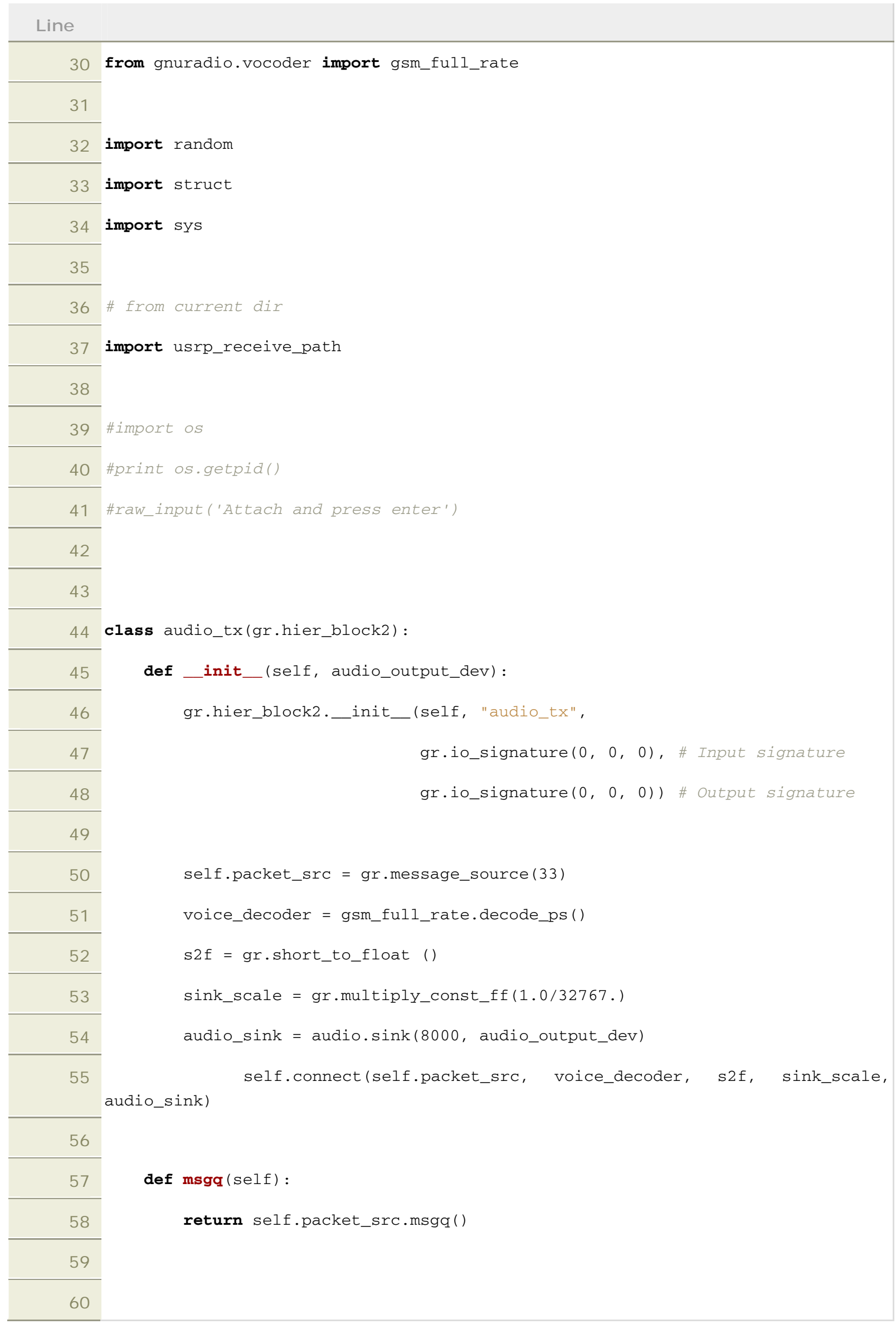




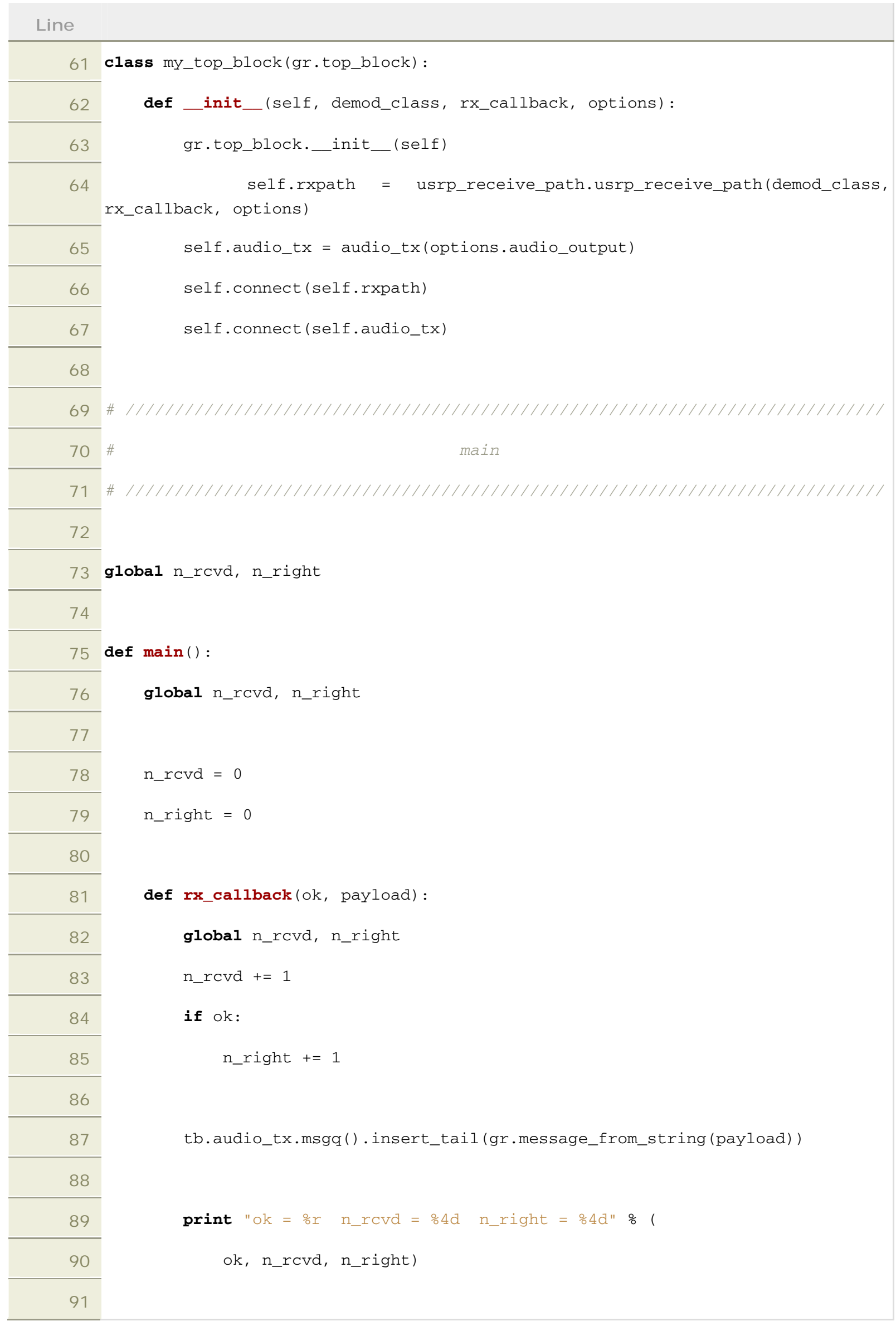




\begin{tabular}{|c|c|}
\hline Line & \\
\hline 92 & demods = modulation_utils.type_1_demods () \\
\hline 93 & \\
\hline 94 & \# Create Options Parser: \\
\hline 95 & parser = OptionParser (option_class=eng_option, conflict_handler="resolve") \\
\hline 96 & expert_grp = parser.add_option_group ("Expert") \\
\hline 97 & \\
\hline 98 & parser.add_option ("-m", " "--modulation", \\
\hline & choices=demods . keys ( ) \\
\hline 99 & default='gmsk', \\
\hline 100 & help="Select modulation from: \%s [default=\%\%default]" \\
\hline 101 & \% (', '.join (demods.keys ()), )) \\
\hline 102 & parser.add_option("-o", "--audio-output", type="string", default=" ", \\
\hline 103 & help="pcm output device name. E.g., hw:0,0 or /dev/dsp") \\
\hline 104 & usrp_receive_path.add_options (parser, expert_grp) \\
\hline 105 & \\
\hline 106 & for mod in demods.values(): \\
\hline 107 & mod.add_options (expert_grp) \\
\hline 108 & \\
\hline 109 & parser.set_defaults(bitrate=50e3) \# override default bitrate default \\
\hline 110 & (options, args) = parser.parse_args () \\
\hline 111 & \\
\hline 112 & if len(args) $\quad !=0:$ \\
\hline 113 & parser.print_help (sys.stderr) \\
\hline 114 & sys.exit (1) \\
\hline 115 & \\
\hline 116 & if options.rx_freq is None: \\
\hline 117 & sys.stderr.write("You must specify -f FREQ or --freq FREQ\n") \\
\hline 118 & parser.print_help (sys.stderr) \\
\hline 119 & sys.exit (1) \\
\hline 120 & \\
\hline 121 & \\
\hline 122 & \# build the graph \\
\hline
\end{tabular}




\begin{tabular}{|c|c|}
\hline Line & \\
\hline 123 & tb = my_top_block (demods[options.modulation], rx_callback, options) \\
\hline 124 & \\
\hline 125 & $r=g r . e n a b l e \_r e a l t i m e \_s c h e d u l i n g()$ \\
\hline 126 & if $r !=g r . R T \_O K:$ \\
\hline 127 & print "Warning: Failed to enable realtime scheduling." \\
\hline 128 & \\
\hline 129 & tb.run ( ) \\
\hline 130 & \\
\hline 131 & if __name__ $==$ '_main__ : \\
\hline 132 & try : \\
\hline 133 & $\operatorname{main}()$ \\
\hline 134 & except KeyboardInterrupt: \\
\hline 135 & pass \\
\hline
\end{tabular}




\section{Appendix B.5 tunnel.py}

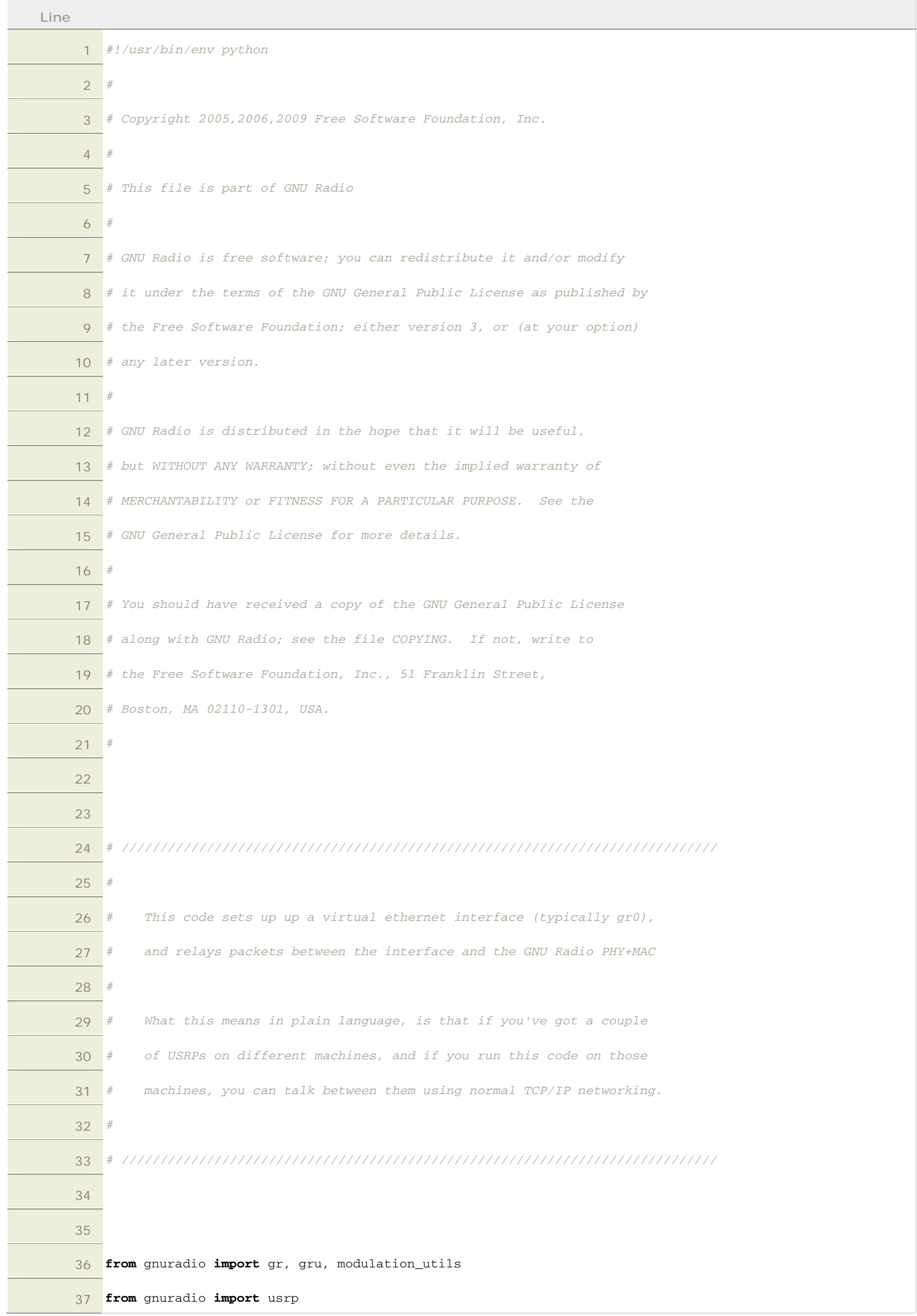




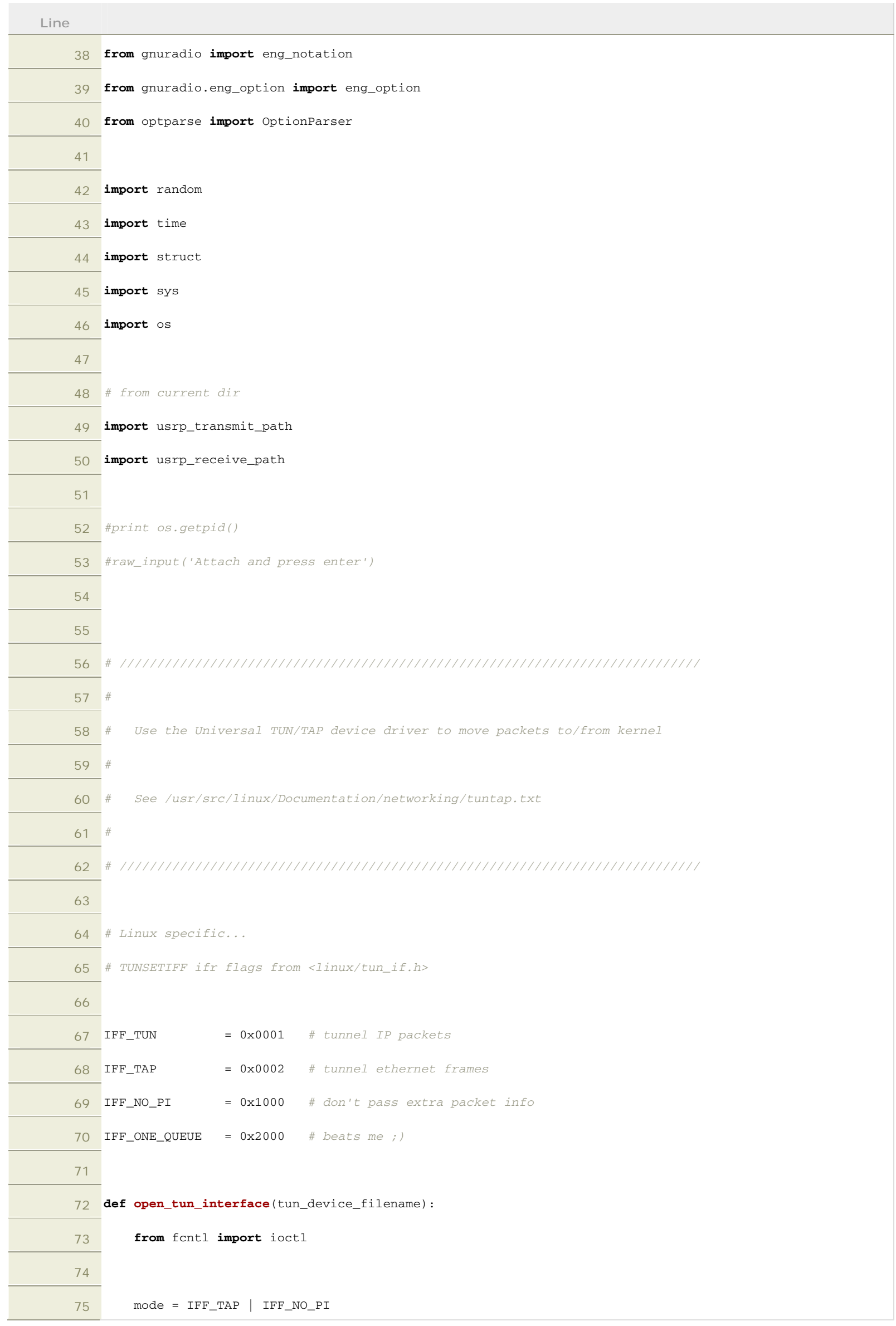




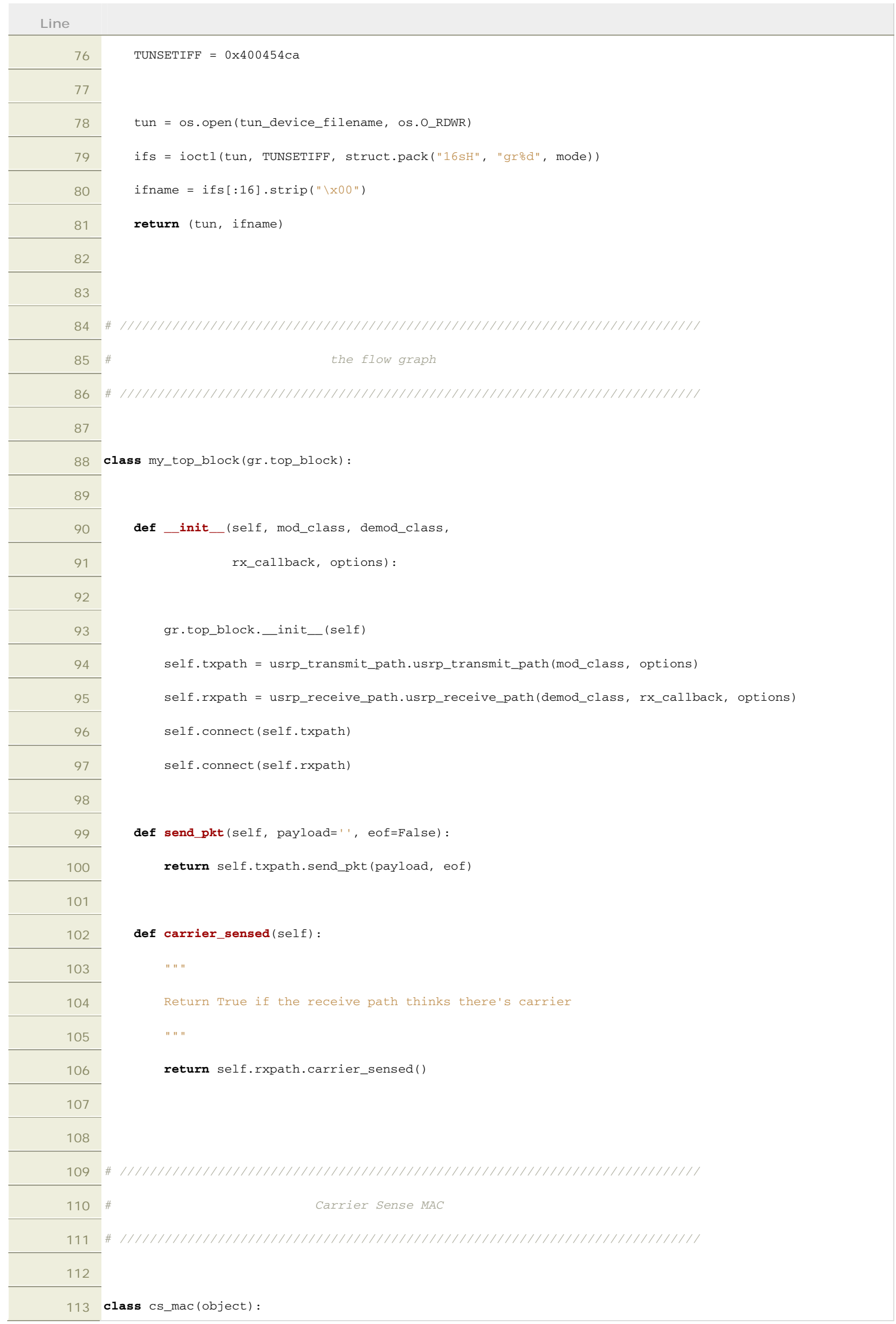




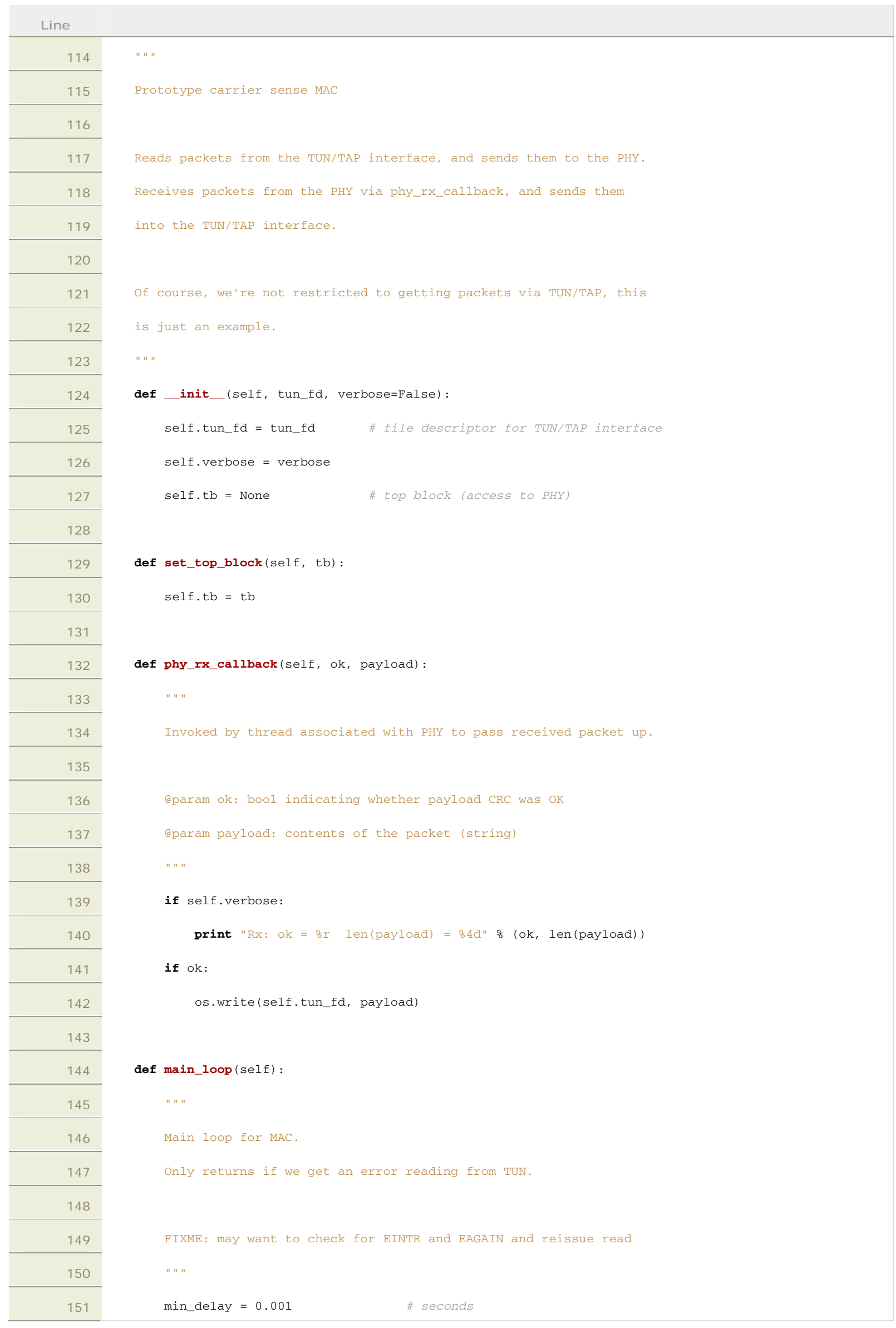




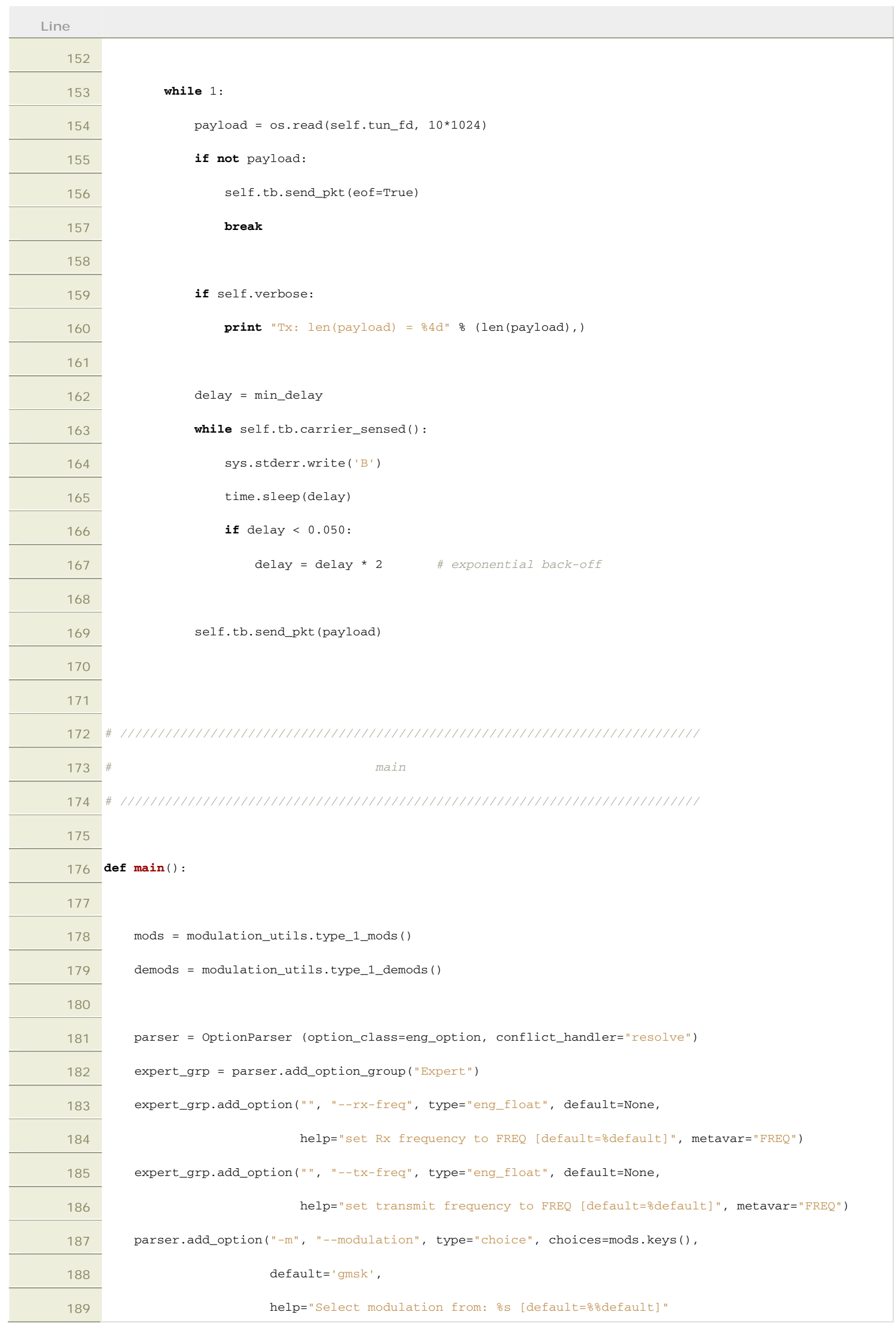




\begin{tabular}{|c|c|}
\hline Line & \\
\hline 190 & $\%(', \quad$ 'join (mods.keys ()$)))$, \\
\hline 191 & \\
\hline 192 & parser.add_option("-v","--verbose", action="store_true", default=False) \\
\hline 193 & expert_grp.add_option("-c", "--carrier-threshold", type="eng_float", default=30, \\
\hline 194 & help="set carrier detect threshold ( $d B$ ) [default=\%default]") \\
\hline 195 & expert_grp.add_option(" ","--tun-device-filename", default="/dev/net/tun", \\
\hline 196 & help="path to tun device file [default=\%default]") \\
\hline 197 & \\
\hline 198 & usrp_transmit_path.add_options (parser, expert_grp) \\
\hline 199 & usrp_receive_path.add_options(parser, expert_grp) \\
\hline 200 & \\
\hline 201 & for mod in mods.values (): \\
\hline 202 & mod.add_options (expert_grp) \\
\hline 203 & \\
\hline 204 & for demod in demods.values(): \\
\hline 205 & demod.add_options (expert_grp) \\
\hline 206 & \\
\hline 207 & (options, args) = parser.parse_args () \\
\hline 208 & if len(args) $!=0$ : \\
\hline 209 & parser.print_help (sys.stderr) \\
\hline 210 & sys.exit(1) \\
\hline 211 & \\
\hline 212 & \# open the TUN/TAP interface \\
\hline 213 & (tun_fd, tun_ifname) = open_tun_interface(options.tun_device_filename) \\
\hline 214 & \\
\hline 215 & \# Attempt to enable realtime scheduling \\
\hline 216 & $r=g r . e n a b l e \_r e a l t i m e \_s c h e d u l i n g()$ \\
\hline 217 & if $r==g r . R T \_O K:$ \\
\hline 218 & realtime $=$ True \\
\hline 219 & else: \\
\hline 220 & realtime $=$ False \\
\hline 221 & print "Note: failed to enable realtime scheduling" \\
\hline 222 & \\
\hline 223 & \\
\hline 224 & \# If the user hasn't set the fusb_* parameters on the command line, \\
\hline 225 & \# pick some values that will reduce latency. \\
\hline 226 & \\
\hline 227 & if options.fusb_block_size $==0$ and options.fusb_nblocks $==0$ : \\
\hline
\end{tabular}




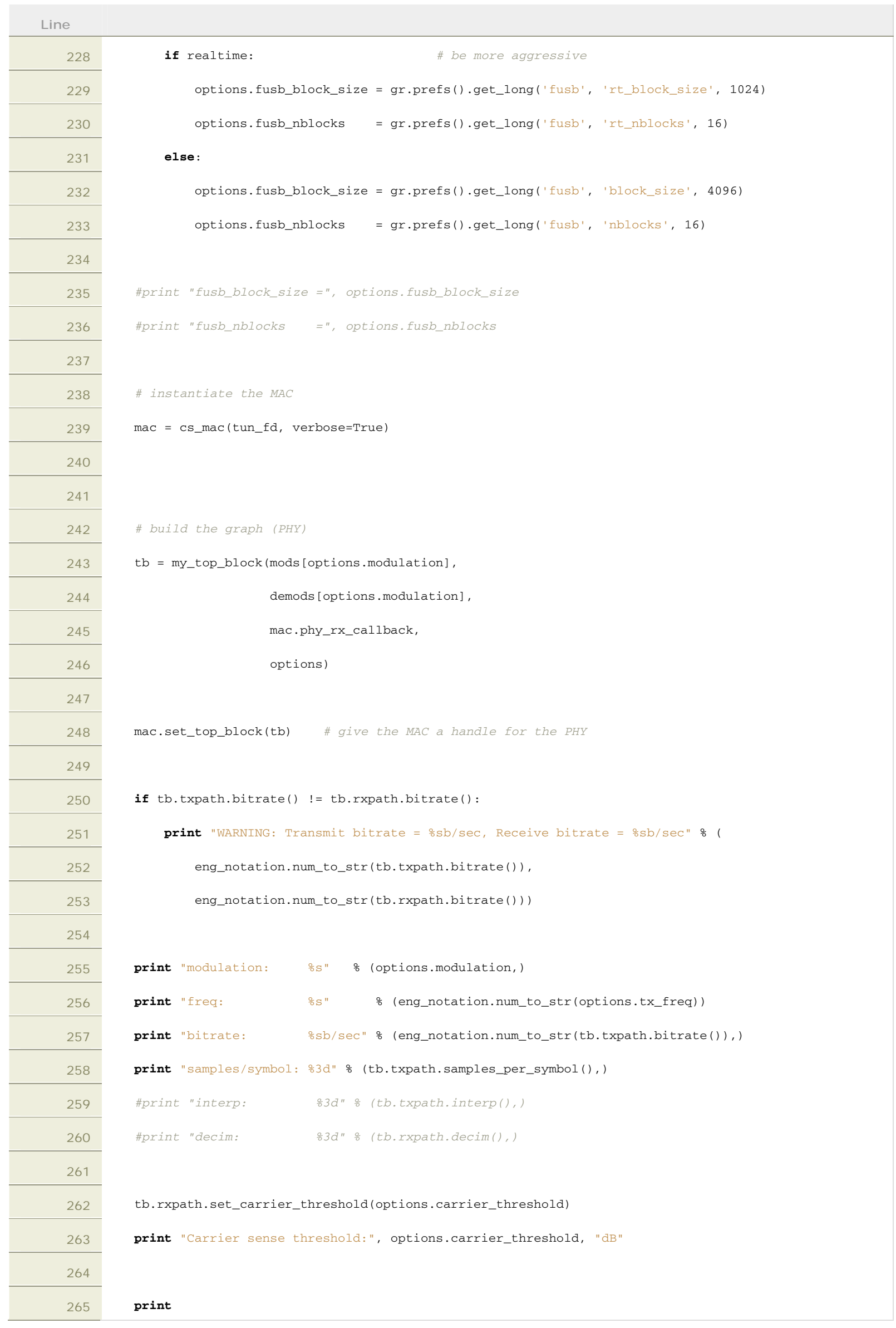




\begin{tabular}{|c|c|}
\hline Line & \\
\hline 266 & print "Allocated virtual ethernet interface: $\%$ s" \% (tun_ifname, ) \\
\hline 267 & print "You must now use ifconfig to set its IP address. E.g.," \\
\hline 268 & print \\
\hline 269 & print " \$ sudo ifconfig \%s 192.168.200.1" \% (tun_ifname, ) \\
\hline 270 & print \\
\hline 271 & print "Be sure to use a different address in the same subnet for each machine." \\
\hline 272 & print \\
\hline 273 & \\
\hline 274 & \\
\hline 275 & tb.start() \# Start executing the flow graph (runs in separate threads) \\
\hline 276 & \\
\hline 277 & mac.main_loop() \# don't expect this to return... \\
\hline 278 & \\
\hline 279 & tb.stop() \# but if it does, tell flow graph to stop. \\
\hline 280 & \# wait for it to finish \\
\hline 281 & \\
\hline 282 & \\
\hline 283 & if __name__ $==$ ' main_ \\
\hline 284 & try: \\
\hline 285 & $\operatorname{main}()$ \\
\hline 286 & except Keyboardinterrupt: \\
\hline 287 & pass \\
\hline
\end{tabular}


TRITA-ICT-EX-2009:206 